\title{
A novel family of fatty acyl thioesterases from
}

\section{Arabidopsis thaliana}

\author{
By: Christine Lowe
}

B.Sc. Honours, Carleton University, 2008.

A thesis submitted to the Faculty of Graduate Studies and Research in partial fulfillment of the requirements for the degree of

Master of Science

in

Biology

Carleton University

Ottawa ON, Canada

(C) 2010, Christine Lowe 


$\begin{array}{ll}\begin{array}{l}\text { Library and Archives } \\ \text { Canada }\end{array} & \begin{array}{l}\text { Bibliothèque et } \\ \text { Archives Canada }\end{array} \\ \begin{array}{l}\text { Published Heritage } \\ \text { Branch }\end{array} & \begin{array}{l}\text { Direction du } \\ \text { Patrimoine de lédition }\end{array} \\ \begin{array}{l}\text { 395 Wellington Street } \\ \text { Ottawa ON K1A ON4 } \\ \text { Canada }\end{array} & \begin{array}{l}\text { 395, rue Wellington } \\ \text { Ottawa ON K1A ON4 } \\ \text { Canada }\end{array}\end{array}$

Your file Votre référence

ISBN: 978-0-494-79608-5

Ourfile Notre référence

ISBN: 978-0-494-79608-5

NOTICE:

The author has granted a nonexclusive license allowing Library and Archives Canada to reproduce, publish, archive, preserve, conserve, communicate to the public by telecommunication or on the Internet, loan, distribute and sell theses worldwide, for commercial or noncommercial purposes, in microform, paper, electronic and/or any other formats.

The author retains copyright ownership and moral rights in this thesis. Neither the thesis nor substantial extracts from it may be printed or otherwise reproduced without the author's permission.
AVIS:

L'auteur a accordé une licence non exclusive permettant à la Bibliothèque et Archives Canada de reproduire, publier, archiver, sauvegarder, conserver, transmettre au public par télécommunication ou par l'Internet, prêter, distribuer et vendre des thèses partout dans le monde, à des fins commerciales ou autres, sur support microforme, papier, électronique et/ou autres formats.

L'auteur conserve la propriété du droit d'auteur et des droits moraux qui protège cette thèse. $\mathrm{Ni}$ la thèse ni des extraits substantiels de celle-ci ne doivent être imprimés ou autrement reproduits sans son autorisation.
In compliance with the Canadian Privacy Act some supporting forms may have been removed from this thesis.

While these forms may be included in the document page count, their removal does not represent any loss of content from the thesis.
Conformément à la loi canadienne sur la protection de la vie privée, quelques formulaires secondaires ont été enlevés de cette thèse.

Bien que ces formulaires aient inclus dans la pagination, il n'y aura aucun contenu manquant. 


\section{Abstract}

Extracellular lipid based barriers, such as the cuticle, suberin, and sporopollenin, protect plants from the environment. Despite the importance of these barriers, many aspects of extracellular lipid biosynthesis remain uncharacterized. I have identified a four member family of thioesterases from Arabidopsis thaliana termed MODIFIERS OF EXTRACELLULAR LIPIDS (MEL1-4). The gene expression patterns of three members of this family, $M E L 1, M E L 2$, and $M E L 4$, were found to correlate with the deposition of cuticle, suberin, and sporopollenin, respectively. Artificial microRNA silenced and overexpression lines were created to determine if MEL1 influences cuticle composition. The cuticular wax and cutin composition were analyzed, but no effect on the load or composition was apparent. This may be due to gene redundancy or insufficient silencing or over-expression of $M E L 1$. It is also possible that the effects on extracellular compounds are not detectable using common methods. MEL1 was shown in vitro to hydrolyze palmitoyl-CoA (16:0) into a free fatty acid and CoA. Members of the MEL family were also shown in $E$. coli to have acyl-ACP thioesterase activity toward $\beta$ ketoacyl-ACP and non-oxygenated acyl-ACP substrates. These results confirm that MEL proteins possess fatty acyl thioesterase activity and likely fulfill a role in extracellular lipid biosynthesis. I discuss several potential roles for thioesterases in extracellular lipid biosynthesis, such as in termination of fatty acid synthesis in the plastid, synthesis of $\beta$ ketoacids leading to the production of methylketones and/or alkanes, production of longchain or very-long-chain free fatty acids found in cutin and cuticular wax, respectively, and in regulation of extracellular lipid metabolism. The MEL enzymes are expected to fulfill one or more of these roles. 


\section{Acknowledgements}

I would like to thank my supervisor, Dr. Owen Rowland, for providing me with the opportunity to undertake this research project, and for his support and supervision during the last two years. I would also like to thank my M.Sc. graduate committee members, Dr. John Vierula and Dr. Gopal Subramaniam of Carleton University, for their suggestions through out the course of this work. I would like to express my appreciation to Dr. Ashkan Golshani for the use of his experimental equipment. I am also thankful for the support I have received from the members of the Rowland and Hepworth labs, and for their input and ideas that have proved invaluable throughout the course of this work. Finally, I am extremely grateful for the support I have received from my friends and family. 


\section{Statement of Contribution}

I would like to acknowledge the work of Dr. Owen Rowland, Swara Narayanan, Carlos Canez, Sollapura Vishwanath, and Xiaoxue Wen for their contributions to this thesis. I acknowledge Swara Narayanan for the creation of the promoter:GUS constructs for MEL1, MEL2, MEL3, and MEL4 and the transformation of Arabidopsis with these constructs, under my supervision. I also acknowledge Swara Narayanan, Carlos Canez, and Sollapura Vishwanath for analyzing and photographing the promoter:GUS transgenic plants. Dr. Owen Rowland and Xiaoxue Wen also contributed to this thesis by generating the constructs for the expression of MEL2 and MEL3 in E. coli. I would also like to express my appreciation to Dr. Frédéric Domergue (Centre National de la Recherché Scientifique, Laboratoire de Biogenèse Membranaire, Université Victor Ségalen Bordeaux, Bordeaux, France), for analyzing the cutin monomer composition, by GC-MS, of stem and leaf samples that I provided to him. 


\section{Table of Contents}

\section{Chapter1: Introduction}

1.1 Surface lipid barriers in plants...........................................

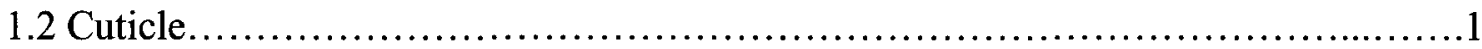

1.2.1 Cutin synthesis................................................

1.2.2 Cuticular wax synthesis......................................... 11

1.3 Roles for thioesterases in lipid biosynthesis.................................. 14

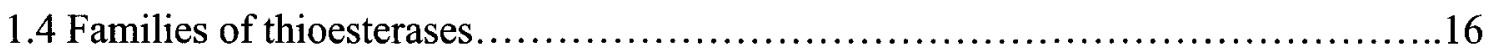

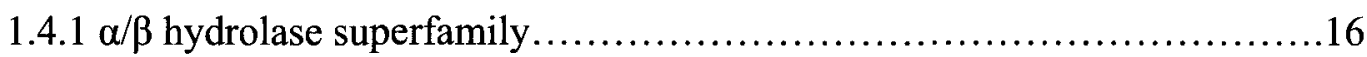

1.4.2 Hotdog fold superfamily ........................................20

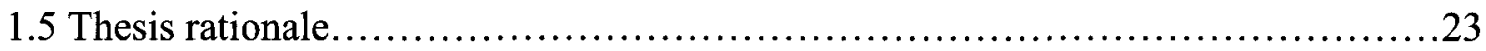

\section{Chapter 2: Materials and Methods}

2.1 Plant materials and growth conditions...................................... 24

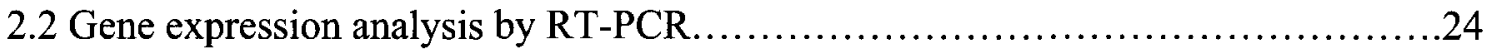

2.3 Cuticular wax analysis of Arabidopsis stems....................................25

2.4 Cutin analysis of Arabidopsis thaliana ......................................26

2.5 Ectopic expression of MEL1 in Arabidopsis................................27

2.6 Generation of artificial microRNA lines....................................29

2.7 In situ hybridization for the detection of $M E L 1$ transcript......................33

2.8 Promoter-glucuronidase (GUS) fusions and the GUS histochemical assay..........37

2.9 Plasmid constructs for the expression of MELs in $E$. coli..........................39

2.10 Media analysis........................................................40

2.11 Western blot analysis..................................................42 


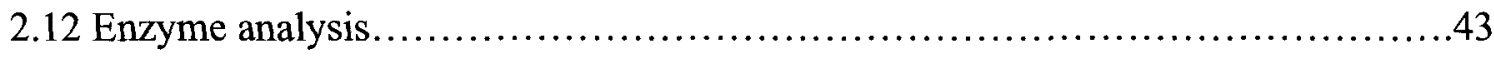

2.13 Co-regulation of extracellular lipid biosynthetic genes..........................46

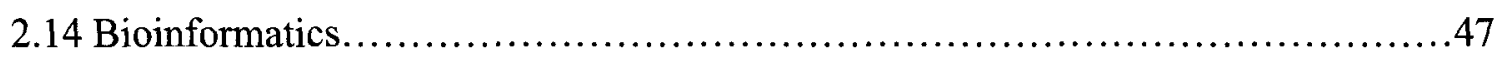

\section{Chapter 3: Results}

3.1 Identification of a thioesterase gene family associated with extracellular lipid

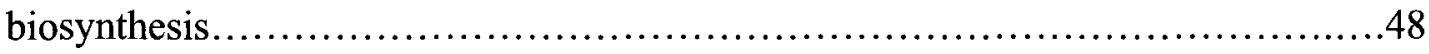

3.2 The predicted MEL proteins are Hotdog fold thioesterases.......................55

3.3 The $M E L$ family members have distinct gene expression patterns..................58

3.4 Silencing and over expression of MEL1 in Arabidopsis............................63

3.5 MEL1 has thioesterase activity in vitro ....................................... 74

3.6 The expression of MEL proteins in K27 E. coli cells leads to the production of novel

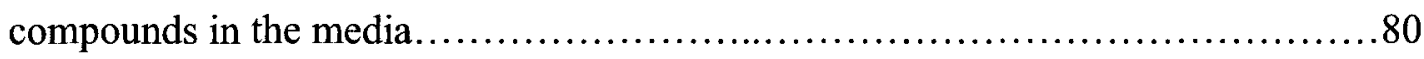

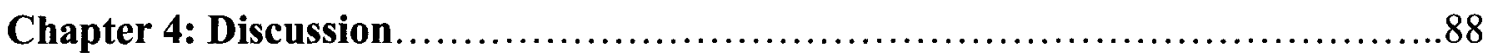

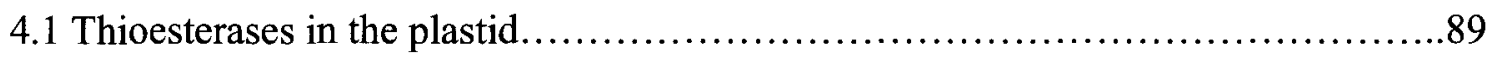

4.2 Thioesterases in the production of very-long-chain free fatty acids..................91

4.3 Thioesterases in the biosynthesis of $\beta$-ketoacids and alkanes....................92

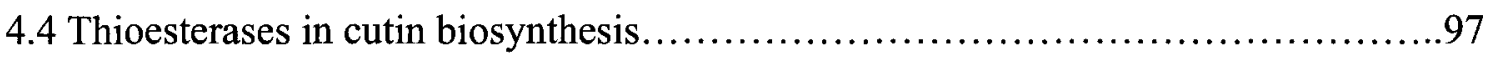

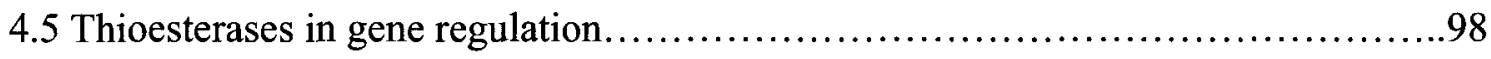

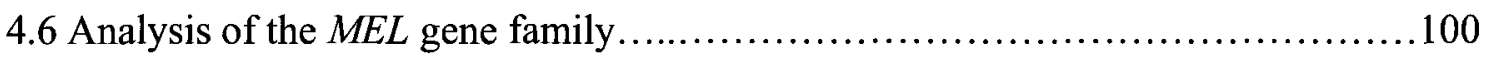

\section{Chapter 5: Conclusion and Future Research}

5.1 Future experiments to determine the physiological role of $M E L$ genes............102

5.2 Experiments to biochemically examine MEL enzymes...........................105

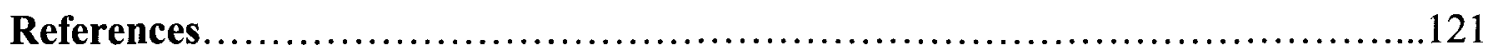




\section{List of Tables}

Table 1: PCR strategy to synthesize amiRNA sequences specific for $M E L 1 \ldots \ldots \ldots \ldots . . .31$

Table 2: Genes that are co-expressed with known cuticle biosynthetic genes in DNA microarray experiments...........................................50

Table 3: MEL1 co-expresses with known cuticle biosynthesis genes.................52

Table 4: Genes that are co-expressed with known suberin biosynthetic genes in DNA microarray experiments............................................53

Table 5: MEL2 co-expresses with known suberin biosynthesis genes...................54 


\section{List of Figures}

Figure 1: Schematic representation of the cuticle................................

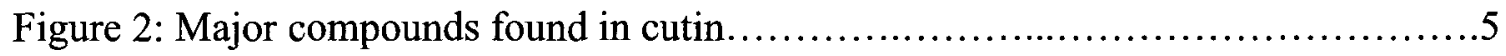

Figure 3: Major compounds found in the stem cuticular wax of Arabidopsis.............6

Figure 4: A model for cutin biosynthesis................................... 10

Figure 5: A simplified pathway for wax biosynthesis in Arabidopsis...................13

Figure 6: The fatty acyl thioesterase reaction.................................. 15

Figure 7: The $\alpha / \beta$ hydrolase fold is one of two major superfamilies with thioesterase

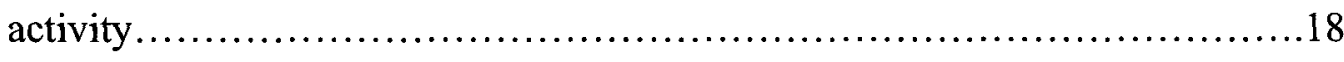

Figure 8: Conformations of the Hotdog fold superfamily..........................22

Figure 9: A model for artificial amiRNA synthesis and how amiRNAs silence genes of interest............................................................ 32

Figure 10: The MEL protein family shares a high level of similarity and contains a single characteristic Hotdog fold domain....................................56

Figure 11: RT-PCR for the tissue specific expression of the $M E L$ gene family..........60

Figure 12: Expression of the GUS reporter gene driven by the $M E L$ promoters..........61

Figure 13: MEL1 epidermal-specific expression as determined by in situ hybridization..62 Figure 14: The MEL1_amiRNA-A and MEL1_amiRNA-B sequences aligned with the coding sequence from the Arabidopsis MEL genes......................66

Figure 15: AmiRNA lines were developed to decrease $M E L 1$ transcript levels..........68

Figure 16: Altering the transcript level of $M E L I$ does not appear to affect cuticular wax composition

Figure 17: MEL1 amiRNA lines used for cutin composition analysis. 
Figure 18: The cutin composition of leaf and stem samples from MEL1 amiRNA-1 and MEL1 amiRNA-4 lines.................................................

Figure 19: The MEL proteins expressed in BL21(DE3)pLysS E. coli cells..............76

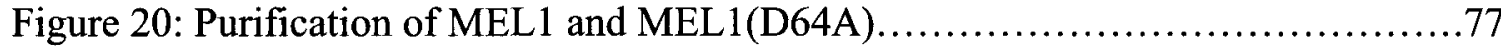

Figure 21: MEL1 is most active in sodium phosphate buffer.........................78

Figure 22: MEL1 is an active thioesterase using palmitoyl-CoA as a substrate...........79

Figure 23: Expression of MEL1, MEL2, MEL3, MEL1(D64A), and FATB in the K27(DE3) (fadD) E. coli cell line .83

Figure 24: Expression of MEL proteins in the K27 ( $f a d D)$ strain of $E$. coli leads to the accumulation of novel fatty acids in the media .84

Figure 25: The expression of MEL proteins in the K27 strain of E. coli leads to the accumulation of novel $\beta$-ketoacids in the media .86

Figure 26: Two models for alkane biosynthesis in plants .96 


\section{List of appendices}

Appendix 1: Primer list.................................................... 108

Appendix 2: Vector maps of constructs.................................... 110

Appendix 3: Previously identified cuticle associated genes.......................113

Appendix 4: Previously identified suberin associated genes.......................115

Appendix 5: Amino acid sequence alignment of the predicted MEL proteins with related single-domain Hotdog fold proteins...............................116

Appendix 6: Cuticular aliphatic compounds in the first and second generations of $M E L 1$

amiRNA and $M E L 1-35 S$ transgenic lines............................ 120 


\section{List of Abbreviations}

1-OH: primary alcohol

2-OH: secondary alcohol

2OH: 2-hydroxy fatty acid

4HBT: 4-hydroxybenzoyl-CoA thioesterase

ACP: acyl carrier protein

ALD: aldehyde

ALK: alkane

amiRNA: artificial microRNA

ATT1: aberrant induction of type three genes

BSTFA+TMCS: bis(trimethylsilyl)trifluoroacetamide:trimethylchlorosilane

CER: eceriferum

CoA: coenzyme A

Col-WT: Columbia-0 wild-type Arabidopsis thaliana

DCA: dicarboxylic acid

DEPC: diethylpyrocarbonate

diOH: 9,10 dihydroxy fatty acid

DTNB: 5,5'-dithiobis-(2-nitrobenzoic acid)

DW: dry weight

ER: endoplasmic reticulum

FAE: fatty acid elongase

FAR: fatty acyl-CoA reductase

FAS: fatty acid synthase 
FAT: fatty acyl-ACP thioesterase

FFA: free fatty acid

FFA: free fatty acids

GAPC: glyceraldehyde -3 - phosphate dehydrogenase

GC-FID: gas chromatography-flame ionization detector

GC-MS: gas chromatography-mass spectrophotometry

GPAT: glycerol-3-phosphate acyltransferase

GUS: glucuronidase

HNF: human nuclear factor

IPTG: isopropyl $\beta$-D-1-thiogalactopyranoside

KET: ketone

LACS: long-chain acyl-CoA synthetase

LCR: lacerata

Lf: rosette leaf

MAH: mid-chain alkane hydroxylase

MEL: modifier of extracellular lipids

MKS: methylketone synthase

MS: male sterility

$\mathrm{OH}$ : alcohol

PBS: phosphate buffered saline

PPAR: peroxisome proliferator-activated receptor

RT-PCR: reverse transcription PCR

Rt: roots 
St: stem

TBS-T: TRIS buffered saline with tween

TNB: 5-thio-2-nitrobenzoate

VLCFA: very-long-chain fatty acids

wOH: $\omega$-hydroxy fatty acid

WSD: wax synthase/diacylglycerol acyltransferase 


\section{Chapter 1: Introduction}

1.1 Surface lipid barriers in plants

Plants have three major extracellular lipid barriers for protection against various biotic and abiotic stresses: (1) the cuticle, which coats aerial surfaces, (2) suberin, found associated with the cell wall of various external and internal tissue layers including the root endodermis and peridermis, and (3) sporopollenin, found in the outer wall of pollen. The cuticle functions to protect plants from stresses such as UV radiation and nonstomatal water loss (Krauss et al., 1997; Riederer and Schreiber, 2001). The cuticle also functions to mediate plant-insect interactions and to inhibit infection by some pathogens (Kolattukudy, 1987; Jenks et al, 1994; Markstädter et al., 2000). In addition, the cuticle influences plant development by preventing organs from fusing (Sieber, et al., 2000). Suberin functions primarily to prevent uncontrolled water transport and to limit the uptake of solutes from the soil (Franke and Schreiber, 2007; Bernards, 2002). Sporopollenin makes up the protective exine layer that protects pollen grains from desiccation and makes them extremely resistant to degradation (Piffanelli et al., 1998). While the synthesis of each of these polymers begins with the de novo synthesis of fatty acids in the plastid, specific modifications occur in the endoplasmic reticulum (ER). The various lipids are then secreted to the outside of the cell.

\subsection{Cuticle}

The cuticle is a highly hydrophobic barrier synthesized and secreted by the epidermal cells of vascular plants. The mature cuticle is composed of two distinct components: (1) a lipid-based cutin layer embedded with various waxes, and (2) an outer 
layer composed primarily of wax (Figure 1). Cutin is a polyester matrix containing glycerol and esterified hydroxylated and dicarboxylic acids fatty acids of 16 or 18 carbons in length (Figure 2). Cutin also contains other minor components such as unsubstituted fatty acids and primary alcohols. This layer is highly resistant to mechanical damage and provides structure to the cuticle (Heredia, 2003). Cuticular wax is typically comprised of very-long-chain fatty acids (VLCFA) consisting of 20 to 32 carbons and a variety of derivatives including alkanes, primary and secondary alcohols, ketones, aldehydes, and wax esters (Figure 3) (Samuels, et al. 2008). The specific chemical make up is dependent on the organ and plant type. For example, while all of the above described compounds are found in Arabidopsis stem cuticle, the leaf cuticle lacks secondary alcohols and ketones (Jenks et al., 1995). In addition to forming epicuticular wax crystals, waxes are also embedded within the cutin matrix.

The synthesis of all lipid polymers begins with the de novo biosynthesis of acylacyl carrier protein (ACP) chains within the plastid by the fatty acid synthase (FAS) complex. This process begins with a condensation reaction between acetyl-coenzyme A (CoA) and malonyl-ACP. Following this condensation, the fatty acyl chain undergoes two separate reduction reactions and a dehydration step, resulting in a fatty acyl chain that is two carbons longer. Subsequent rounds of $\mathrm{C} 2$ additions from malonyl-ACP yield fatty acyl chains that are C16 and C18 in length (Ohlrogge and Jaworski, 1997). Once these molecules reach either $\mathrm{C} 16$ or $\mathrm{C} 18$ in length, the fatty acids are hydrolyzed from the ACP by acyl-ACP thioesterases (e.g. FATB and FATA) and exported from the plastid (Bonaventure et al. 2003; Salas and Ohlrogge, 2002). Free fatty acids at the outer envelope of the plastid are activated through the addition of CoA by a long-chain acyl- 
CoA synthase (LACS) (Schnurr, et al., 2002). A portion of the C16/C18 fatty acyl pool is used for plastidial membrane lipid synthesis within the plastid, while the majority is relocated to the ER for modifications (e.g. desaturation, oxidation) to synthesize the cutin and cuticular waxes that make up the cuticle, as well as other membrane and storage lipids (Ohlrogge and Jaworski, 1997). Many enzymes involved in the biosynthesis of the cuticle have been investigated and identified (Appendix 3). In spite of recent progress, several aspects of the cuticle biosynthetic pathways remain unknown. 


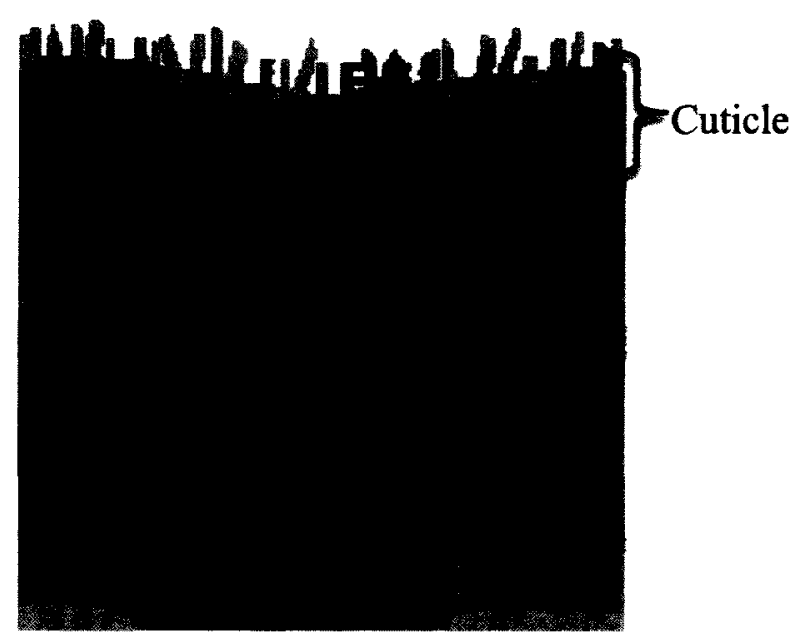

Figure 1: Schematic representation of the cuticle.

The cuticle is deposited outside of the plant cell wall (PW). This representation of a leaf cross-section illustrates the two distinct layers of the cuticle proper, the cutin layer (C), and the epicuticular wax layer (EW). This model also shows the cuticular layer (CL), which is composed of cutin and polysaccharides from the cell wall. Adapted from Pollard et al., 2008. 
A)<smiles>O=C(O)CCCCCCCCCCCCCCCCO</smiles>

B)<smiles>O=C(O)CCCCCCCCCCCCCCC(=O)O</smiles><smiles>O=C(O)CCCCCCCCC(O)CCCCCO</smiles><smiles>O=C(O)CCCCCCCCC(O)CCCCCCC(=O)O</smiles>

Figure 2: Major compounds found in the cutin matrix.

The Arabidopsis cutin matrix is primarily made up of hydroxylated (A), $\alpha, \omega$-dicarboxylic acids (B), mid-chain hydroxylated fatty acids (C), and mid-chain hydroxylated dicarboxylic acids (D). 


Fatty acid

Figure 3: Major compounds found in the stem cuticular wax of Arabidopsis.

The cuticular waxes of Arabidopsis are made up of various aliphatic compounds such as fatty acids, primary alcohols, wax esters, aldehydes, alkanes, secondary alcohols, and ketones. These compounds are typically found in other plant cuticles, but in varying proportions. 
1.2.1 Cutin synthesis

Cutin accounts for a large portion of the cuticle ( 40 to $80 \%$ depending on the plant and tissue type) and is primarily composed of hydroxylated C16 and C18 fatty acids and glycerol (Heredia, 2003; Pollard et al., 2008). Detailed analysis of the cutin component of the cuticle has thus far been challenging due to its insolubility in organic solvents. Cutin must be chemically depolymerized before analysis and therefore only the levels of the monomeric building blocks can be detected (Bonaventure et al., 2004, Kolattukudy, 2001). To build cutin, the fatty acid carboxyl groups esterify with glycerol as well as with the mid-chain hydroxyl groups from the various fatty acids to generate a three-dimensional matrix (Ray et al., 1998a, b; Graça and Lamosa, 2010).

The enzymes involved in cutin synthesis have only recently begun to be identified and characterized, largely in Arabidopsis (Appendix 3). LACS1 and LACS2 are long chain acyl-CoA synthetases required for cutin synthesis. Plants lacking LACS2 transcript have a thinner cuticle layer than wild-type and exhibit typical features of cutin defects, such as increased chlorophyll leaching (Schnurr et al., 2004). LACS1 mutants have a less dramatic cutin defect, but are deficient in C16 monomer derivatives (Lü et al., 2009). The double lacs 1 lacs 2 mutant has a major cutin defect, showing a dramatic increase in non-stomatal water loss (Lü et al., 2009; Weng et al., 2010). Two hydroxylases, LCR and ATT1, have been implicated in cutin biosynthesis (Xiao et al., 2004; Wellesen et al., 2001). Both of these enzymes are cytochrome P450 monooxygenases from the CYP86 family of cytochromes. Plants with mutations in these genes show characteristics of cutin defects, such as increased susceptibility to disease in the case of attl and organ fusions in the case of $l$ cr (Xiao et al., 2004; Wellesen et al., 2001). Both of these genes are thought 
to hydroxylate free fatty acids into $\omega$-hydroxy fatty acid or $\alpha, \omega$-dihydroxycarboxylic acid cutin monomers. It is known that hydroxylated fatty acids in cutin are esterified to glycerol, an activity that can be attributed to a glycerol-3-phosphate acyltransferase (GPAT), which joins hydroxy fatty acyl-CoAs to glycerol to form mono and diacylglycerolipids in cutin. This activity has been attributed to GPAT4 and GPAT8. These genes act redundantly and the gpat 4 gpat 8 double mutant displays major physiological changes indicative of cuticular defects, such as increased non-stomatal water loss (Li et al., 2007). GPAT4 and GPAT8 are not the only acyltransferase enzymes responsible for the polymerization of the cutin compounds. Recently a cytosolic acyltransferase, DCR, has been identified. DCR plays a role in the incorporation of 9,10,16-hydroxy-hexadecanedioic acid into the cutin matrix as the $d c r$ mutant has a marked decrease in this compound following cutin depolymerization (Panikashvili et al., 2009). However, the specific role of this acyltransferase in the overall pathway is currently unknown.

A mystery in cutin monomer biosynthesis is the order in which these events occur. One model has a thioesterase releasing a free fatty acid, which is subsequently oxidized to a hydroxy fatty acid, which then is reactivated to a hydroxy fatty acyl-CoA before being transferred to glycerol (Figure 4A). The hydroxylases ATT1 and LCR from Arabidopsis show activity toward free fatty acids in vitro, while ATT1 does not appear to have any activity toward the activated acyl-CoA (Benveniste et al., 2006; Wellesen et al., 2001). In Petunia hybrida, however, a fatty acyl-CoA $\omega$-hydroxylase has been identified that preferentially hydroxylates fatty acyl-CoA substrates over free fatty acids (Han et al., 2010). Nevertheless, the current evidence in Arabidopsis indicates that the activated 
acyl-CoA substrates imported into the ER are likely converted to a free fatty acid prior to hydroxylation. These free hydroxy-fatty acids would need to be reactivated by a LACS, such as LACS1 or LACS2, for the GPAT to form the mono and diacylglycerolipids (Lü et al., 2009). It has been reported that LACS2 has a higher in vitro activity with hydroxylated fatty acids over non-hydroxylated fatty acids (Schnurr et al., 2004). No enzyme has yet to be identified fulfilling the role of an acyl-CoA thioesterase responsible for the synthesis of the free fatty acids in the cutin biosynthetic pathway (Figure 4B). In addition to the mystery surrounding the order of the events in cutin biosynthesis, the mechanism for cutin polymerization outside of the cell wall remains uncharacterized. 
A)

Fatty acyl-CoAs

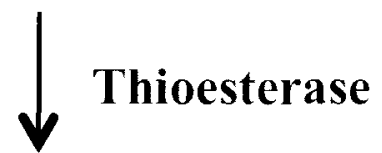

Fatty Acids

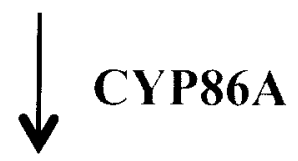

Hydroxylated FA

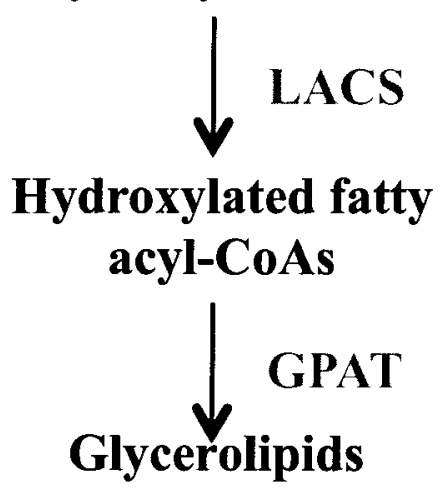

B)

Fatty acyl-CoAs

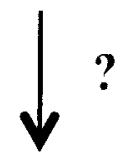

Fatty Acids

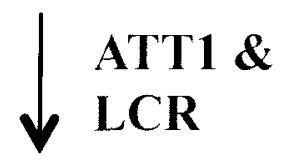

Hydroxylated FA

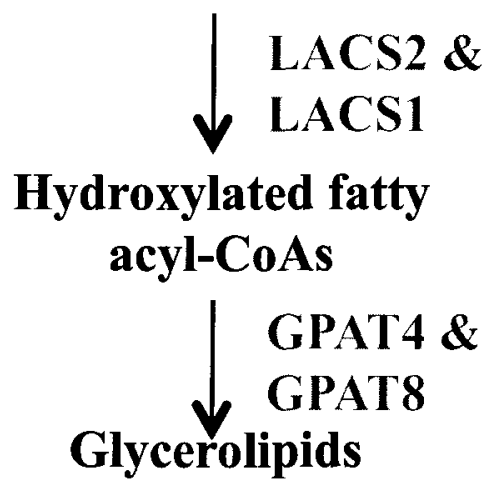

Figure 4: A model for cutin biosynthesis.

The understanding of the cutin biosynthetic pathway is in its infancy in comparison with the study of cuticular wax biosynthesis. (A) Some of the activities that occur in cutin synthesis are characterized, such as hydroxylation of fatty acids by cytochrome P450s and the transfer of these hydroxylated fatty acyl-CoAs to glycerol (Pollard et al., 2008). (B) Gene products from Arabidopsis that carry out some of these functions have recently been identified (Appendix 3). ATT1, ABERRANT INDUCTION OF TYPE THREE GENES; LCR, LACERATA; LACS, LONG-CHAIN ACYL-COA SYNTHASE; GPAT, GLYCEROL-3-PHOSPHATE ACYLTRANSFERASE. 
1.2.2 Cuticular wax synthesis

Cuticle biosynthesis occurs almost exclusively within the ER. Some of the $\mathrm{C} 16 / \mathrm{C} 18$ fatty acyl-CoAs in the ER are modified to form cutin monomers as previously described, while others are elongated to lengths between 20 and 32 carbons to generate the compounds in the cuticular wax. Elongation of fatty acyl-CoAs is carried out by the fatty acid elongase (FAE) complex at the ER membrane by a mechanism similar to that found during fatty acid synthesis (von Wettstein-Knowles, 1982; Post - Beittenmiller, 1996). The FAE complex catalyzes the elongation of the imported fatty acyl-CoAs through the addition of $\mathrm{C} 2$ units from malonyl-CoA instead of from malonyl-ACP, as is found in fatty acid synthesis (Fehling and Mukherjee, 1991). The elongation of the longchain fatty acyl-CoAs is controlled by the $\beta$-ketoacyl-CoA synthase, the first enzyme in the FAE complex. To date, four $\beta$-ketoacyl-CoA synthases have been reported to be associated with cuticle biosynthesis, each with its own specific substrate specificity (Miller and Kunst, 1997) (Appendix 3).

Following synthesis, very-long-chain fatty acyl-CoAs can be directed toward one of two major pathways: (1) the primary alcohol-forming pathway, or (2) the alkaneforming pathway (Figure 5) (Samuels et al., 2008). Very-long-chain fatty acyl-CoAs in the alcohol-forming pathway are reduced to primary fatty alcohols by CER4 and a subset of these primary alcohols are further esterified to form wax esters by WSD1 (Rowland et al., 2006; Li et al., 2008). In the alkane-forming pathway it is predicted that very-longchain fatty acyl-CoAs are reduced to form aldehydes, and subsequently decarbonylated to form alkanes (Cheesborough and Kolattukudy, 1984). CER1, CER3, and CER22 have been implicated in the biosynthesis of aldehydes and alkanes, but none of these predicted 
enzymes have been characterized sufficiently to elucidate the mechanism surrounding this step of the pathway (Rashotte et al., 2004; Rowland et al., 2007; Samuels et al., 2008). Within this pathway, the odd chain alkanes serve as substrates for MAH1. MAH1 is a mid-chain hydroxylase responsible for not only the synthesis of secondary alcohols, but also the synthesis of ketones (Greer et al., 2007). A portion of very-longchain fatty acyl-CoAs enter neither of these two pathways and are cleaved to form free fatty acids, which make-up approximately $3.2 \%$ of the total cuticular wax (Figure 5) (Jenks et al., 1995; Pollard et al., 2008). While no enzyme responsible for the synthesis of very-long-chain free fatty acids has been identified, the recently characterized CER8/LACS1 carries out the reverse reaction (Lü et al., 2009). CER8/LACS1 activates very-long-chain fatty acids with CoA to form very-long-chain fatty acyl-CoAs and is highly active for C30 acyl chains. These very-long-chain fatty acyl-CoAs can then be either further elongated or modified by one of the two primary wax biosynthetic pathways into other cuticular wax compounds (Lü et al., 2009; Weng et al., 2010). 


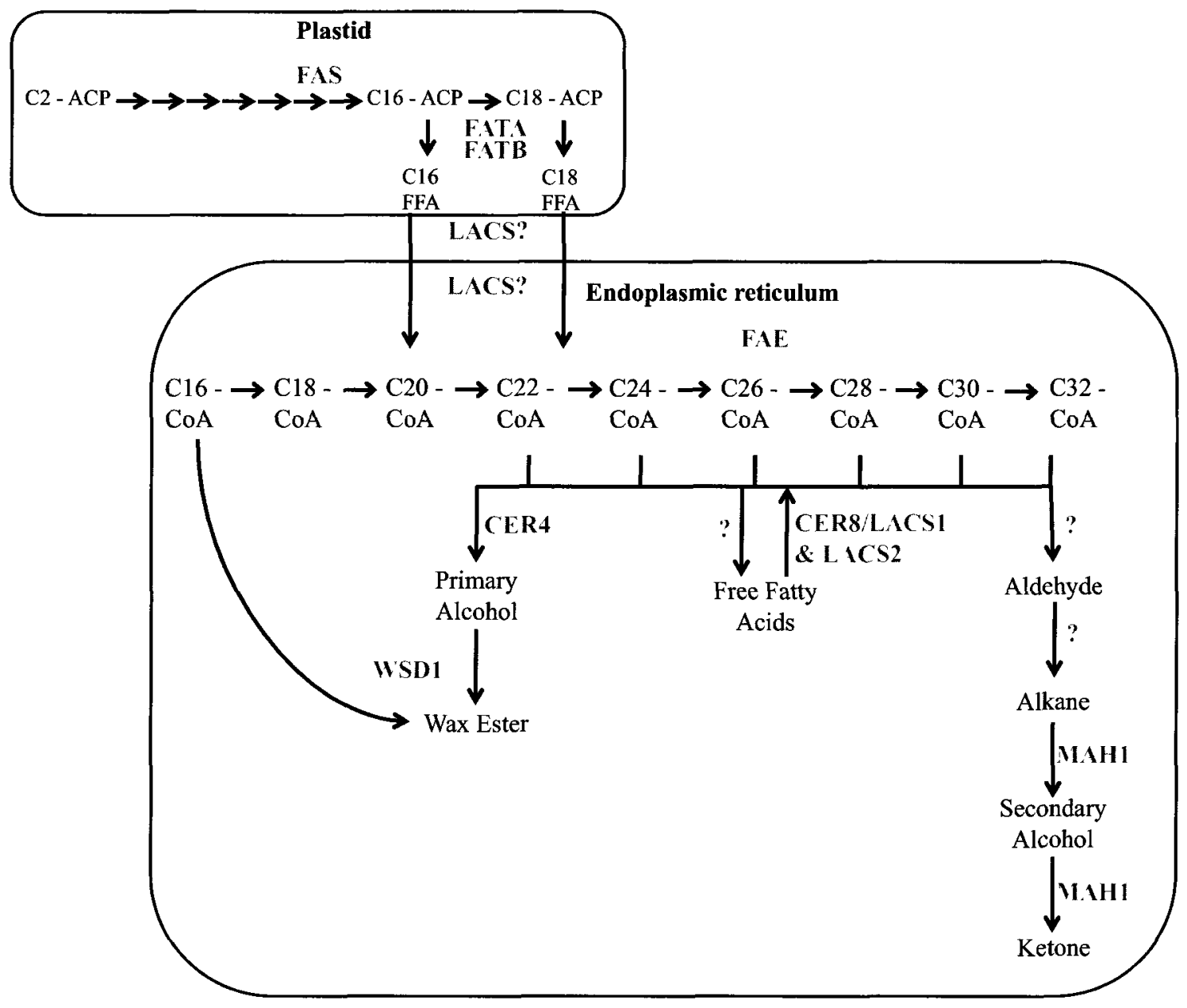

Figure 5: A simplified pathway for wax biosynthesis in Arabidopsis.

Enzymes functioning in several parts of the pathway have been well characterized such as CER4 (Rowland et al., 2006), WSD1 (Li et al., 2008), and MAH1 (Greer et al., 2007) (Appendix 3). In spite of this recent progress, our understanding of some aspects of the wax biosynthetic pathway remain limited. FAS, fatty acid synthase; FAT, fatty acylACP thioesterase; FFA, free fatty acid; LACS, long-chain acyl-CoA synthetase; FAE, fatty acid elongase; CER, ECERIFERUM; WSD, wax synthase/diacylglycerol acyltransferase; MAH, mid-chain alkane hydroxylase. 
1.3 Roles for thioesterases in lipid biosynthesis.

In spite of recent progress in the identification of cuticle biosynthetic genes (Appendix 3), several aspects have yet to be characterized such as alkane synthesis, aldehyde synthesis, and the production of free fatty acids. The cuticle contains verylong-chain free fatty acids and therefore fatty acyl-CoAs in the ER must be converted to free fatty acids (Figure 5). Cutin synthesis may also require the conversion of fatty acylCoAs to free fatty acids in order for hydroxylation to occur (Figure 4). For free fatty acids to be released from the fatty acyl-CoA substrates, the thioester bond between the CoA and the fatty acyl group must be hydrolyzed. This activity is typically carried out by a thioesterase (Figure 6). Two known plant acyl-ACP thioesterases, FATA and FATB, terminate fatty acid synthesis in the plastid (Salas and Ohlrogge, 2002). The FATB enzyme appears to be responsible for approximately $50 \%$ of cuticular wax precursors, while thioesterases contributing to the other $50 \%$ of precursors have not been identified (Bonaventure et al., 2003). The action of these additional thioesterases may be especially important in cells where lipid synthesis is high, such as in epidermal cells secreting large amounts of cuticular lipids. There are several potential roles for thioesterases in lipid biosynthesis including terminating fatty acid synthesis in the plastid, the formation of free fatty acids for the cuticular wax, the biosynthesis of cutin, and possibly as an indirect mechanism to regulate gene expression (also see Discussion). It is also possible that thioesterases are functioning in the biosynthesis of compounds that have yet to be identified within the cuticle. Only thioesterases functioning in fatty acid synthesis have thus far been identified. 


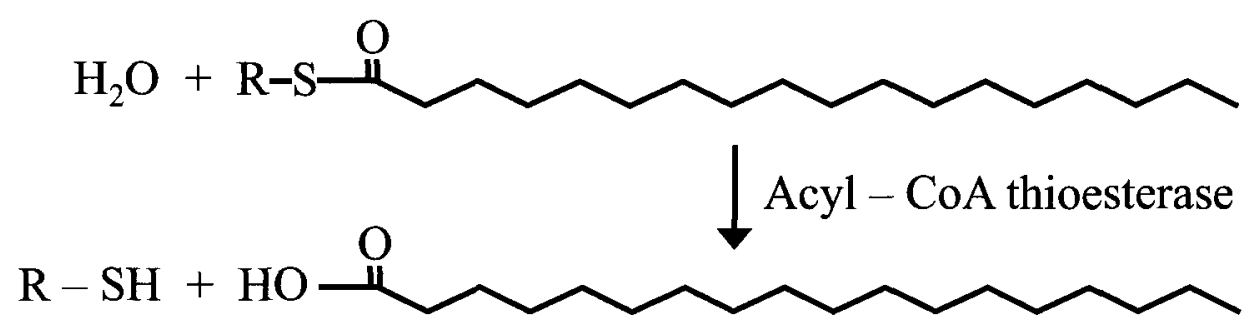

Figure 6: The fatty acyl thioesterase reaction.

Thioesterases cleave the thioester bond through the hydrolysis of a water molecule. $R$ can represent a $\mathrm{CoA}$ or $\mathrm{ACP}$ moiety. It can also represent a protein in the case of degrading acylated proteins. 
1.4 Families of thioesterases

Thioesterases are involved in a wide range of physiological roles such as lipid metabolism, aromatic compound degradation, acylated protein degradation, and signal transduction (Hunt and Alexson, 2002; Salas and Ohlrogge, 2002; Zhuang et al., 2003; Lu et al., 1996; Schujman et al., 2006; Hertz et al., 1998). There are two major superfamilies of thioesterases characterized to date, the $\alpha / \beta$ hydrolase and the Hotdog fold superfamilies. A recently developed database of enzymes that modify thioester bonds, ThYme, identifies 23 families of thioesterases based on the primary amino acid sequence (Cantu et al., 2010a). Of these 23 families, 8 belong to the $\alpha / \beta$ hydrolase superfamily and 12 belong to the Hotdog fold superfamily; accounting for approximately $87 \%$ of known thioesterases. While members of both superfamilies are capable of hydrolyzing the thioester bond, the three-dimensional structures of the two superfamilies are different (Cantu et al., 2010b).

\section{$1.4 .1 \alpha / \beta$ hydrolase superfamily}

The $\alpha / \beta$ hydrolase superfamily was initially identified by Ollis et al. (1992), who recognized that several unrelated proteins possess the same tertiary structure and conserved catalytic residues. Many of the members of this superfamily do not share primary sequence similarity, but the tertiary structure and arrangement of the catalytic site is remarkably similar (Ollis et al., 1992, Nardini and Dijkstra, 1999). Members of the $\alpha / \beta$ hydrolase superfamily carry out a variety of hydrolysis activities such as esterase and peroxidase reactions (Nardini and Dijkstra, 1999). The substrates of these enzymes are equally variable. Members of this superfamily can hydrolyze the thioester bond 
between long chain fatty acids and CoA in humans, while others function in the degradation of aromatic secondary metabolites, such as nicotine (Jones and Gould, 2000; Schleberger et al., 2007). The $\alpha / \beta$ hydrolase fold consists of a series of 8 parallel $\beta$ sheets surrounded by $\alpha$-helices, and typically exists as a monomer or dimer (Figure 7A). Between the various $\alpha / \beta$ hydrolase members, the number and position of the $\alpha$-helices can vary provided that the catalytic triad is unaltered and the parallel $\beta$-sheet conformation is conserved (Cantu et al, 2010b; Nardini and Dijkstra, 1999). This catalytic trio consists of a nucleophile, an acidic residue, and a histidine, all found on the loops between $\beta$-sheets (Figure 7B) (Ollis et al., 1992) (Nardini and Dijkstra, 1999). The majority of $\alpha / \beta$ hydrolase fold thioesterases characterized to date hydrolyze the thioester bond between an acyl chain and a protein, such as a fatty acid and an acyl carrier protein, or in the degradation of acylated proteins. Only 1 of the $8 \alpha / \beta$ hydrolase fold subfamilies that have thioesterase activity use CoA activated acyl chains as substrates (Cantu et al., 2010b). While this superfamily accounts for a significant portion of known thioesterases, acyl-CoA thioesterases appear to only account for a small portion of the $\alpha / \beta$ hydrolase fold superfamily. 
Figure 7: The $\alpha / \beta$ hydrolase fold is one of two major superfamilies with thioesterase activity.

This superfamily is characterized by series of 8 parallel $\beta$-sheets surrounded by loops and $\alpha$-helices (A). All types of hydrolases belonging to this family contain a conserved catalytic triad typically found on the loops between $\beta$-sheets consisting of a nucleophile (N), an acidic residue (A), followed by a histidine $(\mathrm{H})(\mathrm{A})$. Between individual proteins, the number and position of the $\alpha$-helices may vary provided the parallel series of sheets and catalytic residues are conserved (B). Arrows represent $\beta$-sheets and rectangles represent $\alpha$-helices in this model. Domains labeled in black illustrate some of the variations that have been identified from the typical $\alpha / \beta$ hydrolase model. Adapted from Nardini and Dijkstra (1999). 

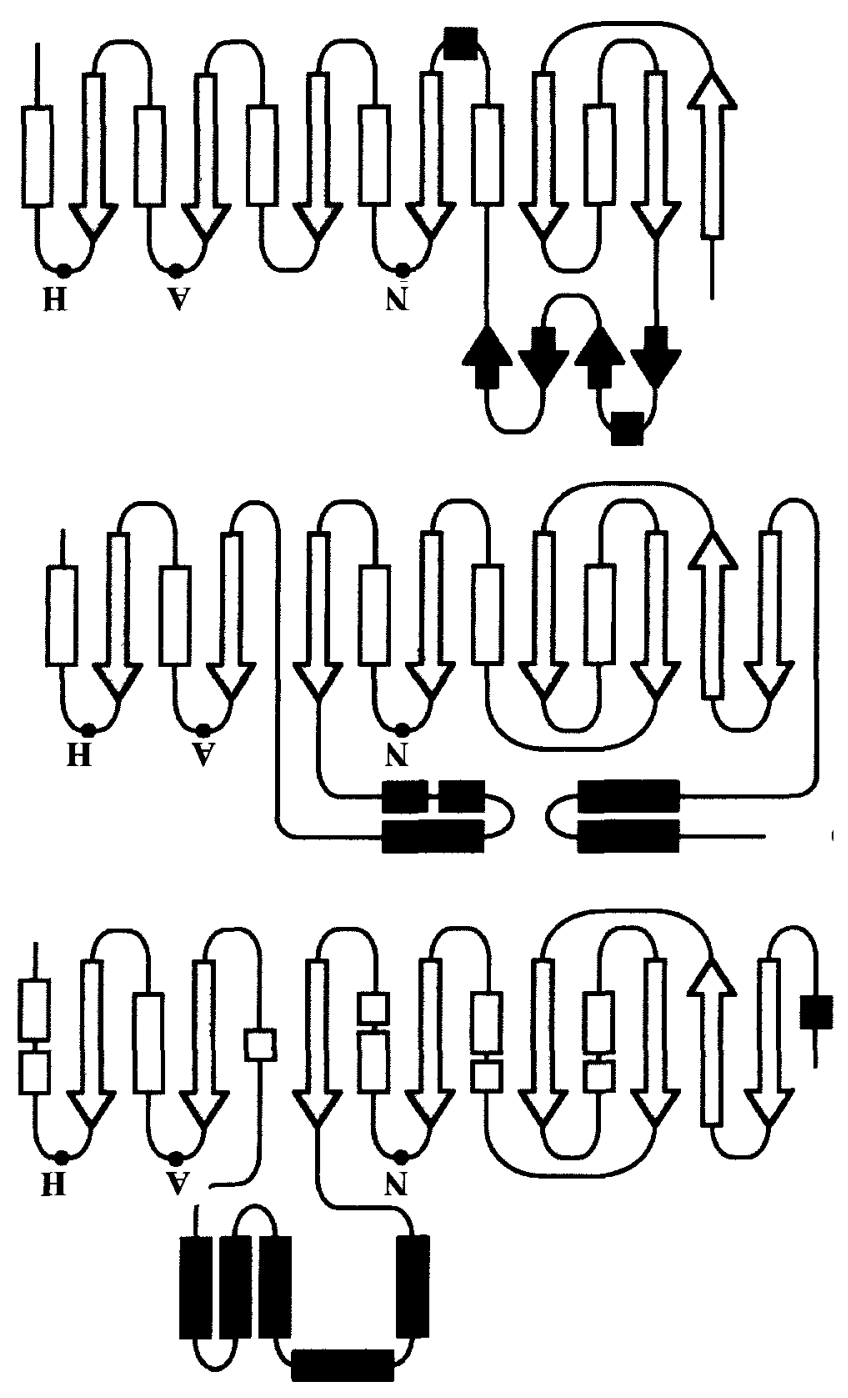

(g

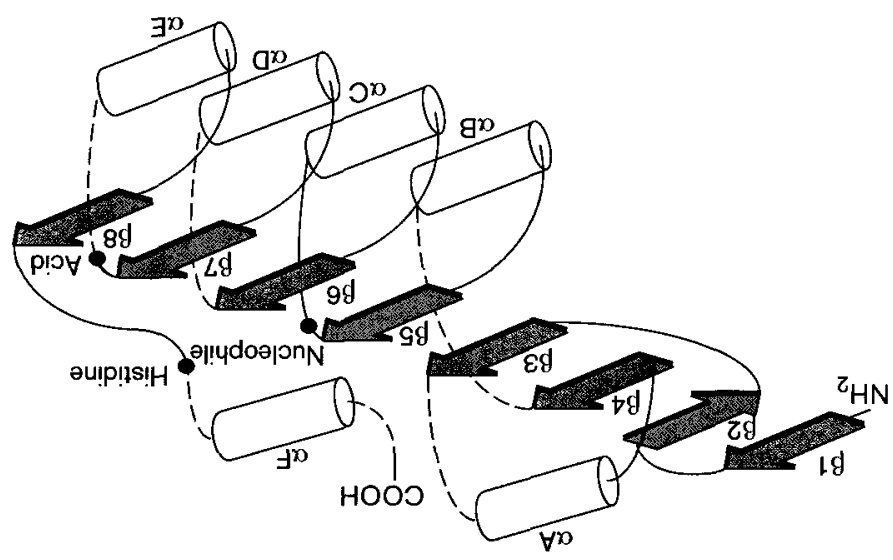


1.4.2 Hotdog fold superfamily

The Hotdog fold superfamily accounts for approximately half of known thioesterases. Unlike the $\alpha / \beta$ hydrolase fold, $83 \%$ of Hotdog fold thioesterases hydrolyze CoA thioester bonds (Cantu et al., 2010b). The Hotdog fold is characterized by a single $\alpha$-helix surrounded by a series of approximately $5 \beta$-sheets (Dillon and Bateman, 2004) (Figure 8A). In contrast to the multitude of activities observed in the $\alpha / \beta$ hydrolase fold superfamily, thus far all known Hotdog fold superfamily members catalyze only either thioesterase or dehydration reactions. The Hotdog fold was first described in the structure of the $E$. coli dehydratase FABA and the first Hotdog fold member identified with thioesterase activity was the 4-hydroxybenzoyl-CoA thioesterase 4HBT from Pseudomonas sp. Strain CBS-3 (Leesong et al., 1996; Benning et al., 1998). While the definition of an $\alpha / \beta$ hydrolase fold was dependent both on the location of specific catalytic residues and the tertiary structure, the Hotdog fold is defined only by tertiary structure. Each Hotdog fold subfamily possesses its own catalytic residues depending on the quaternary structure and substrate specificity of that specific subfamily (Pidugu et al., 2009). However, the catalytic residues of many of the Hotdog fold thioesterases involve an acidic residue (Pidugu et al. 2009; Zhuang et al., 2008; Kotaka et al., 2009). Hotdog fold proteins always involve the association of more than one Hotdog domain. This association can be through the interaction of two or more Hotdog domains within a single amino acid sequence or by the interaction of 2 monomeric subunits (Pidugu et al., 2009). Dimers typically form between two Hotdog domains in an anti-parallel manner such that one continuous $\beta$ - sheet is formed, while the $\alpha$ - helices run opposite of one another (Figure 8B) (Pidugu et al., 2009). If these dimers associate as tetramers, they do so in 
either a back-to-back orientation or through the central helix interaction (Figure $8 \mathrm{C}$ and D). Hexamers can form, but the associations between the subunits are more complex (Pidugu et al., 2009). The association of multiple Hotdog domains is important for the correct formation of the active site and binding pocket, and this is what provides the Hotdog fold superfamily the flexibility to hydrolyze a wide variety of substrates. The varying quaternary associations and variation within the superfamily contribute to the differences between the 1,4-dihydroxy-2-naphthoate-CoA thioesterase in the vitamin K biosynthetic pathway and FATA/FATB, which terminate Arabidopsis fatty acid synthesis (Widhalm et al., 2009; Salas and Ohlrogge, 2002). In spite of the lack of sequence identity, Hotdog fold proteins have a conserved structure and can have either thioesterase or dehydratase activity, accommodating a wide variety of substrates. 


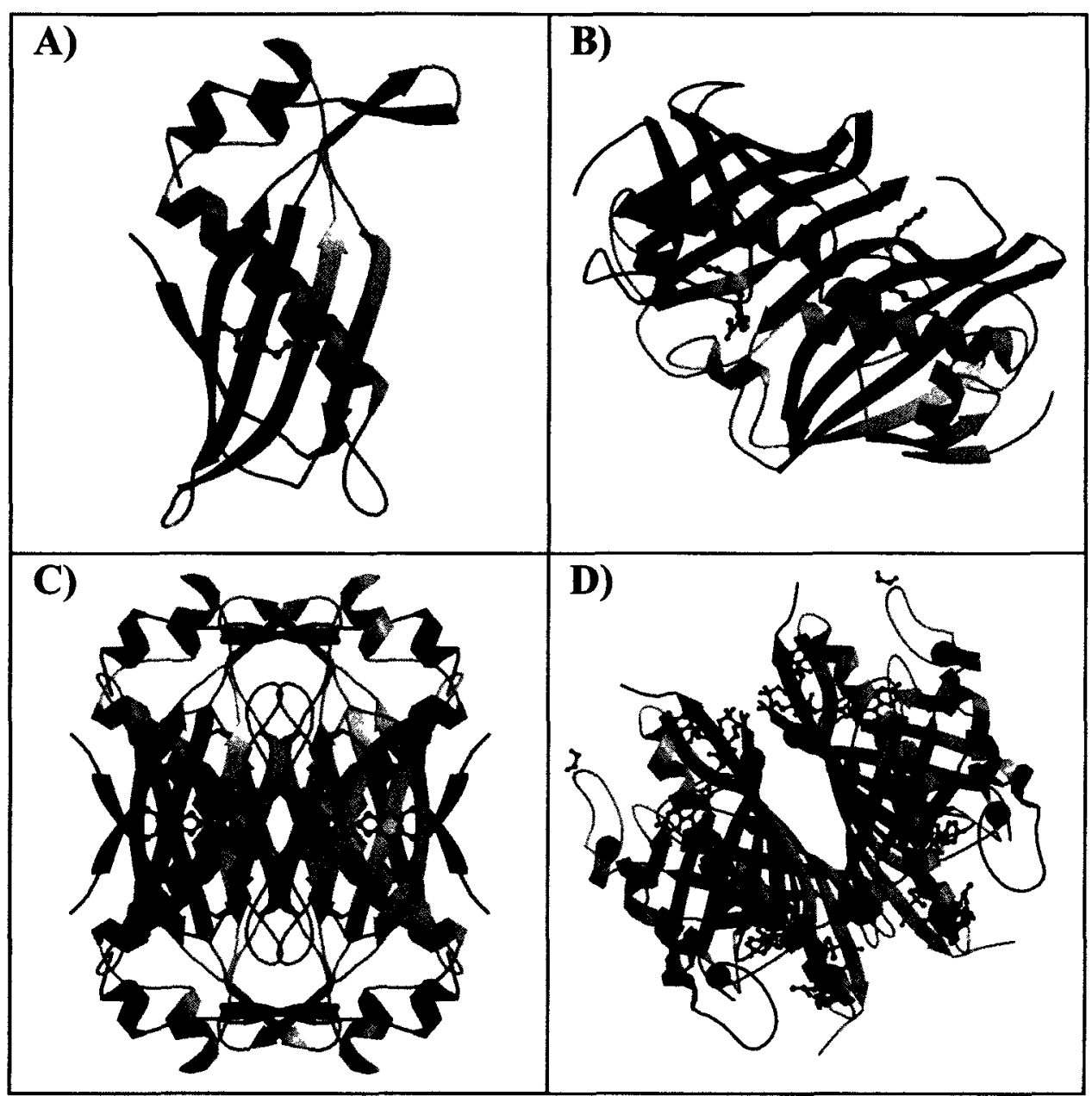

Figure 8: Conformations of the Hotdog fold superfamily.

The non-functional monomeric conformation of a Hotdog fold domain is an $\alpha$-helix surrounded by approximately $5 \beta$ - sheets (A) (Benning et al., 1998). The FABA E. coli dehydratase was the first identified Hotdog fold protein and assumes a dimeric conformation (B) (Leesong et al., 1996). Dimers commonly associate with one another to form tetramers through interactions between $\alpha$-helices, as is the case with the Pseudomonas sp. Strain CBS-3 4HBT (C) or in a back-to-back conformation found in the 4-hydroxybenzoyl-CoA thioesterase from Arthrobacter (D) (Benning et al., 1998;

Thoden et al, 2003). 
1.5 Thesis rationale

The purpose of this Masters thesis was to identify genes that encode thioesterases involved in cuticle biosynthesis in Arabidopsis thaliana. There are several potential roles for thioesterases in cuticle biosynthesis. Thioesterases could function in the release of very-long-chain fatty acids found in cuticular waxes. There is also a role for a thioesterase in one model of cutin monomer biosynthesis, in releasing long-chain free fatty acids for hydroxylation by cytochrome P450 enzymes. It is also possible that a novel thioesterase is working in parallel with FATB in terminating saturated fatty acid synthesis. There are also other putative roles for thioesterases in the regulation of lipid biosynthetic genes or in the production of lipid-associated secondary metabolites. I have identified a four member gene family that I have termed MODIFIERS OF EXTRACELLULAR LIPIDS (MEL) encoding thioesterases whose expression patterns are consistent with roles in extracellular lipid biosynthesis. I demonstrate that MEL1 has thioesterase activity in vitro toward palmitoyl-CoA. I also determine that in E. coli, MEL1, MEL2, and MEL3 are able to synthesize long-chain free fatty acids and mediumchain $\beta$-ketoacids. 


\section{Chapter 2: Materials and Methods}

2.1 Plant materials and growth conditions

All Arabidopsis thaliana plants were in the Columbia-0 background. Seeds were surface sterilized by incubation in $100 \%$ ethanol for 1 minute, followed by a 1 minute incubation in 50\% bleach (approximately $6 \%$ sodium hypochlorite) containing $0.5 \%$ SDS. Seeds were rinsed several times with sterile distilled water. Sterile seeds were sown on minimal media containing $5 \mathrm{mM} \mathrm{KNO}_{3}, 2.5 \mathrm{mM} \mathrm{KH}_{2} \mathrm{PO}_{4}, 2 \mathrm{mM} \mathrm{MgSO}_{4}, 2 \mathrm{mM}$ $\mathrm{Ca}\left(\mathrm{NO}_{3}\right)_{2}, 50 \mu \mathrm{M} \mathrm{Fe}(\mathrm{EDTA}), 70 \mu \mathrm{M} \mathrm{H}_{3} \mathrm{BO}_{3}, 14 \mu \mathrm{M} \mathrm{MnCl}_{2} \bullet 4 \mathrm{H}_{2} 0,0.5 \mu \mathrm{M} \mathrm{CuSO}_{4}, 1 \mu \mathrm{M}$ $\mathrm{ZnSO}_{4} \cdot 7 \mathrm{H}_{2} 0,0.2 \mu \mathrm{M} \mathrm{NaMoO}_{4} \bullet 2 \mathrm{H}_{2} 0,10 \mu \mathrm{M} \mathrm{NaCl}, 0.01 \mu \mathrm{M} \mathrm{CoCl}_{2} \bullet 6 \mathrm{H}_{2} 0$, and $0.7 \%$ agar (Somerville and Ogren, 1982). Seeds were then stratified in the dark for 3 to 5 days at $4^{\circ} \mathrm{C}$ and transferred to long-day conditions, 16 hours of light and 8 hours of dark, at $21^{\circ} \mathrm{C}$ (Conviron, Winnipeg MB) or to continuous-light conditions at room temperature. After approximately 14 days, seedlings were transferred to a soil-vermiculite mixture (ProMixMVP), fertilized with $1 \mathrm{~g} / \mathrm{L}$ 20-20-20 fertilizer (Plant-Prod, Brampton ON), and grown under long-day conditions at $22^{\circ} \mathrm{C}$ in a controlled growth chamber (Conviron or Percival chamber) or under continuous light in Nesbitt room 113A.

\subsection{Gene expression analysis by RT-PCR}

To determine in which tissues the $M E L$ genes were expressed, reverse transcription PCR (RT-PCR) was performed. Approximately $100 \mathrm{mg}$ each of flower, stem, and leaf was harvested from 4-week-old Arabidopsis and frozen in liquid nitrogen. Roots from 15-day-old seedlings was also harvested and frozen in liquid nitrogen. RNA was isolated from the tissues using TRIzol reagent (Invitrogen, Burlington $\mathrm{ON}$ ) as per 
the manufacturer's instructions. All solutions prepared in house were treated for 24 hours with diethylpyrocarbonate (DEPC) (Bioshop, Burlington ON) and autoclaved before use. First strand cDNA synthesis was carried out with $1 \mu \mathrm{g}$ of RNA and SuperScript III RT (Invitrogen) as per the manufacturer's specifications. A $0.4 \mu \mathrm{L}$ aliquot of cDNA from the various tissues was used as a template for PCR with pairs of gene specific primers: MEL1_RT_Fwd with, MEL1 RT_Rev, MEL2_RT_Fwd with MEL2_RT_Rev, and MEL3_RT_Fwd with MEL3_RT_Rev (Appendix 1). GLYCERALDEHYDE-3-PHOSPHATE DEHYDROGENASE (GAPC) levels were measured as a constitutive control using primers GAPC_Fwd and GAPC_Rev (Appendix 1). The PCR products were separated on a $1 \%$ agarose gel containing ethidium bromide and visualized using an AlphaImager 2200 (Alpha Innotech, Santa Clara CA).

\subsection{Cuticular wax analysis of Arabidopsis stems}

The cuticular wax composition of stem tissues was analyzed by gas chromatography and using a flame ionization detector (GC-FID). Approximately $8 \mathrm{~cm}$ of stem from 6-week-old plants grown under long day conditions was harvested just above the first internode. Samples were immersed in spectro grade chloroform (Caledon, Georgetown ON) containing $1 \mu \mathrm{g}$ of methyl heptadecanoate (Sigma-Aldrich) for 30 seconds. The chloroform was evaporated to dryness at $37^{\circ} \mathrm{C}$ under a gentle stream of nitrogen. The samples were dissolved in $\mathrm{N}, \mathrm{O}_{-}$ bis(trimethylsilyl)trifluoroacetamide:trimethylchlorosilane (BSTFA+TMCS; 99:1) (Sigma Aldrich), and derivatized at $80^{\circ} \mathrm{C}$ for 90 minutes. A $1 \mu \mathrm{L}$ aliquot was then analyzed by gas chromatography on a Varian 450-GC equipped with a HP-1 capillary 
column (15m x 250 $\mu \mathrm{m}$ i.d., 0.25 um film thickness, Varian, Santa Clara, CA) using helium as the carrier gas. The injector was held at $250^{\circ} \mathrm{C}$ and the oven held at a temperature of $150^{\circ} \mathrm{C}$ for 1 minute. The oven temperature was then raised at a rate of $4^{\circ} \mathrm{C}$ per minute to a final temperature of $300^{\circ} \mathrm{C}$. Finally, the oven was held at $300^{\circ} \mathrm{C}$ for 15 minutes.

\subsection{Cutin analysis of Arabidopsis thaliana}

About three mature rosette leaves and a comparable amount of stem tissue were harvested into chloroform rinsed glass vials for delipidation. The vials were filled with hot isopropanol and incubated at $80^{\circ} \mathrm{C}$ for 10 minutes. Once cooled, the isopropanol was replaced with a 2:1 (v/v) solution of chloroform:methanol for 24 hours. The samples were then incubated in 1:1 (v/v) chloroform:methanol, $1: 2(\mathrm{v} / \mathrm{v})$ chloroform:methanol, and $100 \%$ methanol for 24 hours each with changes twice a day. Samples were dried in the fumehood for 2 days and in a dessicator for a further 2 days (Domergue et al., 2010).

The dilipidated samples were sent to our collaborator, Dr. Frédéric Domergue, and the following procedure carried out in his laboratory. The dried tissues were depolymerized by incubation in $1 \mathrm{M}$ sulfuric acid in methanol and $2 \%(\mathrm{v} / \mathrm{v})$ dimethoxypropane for 3 hours at $85^{\circ} \mathrm{C}$. The samples were spiked with $10 \mu \mathrm{g}$ heptadecanoic acid, $10 \mu \mathrm{g} \omega$ - pentalactone, and $10 \mu \mathrm{g}$ pentadecanol as internal standards. Once cooled, $3 \mathrm{~mL}$ of $2.5 \% \mathrm{NaCl}$ was added to each sample and the lipids extracted with hexane. The hexane fraction was washed with $3 \mathrm{~mL}$ of saline solution $(200 \mathrm{mM} \mathrm{NaCl}$ and $200 \mathrm{mM}$ Tris, $\mathrm{pH} 8$ ) and then transferred to a fresh vial and dried under nitrogen. Samples were derivatized in $150 \mu \mathrm{L}$ of BSTFA+TMCS at $110^{\circ} \mathrm{C}$ for 30 minutes. The 
BSTFA+TMCS was evaporated under nitrogen and then the sample was dissolved in hexane for gas chromatography-mass spectrophotometry (GC-MS). GC-MS was carried out using an Agilent 6850 gas chromatograph with a HP-5MS column (30m X $0.25 \mathrm{~mm}$ $\mathrm{X} 0.25 \mu \mathrm{m})$ and an Agilent 5975 mass spectrometric detector $(70 \mathrm{eV}$, mass-to-charge ratio 50-750). The oven temperature was held at $50^{\circ} \mathrm{C}$ for 1 minute, increased at $25^{\circ} \mathrm{C}$ per minute to $150^{\circ} \mathrm{C}$, held at $150^{\circ} \mathrm{C}$ for 1 minute, increased by $10^{\circ} \mathrm{C}$ per minute to $320^{\circ} \mathrm{C}$, and held at $320^{\circ} \mathrm{C}$ for 8 minutes (method from Domergue et al. 2010).

\subsection{Ectopic expression of MEL1 in Arabidopsis.}

To ectopically express $M E L 1$, the cDNA sequence was cloned into a vector downstream of the cauliflower mosaic virus $35 \mathrm{~S}$ promoter. The MEL1 cDNA sequence was amplified from the clone U14921 (ABRC, Columbus OH) with primers MEL1_OE_XbaI_Fwd and MEL1_OE_SacI_Rev (Appendix 1) and iProof High-Fidelity DNA polymerase (Bio-Rad, Mississauga ON). The PCR product was digested with the restriction enzymes $X b a I$ and $S a c I$ (Invitrogen) and ligated with T4 DNA ligase (Invitrogen) into the vector pBAR containing the $35 \mathrm{~S}$ promoter to generate pBAR::35SMEL1 (Appendix 2). The product was also inserted between the $X b a \mathrm{I}$ and $S a c I$ sites in the $\mathrm{pVKH} 18$ vector containing a $35 \mathrm{~S}$ promoter with six enhancer sequences (Batoko et al., 2000). The pVKH18::35S-GFPN vector was digested with $X b a \mathrm{I}$ and SacI, excising the green fluorescent protein (GFP) coding sequence. The digested $\mathrm{pVKH} 18:: 35 \mathrm{~S}$ vector fragment was gel purified by the following phenol extraction procedure. Following electrophoresis, a UV box was used to image the DNA fragments and the large fragment associated with the pVKH18::35S vector was excised with a razor. The gel fragment was 
crushed with a syringe into a microcentrifuge tube and TE saturated phenol (Bioshop) added in a $1 \mu 1 / \mathrm{mg}$ of gel ratio. The mixture was vortexed for 1 minute before being frozen in liquid nitrogen. The aqueous layer was separated by centrifugation at 13,000 rpm for 15 minutes and the top layer transferred to a fresh microcentrifuge tube. Further purification of the DNA occurred by the addition of an equal volume of $25: 24: 1(\mathrm{v} / \mathrm{v})$ phenol:chloroform:isoamyl alcohol (Bioshop). After 1 minute of mixing, the sample was centrifuged at 13,000 rpm for 5 minutes. The aqueous layer was transferred to a fresh vial and the DNA ethanol precipitated with $3 \mathrm{M}$ sodium acetate. The $M E L 1$ coding sequence was ligated into $\mathrm{pVKH} 18:: 35 \mathrm{~S}$ between the corresponding restriction sites to generate pVKH18::35S-MEL1 (Appendix 2).

The vectors were transformed into chemically competent $E$. coli $\mathrm{DH} 5 \alpha$ cells. Five $\mu \mathrm{L}$ of each ligation was incubated with $50 \mu \mathrm{L}$ of thawed cells on ice for 30 minutes and heat shocked for 45 seconds at $45^{\circ} \mathrm{C}$. The cells were incubated at $37^{\circ} \mathrm{C}$ in $1 \mathrm{~mL}$ of LB media for 1 hour and plated on LB media with $1.5 \%$ agar containing $50 \mu \mathrm{g} / \mathrm{mL}$ kanamycin. E. coli colonies containing the plasmid with an insert were identified through colony PCR. Plasmids were then purified from $5 \mathrm{~mL}$ of overnight culture using a standard miniprep procedure, and the $M E L 1$ insert verified by DNA sequencing (Eurofin MWG Operon, Huntsville AL). Plasmids were transformed into GV3101 Agrobacterium tumefaciens cells by electroporation using a MicroPulser Electroporator (BioRad, Mississauga ON). The Agrobacterium was used to introduce the T-DNA regions of the binary plasmids into wild-type plants by the floral dip method (Zhang et al., 2006). Successful Arabidopsis transformants containing the T-DNA harbouring 35SMEL1 were selected on minimal media containing $30 \mu \mathrm{g} / \mathrm{mL}$ hygromycin (Bioshop). 
Arabidopsis containing the T-DNA harbouring 35S-MEL1 were selected with a 1:1000 dilution of Finale (Farnam Companies Inc, Phoenix AZ), which was sprayed on seedlings 4, 7, and 10 days after sowing. Positive transformants were transplanted to soil and after approximately 4 weeks of growth stem samples were harvested for cuticular wax as described above.

\subsection{Generation of artificial microRNA lines}

To decrease the transcript levels of $M E L 1$, artificial microRNAs (amiRNAs) were generated using the miR319a Arabidopsis microRNA backbone and overlapping PCR to selectively change nucleotide residues (Figure 9) (Ossowski et al., 2008). MEL1 specific amiRNA sequences were identified with WMD2-Web MicroRNA Designer to generate amiRNA lines A and B (Ossowski et al., 2005) (Ossowski et al., 2008). Based on these amiRNA sequences, the WMD2 tool provided the amiRNA specific primers MEL1_amiA_I, MEL1_ami-A_II, MEL1_ami-A_III, and MEL1_ami-A_IV in the case of MEL1_amiRNA-A, and MEL_ami-B_I, MEL_ami-B_II, MEL_ami-B_III, and MEL_ami-B_IV in the case of MEL1_amiRNA-B (Appendix 1). In addition to these amiRNA specific primers, the general primers pRS300_A and pRS300_B (Appendix 1) were used to complete the overlap PCR reactions outlined in Table 1 (Figure 9) with iProof High Fidelity Polymerase to generate MEL1_amiRNA-A and MEL1_amiRNA-B.

Each of the first 3 reactions was gel-purified from a 1\% agarose gel using the phenol extraction procedure described above, and these were used as a template for reaction 4 . The reaction 4 amplified product was digested with the restriction enzymes EcoRI and BamHI (Invitrogen) and ligated between the corresponding sites in the 
pBluescript vector to generate pBluescript-MEL1_amiRNA-A and pBluescript-

MEL1_amiRNA-B. The ligation mixtures were transformed into chemically competent

DH5 $a$ and clones containing inserts were identified. The inserts were sequenced to make sure there were no unintended mutations. Adapter primers, Ami_adapterA_XbaI and Ami_adapterB_Sacl (Appendix 1), were used to amplify by PCR the amiRNAs from pBluescript appending $X b a \mathrm{I}$ to the 5' end and SacI to the 3' end of the amiRNAs with iProof High Fidelity polymerase. The products were then cloned into the corresponding sites of pBAR::35S and pVKH18::35S to create the constructs pBAR::35SMEL1_amiRNA-A, pVKH18::35S-MEL1_amiRNA-A, pBAR::35S-MEL1_amiRNA-B, and pVKH18::35S-MEL1_amiRNA-B (Appendix 2). Following verification of the DNA sequence, the vectors were transformed into GV3101 Agrobacterium tumefaciens cells by electroporation and wild-type Arabidopsis were transformed by the floral dip method (Zhang et al., 2006). Selection and analysis of transformants was carried out as previously described for the MEL1 over-expression plants. 
Table 1: PCR strategy to synthesize amiRNA sequences specific for $M E L 1$

Summary of the PCR strategy showing the primers and template used for each of the amplifications to form the full-length amiRNA sequences (Adapted from Ossowski et al., personal communication, 2005). The $\mathrm{X}$ is used in place of $\mathrm{A}$ or $\mathrm{B}$ in the primer names as this strategy applies to the synthesis of both MEL1_amiRNA-A and MEL1_amiRNA-B.

\begin{tabular}{|l|l|l|}
\hline \multicolumn{1}{|c|}{ Reaction } & \multicolumn{1}{|c|}{ Primers } & \multicolumn{1}{c|}{ Template } \\
\hline Reaction 1 & $\begin{array}{l}\text { pRS300_A } \\
\text { MEL1_ami-X_IV }\end{array}$ & pRS300 \\
\hline Reaction 2 & $\begin{array}{l}\text { MEL1_ami-X_III } \\
\text { MEL1_ami-X_II }\end{array}$ & pRS300 \\
\hline Reaction 3 & $\begin{array}{l}\text { MEL1_ami-X_I } \\
\text { pRS300_B }\end{array}$ & pRS300 \\
\hline Reaction 4 & $\begin{array}{l}\text { pRS300_A } \\
\text { pRS300_B }\end{array}$ & Reactions 1, 2, and 3 \\
\hline
\end{tabular}




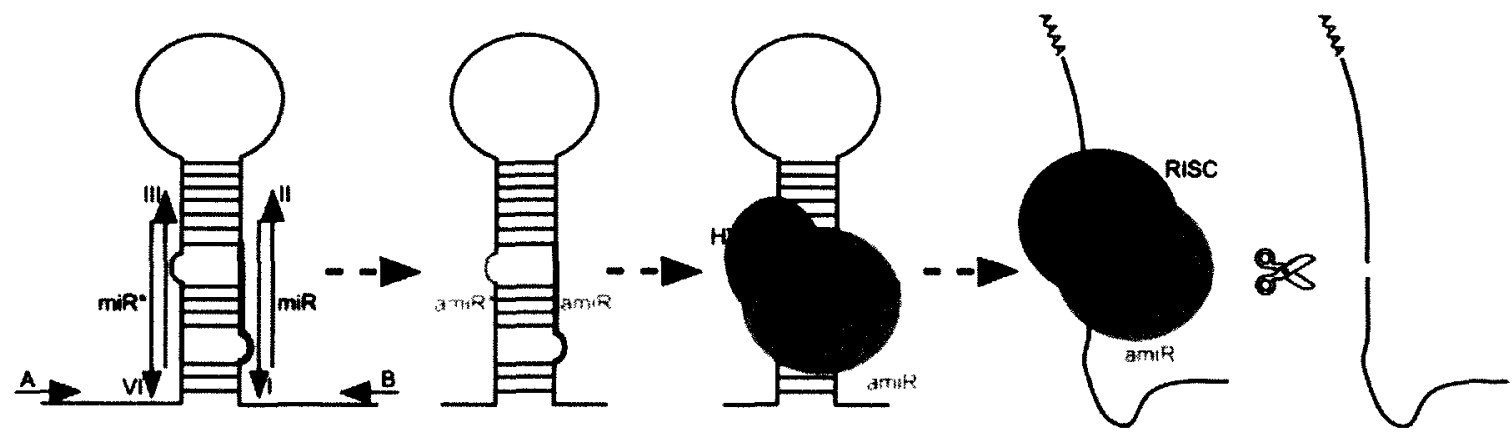

Figure 9: A model for artificial amiRNA synthesis and how amiRNAs silence genes of interest.

Following overlapping PCR, a hairpin RNA sequence is synthesized with specific mutations which may the artificial microRNA specific for the gene of interest. RNA processing machinery within the plant recognizes the double-stranded RNA within the hairpin structure and cleaves the RNA. The small fragments of RNA are then free to anneal to the targeted transcipt. This again induces RNA processing to cleave the double-stranded RNA including the mRNA template (Ossowski et al., 2005). 
2.7 In situ hybridization for the detection of $M E L 1$ transcript

An antisense probe specific for $M E L 1$ was amplified from the clone U14921 (ABRC) containing the cDNA sequence for $M E L 1$ using the primers

MEL1_Antisense_Fwd and MEL1_Antisense_Rev (Appendix 1) that appends the T7 polymerase binding site to the 5' end of the template strand. As a negative control, a sense probe was also amplified from the same clone using the primers MEL1_sense_Fwd and MEL1_sense_Rev (Appendix 1). Amplification was carried out with Taq polymerase and the resulting PCR product purified using the Wizard SV Gel and PCR Clean up kit (Promega, Madison WI). Transcription of digoxigenin labeled RNA probes was carried out with DIG RNA Labeling Kit (SP6/T7) (Roche, Mannheim Germany) using the sense and antisense templates. The probe was then ethanol precipitated with $4 \mathrm{M} \mathrm{LiCl}$ and resuspended in $100 \mu \mathrm{L}$ of DEPC treated water. The full-length RNA probe was subject to carbonate-mediated hydrolysis by incubating the probe with $60 \mathrm{mM}$ $\mathrm{NaHCO}_{3}$ and $40 \mathrm{mM} \mathrm{Na}_{2} \mathrm{CO}_{3}$ for 27 minutes at $60^{\circ} \mathrm{C}$, followed by ethanol precipitation with $3 \mathrm{M}$ sodium acetate. The probe was resuspended in $50 \%$ deionized formamide and quantified as outlined by the manufacturer's instructions in the DIG RNA Labeling Kit (SP6/T7) and the DIG Nucleic Acid Detection Kit (Roche). The probe was quantified by spotting the probe onto a nylon membrane and then imaged using an Alphalmager 2200 (Alpha Innotech).

Stem tissue for in situ hybridization was harvested from 6-week-old wild-type plants and fixed in formol-acetic-alcohol (50\% ethanol, $5 \%$ acetic acid, $3.7 \%$ paraformaldehyde) (Canemco, Lakefield QC), in a glass scintillation vial (Huijzer et al., 1992). The tissues immersed in fixative were repeatedly placed in a vacuum at 15 
minutes intervals until all tissue had sunk to the bottom of the vial, after which the fixation was allowed to proceed for 2 hours at room temperature. The samples were washed with $50 \%$ ethanol and incubated in fresh $50 \%$ ethanol for 30 minutes. Samples were dehydrated through 30 -minute incubations in each of $50 \%, 60 \%, 70 \%, 85 \%$, and $95 \%$ ethanol, and then incubated in $95 \%$ ethanol with $0.1 \%$ eosin overnight. Samples were incubated in $100 \%$ ethanol for 2 hours with changes for fresh $100 \%$ ethanol after 60 and 90 minutes. The tissue was infiltrated with xylene (Fisher Scientific, Pittsburgh PA) through 30-minute incubations in increasing concentrations $(25 \%, 50 \%$, and $75 \%)$ of xylene in ethanol followed by a 1-hour incubation in $100 \%$ xylene with a change to fresh xylene after 30 minutes. Approximately 20 paraplast (Sigma-Aldrich) chips were added to the vial and the samples incubated overnight at room temperature. The vial was placed in a $42^{\circ} \mathrm{C}$ waterbath for 1 hour to melt the paraplast chips, and moved to the $60^{\circ} \mathrm{C}$ oven for 4 hours. The paraplast- xylene mix was changed for $100 \%$ melted paraplast chips. The paraplast was replaced with fresh melted chips twice daily for 3 days. Following 6 wax changes, the infiltrated tissues and wax was poured into a petri dish floating in a $60^{\circ} \mathrm{C}$ water bath. Samples were arranged with dissecting needles and the temperature lowered allowing the paraplast to solidify. The samples were cut from the paraplast block and mounted onto small wooden blocks and $8 \mu \mathrm{m}$ cross sections of stem were sectioned with a rotary microtome (ThermoFisher Scientific, Waltham MA) and placed on frosted slides (Superfrost Plus, Fisher Scientific). The sections were bonded to the slides by incubating the slides at $42^{\circ} \mathrm{C}$ overnight on a slide warmer.

Slide preparations were dewaxed by 2 incubations of 10 minutes in $100 \%$ xylene. Rehydration was performed by incubating the slides twice in $100 \%$ ethanol for 2 minutes, 
followed by incubations in $95 \%$ and $90 \%$ ethanol, and $80 \%, 60 \%$, and $30 \%$ ethanol containing $0.85 \% \mathrm{NaCl}$ for 1 minute per solution. Slides were incubated in $0.85 \% \mathrm{NaCl}$ and phosphate buffered saline (PBS) for 2 minutes. Slides were then subjected to proteinase $\mathrm{K}$ digestion by incubation in $1 \mu \mathrm{g} / \mathrm{mL}$ proteinase $\mathrm{K}$ (Sigma-Aldrich), $100 \mathrm{mM}$ TRIS ph 7.5, and $50 \mathrm{mM}$ EDTA for $30 \mathrm{~min}$ and incubated for 2 minutes each in $0.5 \mathrm{~g} / \mathrm{L}$ glycine in PBS and PBS. Slides were postfixed in 4\% paraformaldehyde in PBS for 2 minutes followed by a 2 min rinse in PBS. Samples were acetylated by incubating slides for 2 minutes in approximately $100 \mathrm{mM}$ triethanolamine and $30 \mathrm{mM}$ acetic anhydride, followed by 2- 5 minute washes in PBS and a 5 minute wash in $0.85 \% \mathrm{NaCl}$. Slides were dehydrated by dipping slides in each of $0.85 \% \mathrm{NaCl}, 30 \%$ ethanol, $60 \%$ ethanol, $80 \%$ ethanol, and $90 \%$ ethanol containing $0.85 \% \mathrm{NaCl}$, and $95 \%$ ethanol, and 2 rinses in $100 \%$ ethanol for 30 seconds each.

Hybridization with the probe was performed with $150 \mu \mathrm{L}$ of hybridization buffer (50\% formamide, 10\% dextran sulfate (Sigma-Aldrich), 1X Denhardt's (Sigma-Aldrich), $20 \mathrm{mg} / \mathrm{mL}$ tRNA(Roche), $10 \mathrm{mM}$ Tris $\mathrm{pH} 7.5,1 \mathrm{mM}$ EDTA, and $300 \mathrm{mM} \mathrm{NaCl}$ ) combined with 5, 10 and $20 \mathrm{ng}$ of antisense probe, $5 \mathrm{ng}$ of sense probe for a negative control, and $5 \mathrm{ng}$ of CER6 probe for a positive control (CER6 probe synthesized and provided by Mingli Xu, Ph.D candidate, Dr. Hepworth lab; described in Hooker et al., 2002). Probes were diluted in $50 \%$ formamide to a final volume of $50 \mu \mathrm{L}$, such that the final ratio of hybridization buffer to probe was 3:1 (Samach et al., 1997). The probe hybridization solution was heated to $80^{\circ} \mathrm{C}$ for 2 minutes and then placed on ice. All the sample slides were placed on the slide warmer at $52^{\circ} \mathrm{C}$, and $150 \mu \mathrm{L}$ of the hybridization buffer containing the probe was applied to each slide and a cover slip lowered to cover all 
samples without causing air bubbles. The slides were placed on risers in a box prewarmed to $55^{\circ} \mathrm{C}$ with Whatman paper soaked in $2 \mathrm{X} \mathrm{SSC}(300 \mathrm{mM} \mathrm{NaCl}$ and $30 \mathrm{mM}$ sodium citrate dihydrate) in $50 \%$ formamide. The box was sealed in a plastic bag with water soaked paper towels and left at $55^{\circ} \mathrm{C}$ overnight. To wash excess probe from the slides, samples were transferred to $2 \mathrm{x}$ SSC for 5 minutes at $55^{\circ} \mathrm{C}$ dipping slides up and down until cover slips were removed. Slides were incubated in $0.2 \mathrm{X} \mathrm{SSC}$ at $55^{\circ} \mathrm{C}$ for 2 hours with a change of solution every 30 minutes. They were then transferred to $0.2 \mathrm{X}$ $\mathrm{SSC}$ at $37^{\circ} \mathrm{C}$ for 5 minutes and finally $0.2 \mathrm{X} \mathrm{SSC}$ at room temperature for $5 \mathrm{~min}$. The slides were stored in a plastic container containing PBS over night at $4^{\circ} \mathrm{C}$. Each slide was incubated for 1 hour in $1 \mathrm{~mL}$ of $1 \%$ blocking solution (Roche) in maleic acid. Slides were washed with $2 \mathrm{~mL}$ of wash solution (1\% BSA, $0.3 \%$ Triton-X100, $100 \mathrm{mM}$ Tris $\mathrm{HCl} \mathrm{pH} \mathrm{7.5,} \mathrm{and} 150 \mathrm{mM} \mathrm{NaCl}$ ) per slide, for 1 hour. The anti-digoxigenin-AP, Fab fragments (Roche) were diluted 1:1250 in the wash solution and $250 \mu \mathrm{L}$ of the diluted antibody solution was added to each slide under a cover slip and incubated for 90 minutes at room temperature (Coen et al., 1990). The excess antibody was removed by rinsing the slides 3 times in wash solution for intervals of 20 minutes each. Slides were placed in $100 \mathrm{mM}$ TRIS-HCl (pH 9.5), $500 \mathrm{mM} \mathrm{NaCl}$, and $100 \mathrm{mM} \mathrm{MgCl}_{2}$ for 30 minutes changing solutions after 15 minutes. The colourimetric reaction was carried out by the addition of $250 \mu \mathrm{L}$ of $18 \% \mathrm{BCIP} / \mathrm{NBT}$ in $100 \mathrm{mM}$ TRIS- $\mathrm{HCl} \mathrm{pH} 9.5,500 \mathrm{mM} \mathrm{NaCl}$, and $100 \mathrm{mM} \mathrm{MgCl}_{2}$ to each slide. The reaction proceeded overnight in the dark and was stopped by placing the slides in $10 \mathrm{mM}$ TRIS- $\mathrm{HCl} \mathrm{pH} 8$ and $10 \mathrm{mM}$ EDTA, and imaged under 50\% glycerol using an Axio Imager M2 (Zeiss, Thornwood NY). All solutions 
used for in situ hybridization except for the ones containing TRIS were treated with DEPC for 24 hours and autoclaved before use.

2.8 Promoter-glucuronidase (GUS) fusions and the GUS histochemical assay

The expression patterns of $M E L 1, M E L 2, M E L 3$, and $M E L 4$ were examined using the $\beta$ - glucuronidase (GUS) reporter system. Approximately $1.8 \mathrm{~Kb}$ of sequence upstream of the start codon of each of each gene was amplified from the BAC clones containing genomic DNA. The BACs T9I1, containing MEL1 and MEL2, and T22E19 containing, MEL3 and MEL4, (ABRC), were used as templates with the following primers: MEL1_Prom_SalI_Fwd with MEL1_Prom_BamHI_Rev, MEL2_Prom_Sall_Fwd with MEL2_Prom_BamHI_Rev, MEL3_Prom_SalI_Fwd with MEL3_Prom_BamHI_Rev, and MEL4_Prom_SalI_Fwd with MEL4_Prom_BamHI_Rev (Appendix 1) using iProof High-Fidelity DNA polymerase. The PCR products were digested with the restriction enzyme SalI (Invitrogen) and BamHI and ligated between the corresponding sites in the $\mathrm{pBI} 101.1$ vector and in frame with the GUS coding region to generate pBI101-MEL1 promoter GUS, pBI101-MEL2 promoter GUS, pBI101-MEL3 promoter GUS, and pBI101-MEL4 promoter GUS. These vectors were transformed into DH5 $\alpha$ cells as previously described, and the amplified region sequenced to confirm that there were no PCR-induced errors. The plasmids were then transformed into GV3101 Agrobacterium cells by electroporation. Agrobacterium was then used to transform wildtype plants by the floral dip method (Zhang et al., 2006). I acknowledge Swara Narayanan for the construction of these plasmids. Successful transformants were identified by selection on minimal media containing $50 \mu \mathrm{g} / \mathrm{mL}$ kanamycin, as described 
previously. These were transplanted to soil and at about 4 weeks of age various aerial tissues were stained to identify lines with appropriate staining patterns. GUS staining of aerial tissues was carried out by immersing tissue in heptane for a few minutes and then rinsing with pre-chilled staining buffer, $50 \mathrm{mM} \mathrm{NaH}_{2} \mathrm{PO}_{4}, 100 \mathrm{mM} \mathrm{KFe}(\mathrm{CN})_{6}$, and $0.1 \%$ Triton X-100. Samples were incubated in staining solution, which is staining buffer plus $1 \mathrm{mM} \mathrm{X}$-Glucuronide (Gold Biotechnologies, St. Louis $\mathrm{MO}$ ), at $37^{\circ} \mathrm{C}$ for $2-18$ hours depending on the individual line and tissue type. The staining solution was then replaced with $70 \%$ ethanol and samples imaged with a Zeiss SteREO Discovery V20 (Zeiss). Seeds from transgenic lines showing consistent staining patterns were harvested and sown on selectable media. Following transplantation, samples from these plants were harvested at various ages and stained to further characterize the expression pattern of the reporter genes driven by the upstream regions of the $M E L$ genes. Positive transgenics were also transplanted to non-selectable media and grown vertically on plates for several days. Root tissue was stained by incubating samples GUS staining solution for approximately 2 hours at $37^{\circ} \mathrm{C}$ and stored in $70 \%$ ethanol.

Stained tissues were embedded in paraffin and cross-sectioned using a rotary microtome as described in section 2.7. The slides were prepared as described previously, except following rehydration samples were mounted under $50 \%$ glycerol. A cover slip was applied and samples imaged with an Axio Imager.M2. Slides were sealed with nail polish for long-term storage. I acknowledge Swara Narayanan, Carlos Canez, and Sollapura Vishwanath for analyzing and photographing the MEL promoter:GUS lines. 
2.9 Plasmid constructs for expression of MELs in E. coli

The cDNA sequences for $M E L 1, M E L 2$, and $M E L 3$ were amplified from clones U14921 (MEL1), U84163 (MEL2), and U14083 (MEL3) obtained from ABRC using iProof High-Fidelity DNA polymerase and primers: MEL1_BamHI_Fwd with MEL1_EcoRI_Rev, MEL2_BamHI_Fwd with MEL2_EcoR1_Rev, and MEL3_BamHI_Fwd with MEL3_EcoRI_Rev (Appendix 1). PCR products were digested with $B a m \mathrm{HI}$ and $E c o$ RI restriction enzymes and ligated between the corresponding sites of the pET28a vector (Novagen, Darmstadt Germany), which contains a region encoding an $\mathrm{N}$-terminal His $6 \mathrm{X}$ tag and a $\mathrm{T} 7$ epitope tag, to generate pET28a-MEL1, pET28a-MEL2, and pET28a-MEL3 (Appendix 2). I acknowledge Xiaoxue Wen and Dr. Owen Rowland for building the pET28a-MEL2 and pET28aMEL3 constructs. To generate a non-active version of MEL1, the codon for aspartic acid residue at position 64 was mutated to a codon encoding an alanine. For this, the cDNA sequence for MEL1 was amplified with mutagenic primers in 2 segments with iProof High-Fidelity DNA polymerase from the clone U14921 (ABRC). The first reaction contained primers MEL1_BamHI_Fwd and MEL1_D64A_Rev and the second reaction contained MEL1_D64A_Fwd and MEL1_EcoR1_Rev (Appendix 1). The resulting fragments were gel purified by the phenol extraction procedure, as described in section 2.5, and used as the template for overlapping PCR with the primers MEL1_BamHI_Fwd and MEL1_EcoRI_Rev (Heckman and Pease, 2007). The resulting product was then digested with $B a m \mathrm{HI}$ and $E c o \mathrm{RI}$ and ligated between the corresponding sites of pET28a to generate pET28a-MEL1(D64A) (Appendix 2). 
The FATB open reading frame lacking the region coding for the chloroplast transit sequence was amplified from flower cDNA using iProof High-Fidelity DNA polymerase with the primers FatB_Trunc_SacI_Fwd and FatB_HindIII_Rev (Appendix 1) (Mayer and Shanklin, 2007). This was then digested with SacI and HindIII restriction enzymes and ligated into the pET28a vector to generate pET28a-FATB (Appendix 2).

Plasmids containing MEL1, MEL1 (D64A), MEL2, MEL3, and FATB were transformed into E. coli BL21(DE3)pLysS cells (Promega, Madison WI) by electroporation. These constructs were also transformed into chemically competent K27(DE3), which was created by former Rowland Lab M.Sc .student Tao Song (Lü et al., 2009). The E. coli cell line K27 has a knock-out mutation of the acyl-CoA synthetase FadD gene. The K27(DE3) lysogen contains a chromosomally integrated T7 RNA Polymerase coding sequence under the control of the lacUV5 promoter, which allows for inducible expression of genes cloned downstream of the T7 promoter on the $\mathrm{pET}$ plasmid. As a negative control, an empty pET28a vector was transformed into each of these cell lines.

\subsection{Media analysis}

To analyze the media for the synthesis of novel lipid compounds, K27(DE3) cells with empty pET28a or pET28a vectors containing MEL1, MEL2, MEL3, MEL1(D64A), or $F A T B$ were inoculated into $5 \mathrm{~mL}$ of $\mathrm{LB}$ containing $50 \mu \mathrm{g} / \mathrm{mL}$ kanamycin. These cultures were grown at $37^{\circ} \mathrm{C}$ overnight. Larger $50 \mathrm{~mL}$ cultures of LB containing 50 $\mu \mathrm{g} / \mathrm{mL}$ kanamycin were inoculated with $1 \mathrm{~mL}$ of the overnight culture and grown at $30^{\circ} \mathrm{C}$ with shaking at $200 \mathrm{rpm}$ until the optical density $(\lambda=600 \mathrm{~nm})$ of the culture was between 
0.5 and 0.7 . The cells were induced with $1 \mathrm{mM}$ isopropyl $\beta$-D-1-thiogalactopyranoside (IPTG) (Bioshop) and grown at $18^{\circ} \mathrm{C}$ overnight. The culture was pelleted in a chloroform rinsed glass vial by centrifugation at $5000 \mathrm{rpm}$ for 15 minutes in an Eppendorf centrifuge 5804R with an A4-4-4 rotor (Eppendorf, Mississauga ON).

To analyze the LB media for the presence of free fatty acids, $0.5 \mathrm{~mL}$ of the media from the pelleted culture was transferred to a fresh glass vial. $1 \mathrm{~mL}$ of $1: 1(\mathrm{v} / \mathrm{v})$ chloroform:methanol and $22 \mu \mathrm{L}$ of glacial acetic acid was added to the media and the solution mixed by inversion. Samples were centrifuged at $5000 \mathrm{rpm}$ for 10 minutes and the lower chloroform phase transferred to a fresh GC vial. The chloroform was evaporated under nitrogen and $1 \mathrm{~mL}$ of $2 \%$ sulfuric acid in methanol was added and the sample incubated at $90^{\circ} \mathrm{C}$ for 60 minutes. Once cooled, samples were extracted by the addition of $1 \mathrm{~mL}$ of $0.9 \% \mathrm{NaCl}$ and $2 \mathrm{~mL}$ of $n$-hexane (Fisher Scientific). Following centrifugation at $4000 \mathrm{rpm}$ for 5 minutes, the top layer was transferred to a fresh glass vial and dried under a steady stream of nitrogen. The sample was dissolved in $50 \mu \mathrm{L}$ of $n$-hexane and $2 \mu \mathrm{L}$ of the sample was analyzed on a Varian $450-\mathrm{GC}$ equipped with a HP1 capillary column $(15 \mathrm{~m} \times 250 \mu \mathrm{m}$ i.d., $0.25 \mathrm{um}$ film thickness, Varian, Santa Clara, CA) using helium as the carrier gas at a flow rate of $2 \mathrm{~mL} / \mathrm{min}$. The injector was held at $225^{\circ} \mathrm{C}$ and the oven temperature was held at $100^{\circ} \mathrm{C}$ for 5 minutes and then increased from $100^{\circ} \mathrm{C}$ to $160^{\circ} \mathrm{C}$ at a rate of $20^{\circ} \mathrm{C}$ per minute, followed by an 8 minute hold, and then an increase of $10^{\circ} \mathrm{C}$ per minute to a final temperature of $240^{\circ} \mathrm{C}$. The oven was held at $240^{\circ} \mathrm{C}$ for 5 minutes (method from Mayer and Shanklin, 2007). Results were compared over three biological replicates. 
To analyze the $\mathrm{LB}$ media for the presence of $\beta$-ketoacids, $1 \mathrm{~mL}$ of the media from the pelleted culture was transferred to a fresh solvent-rinsed glass vial. $1 \mathrm{~mL}$ of $2 \mathrm{M}$ sulfuric acid was added to the media and the sample incubated at $75^{\circ} \mathrm{C}$ and subsequently $30^{\circ} \mathrm{C}$ for 30 minutes each. The 3-ketoacids were then extracted with the addition of $1 \mathrm{~mL}$ of $n$-hexane. After centrifugation at $4000 \mathrm{rpm}$ for 10 minutes, $2 \mu \mathrm{L}$ of the hexane were analyzed on a Varian $450-G C$ fitted with a HP-1 capillary column ( $15 \mathrm{~m}$ x $250 \mu \mathrm{m}$ i.d., $0.25 \mathrm{um}$ film thickness, Varian) using helium as the carrier gas at a flow rate of 1.5 $\mathrm{mL} / \mathrm{min}$. The injector temperature was held at $250^{\circ} \mathrm{C}$. The oven temperature was initially $50^{\circ} \mathrm{C}$ and held for 8 minutes, followed by an increase to $275^{\circ} \mathrm{C}$ at a rate of $15^{\circ} \mathrm{C}$ per minute. The oven was held at $275^{\circ} \mathrm{C}$ for 5 minutes (method from Yu et al., 2010). Results were compared over three biological replicates.

\subsection{Western blot analysis}

Western blot analysis was carried out to ensure successful induction of the MEL proteins. Each cell pellet was resuspended in $5 \mathrm{~mL}$ of PBS and the cell suspensions diluted to an optical density of 0.75 at $600 \mathrm{~nm}$. $100 \mu \mathrm{L}$ of cell suspension was added to $25 \mu \mathrm{L}$ of $4 \mathrm{X}$ SDS loading dye and the samples boiled for 10 minutes. Following centrifugation at $14000 \mathrm{rpm}$ for 5 minutes, $20 \mu \mathrm{L}$ aliquots were loaded into a $12 \%$ SDS page polyacrylamide resolving gel with a $4 \%$ SDS page stacking gel. $5 \mu \mathrm{L}$ aliquots were also loaded into a second SDS page gel to be transferred to a nitrocellulose membrane for Western blot analysis. The gel was run for 30 minutes at 100 volts and then at 150 volts for approximately another hour using a Bio-Rad Mini-Protean Tetra Cell system (BioRad, Hercules, CA). The gel loaded with more protein was stained with coomassie stain 
overnight on a rocker table and destained with $25 \%$ methanol. The coomassie gel was imaged with an Alpha Innotech fluorchemQ (Alpha Innotech). The gel loaded with less protein was transferred to a $0.45 \mu \mathrm{m}$ nitrocellulose membrane (Bio-Rad) in a Bio-Rad Mini Trans-Blot Electrophoretic Transfer Cell system (Bio-Rad) at $100 \mathrm{~mA}$ in the cold room overnight.

The Western blot to detect tagged proteins was performed using the Amersham ECL Advance Western Blotting Detection Kit (GE Healthcare, Uppsala SE). The membrane was blocked in 5\% fat-free powdered milk in TRIS-buffered saline (TBS-T; $25 \mathrm{mM}$ TRIS pH 7.6, $0.8 \% \mathrm{NaCl}$, and $0.1 \%$ Tween 20 ), for 1 hour on a rocker table and then incubated for 1 hour with T7 tag monoclonal antibody (Novagen) diluted 1:50,000 in blocking solution. The membrane was rinsed three times with TBS-T and washed for 15 min with TBS-T followed by 3 washes with TBS-T of 5 min each. The membrane was incubated for 1 hour in Amersham ECL anti-mouse IgG, horseradish peroxidase whole antibody (GE Healthcare) diluted 1:50,000 in blocking solution and the washes repeated. The ECL advanced Western blotting detection reagents 1 and 2 were mixed at a 1:1 ratio. The membrane was then incubated in the detection reagent for $3 \mathrm{~min}$ in the dark and the excess detection reagent drained off. The membrane was imaged with an Alpha Innotech FluorchemQ.

\subsection{Enzyme analysis}

His-tagged MEL1 and MEL1(D64A) produced in BL21(DE3) were affinity purified by nickel-agarose chromatography. pET28a-MEL1 and pET28a-MEL1(D64A) in $E$. coli BL21(DE3)pLysS cells were inoculated into $5 \mathrm{~mL}$ of LB with $50 \mu \mathrm{g} / \mathrm{mL}$ of 
each kanamycin and chloramphenicol and grown overnight at $37^{\circ} \mathrm{C}$ in a rotating wheel. A $50 \mathrm{~mL}$ culture of $\mathrm{LB}$ with $50 \mu \mathrm{g} / \mathrm{mL}$ each of kanamycin and chloramphenicol was inoculated with $1 \mathrm{~mL}$ of the overnight culture and grown at $30^{\circ} \mathrm{C}$, with shaking at 200 rpm, overnight. $20 \mathrm{~mL}$ of each $50 \mathrm{~mL}$ culture was used to inoculate $2 \mathrm{~L}$ of LB with 50 $\mu \mathrm{g} / \mathrm{mL}$ of each kanamycin and chloramphenicol. These cultures were grown at $30^{\circ} \mathrm{C}$ until the optical density at $600 \mathrm{~nm}$ reached 0.5 to 0.7 , at which point the cultures were induced with $0.5 \mathrm{mM}$ IPTG and grown overnight at $18^{\circ} \mathrm{C}$ with shaking at $180 \mathrm{rpm}$.

Cells where harvested by centrifugation at $5000 \mathrm{rpm}$ for 15 minutes in a Sorvall RC6+ centrifuge (ThermoScientific, Waltham MA). Cells were then washed with approximately $100 \mathrm{~mL}$ of $0.85 \% \mathrm{NaCl}$ and centrifuged for 15 minutes at $5000 \mathrm{rpm}$ with an Eppendorf centrifuge 5810R and an A4 -6- 2 rotor (Eppendorf, Mississauga ON). The pellets were then resuspended in $80 \mathrm{~mL}$ of lysis buffer comprised of $20 \mathrm{mM}$ sodium phosphate buffer ( $\mathrm{pH} 7.4$ ), 0.5 M NaCl, and $15 \mathrm{mM}$ imidazole. Lysozyme (Invitrogen) was added to each tube of resuspended cells to a final concentration of approximately 1 $\mathrm{mg} / \mathrm{mL}$ and incubated at room temperature for 30 minutes. Cells were then lysed by repeatedly freezing the suspension in liquid nitrogen, allowing it to thaw 3 times, and then sonicated with a sonicator (Sonics and Materials, Newtown CT) for 9 intervals of sonication for 30 seconds with rests in between on ice for 30 seconds. The samples were centrifuged at $15000 \mathrm{rpm}$ for 1 hour at $4^{\circ} \mathrm{C}$ in a Sorvall RC6 + centrifuge in order to pellet the insoluble fraction. The soluble fraction was then filtered through a $0.45 \mu \mathrm{M}$ filter into a clean flask and kept at $4^{\circ} \mathrm{C}$.

The nickel-agarose (Qiagen, Germantown MD) was resuspended in the storage buffer and poured into a Poly-Prep Chromatography Column (Bio-Rad) so that the final 
agarose volume was approximately $0.5 \mathrm{~mL}$. The column was then equilibrated with 30 $\mathrm{mL}$ of lysis buffer. The filtered soluble fraction from the cells was applied to the column and then rinsed with $30 \mathrm{~mL}$ of lysis buffer. The proteins were eluted with a stepwise imidazole gradient of $50 \mathrm{mM}, 100 \mathrm{mM}, 150 \mathrm{mM}$, and $200 \mathrm{mM}$ imidazole. Approximately $10 \mathrm{~mL}$ of each elution buffer, comprised of $20 \mathrm{mM}$ sodium phosphate ( $\mathrm{pH}$ 7.4), $0.5 \mathrm{M} \mathrm{NaCl}$, and varying imidazole concentrations, was added to the column in succession and the elute collected in $1 \mathrm{~mL}$ fractions. Western blot analysis was carried out as described previously to detect which elute fraction contained the highest levels of MEL1 or MEL1(D64A).

Fractions containing the highest amounts of enzyme were pooled and dialyzed against $50 \mathrm{mM}$ potassium phosphate (pH 7.4) with $1 \mathrm{mM}$ EDTA at $4^{\circ} \mathrm{C}$ overnight. Following dialysis, $0.5 \mathrm{~mL}$ of the dialyzed fraction was concentrated using an Amicon Ultra-0.5, Ultracel-3 Membrane, $3 \mathrm{kDa}$ (Millipore, Darmstadt Germany) centrifuged at $14000 \mathrm{~g}$ for $30 \mathrm{~min}$ at $4^{\circ} \mathrm{C}$. Glycerol was added to the concentrated protein to a final concentration of $15 \%$, and then the protein extract was aliquoted and frozen at $-80^{\circ} \mathrm{C}$ until use.

The acyl-CoA thioesterase activity of MEL1 and MEL1 (D64A) was measured using 5,5'-dithiobis-(2-nitrobenzoic acid) (DTNB) (Sigma Aldrich, Oakville ON). DTNB reacts with the sulfhydryl group in free Coenzyme A to form 5-thio-2nitrobenzoate (TNB). TNB absorbs light at $412 \mathrm{~nm}$ with an extinction coefficient of $13600 \mathrm{M}^{-1} \mathrm{~cm}^{-1}$ (Ellman, 1958). The enzymatic reactions were carried out in a 96 well plate (Greiner bio-one, Frickenhausen DE) with $50 \mathrm{mM} \mathrm{KCl}$, and $0.3 \mathrm{mM}$ DTNB as outlined by Wei et al. (2009). To each $200 \mu \mathrm{L}$ reaction, palmitoyl-CoA (Sigma Aldrich, 
Oakville ON) was added to a final concentration of $50 \mu \mathrm{M}$. Reactions were mixed through gentle pipetting and $2 \mu \mathrm{L}$ of concentrated MEL1 enzyme was added to each reaction. Reactions were carried out at $30^{\circ} \mathrm{C}$ with absorbance readings taken at $410 \mathrm{~nm}$ at 10 minute intervals for 10 hours with a FLUOstar OPTIMA microplate reader (BMG Labtech, Offenburg, DE).

Enzymatic reactions can be influenced by many factors including buffer $\mathrm{pH}$ and composition. Reactions were carried out as outlined above with the various buffers: 10 $\mathrm{mM}$ sodium citrate $\mathrm{pH} 5$ and $\mathrm{pH} 6,10 \mathrm{mM}$ sodium phosphate $\mathrm{pH} 6, \mathrm{pH} 7, \mathrm{pH} \mathrm{7.5,} \mathrm{pH} 8$, $10 \mathrm{mM}$ HEPES pH 7, pH 7.5 and $\mathrm{pH} 8$, and 10 mM TRIS pH 8. Differences in activity were determined by using the Beer's-Lambert law to determine the concentration of hydrolyzed CoA present after 150 minutes, a time point during the linear phase of enzymatic activity for all buffers tested.

Based on the above optimization, the MEL1 thioesterase activity was determined in the final mixture: $10 \mathrm{mM}$ sodium phosphate $(\mathrm{pH} 8), 50 \mathrm{mM} \mathrm{KCl}, 0.3 \mathrm{mM}$ DTNB, 50 $\mu \mathrm{M}$ palmitoyl $-\mathrm{CoA}$, and $2 \mu \mathrm{L}$ of enzyme. All reactions were carried out in triplicate. The blank reactions contained dialysis buffer.

2.13 Co-regulation of extracellular lipid biosynthetic genes

The Expression Angler (http://bar.utoronto.ca/) tool provided by the Bio-Array Resource was used to identify thioesterases that may function in extracellular lipid biosynthesis (Toufighi et al., 2005). The cuticle biosynthetic genes listed in Appendix 3 and the suberin biosynthetic genes listed in Appendix 4 were used to search the NASCArrays 392 dataset with an R-value cutoff of 0.5 for correlated genes. The lists of 
correlated genes were then manually mined for genes that correlate with multiple known lipid biosynthetic genes. The microarray data generated by Suh et al. (2005) was used to identify candidate genes expressed in the epidermal layer of stems and gene annotations were used to identify putative thioesterases.

\subsection{Bioinformatics}

Sequences for bioinformatic analysis were aligned with ClustalW (Larkin et al, 2007). Sequences for verified and predicted single Hotdog fold proteins from a variety of plant species, based on the alignments in Yu et al. (2010) and Ben-Israel et al. (2009), were retrieved from NCBI. Catalytic residues were identified by aligning the well characterized 4-HBT protein sequence against the plant sequences (Benning et al., 1998). The tertiary structure of the MEL proteins was predicted using the homology modeling program Swiss-Model, using the Pseudomonas sp. Strain CBS-3 4HBT enzyme as a template (pdb:1LO7_A) (Arnold et al, 2006, Kiefer et al., 2009, Peitsch et al., 1995). Following sequence alignment, neighbor-joining phylogenetic trees were generated with ClustalW (Larkin et al., 2007) to examine the evolutionary relationship between the MEL enzymes. 


\section{Chapter 3: Results}

3.1 Identification of a thioesterase gene family associated with extracellular lipid biosynthesis.

In silico co-regulation analysis was used to identify a novel gene encoding a thioesterase associated with cuticle biosynthesis. Genes that consistently co-expressed with 29 known cuticle biosynthetic genes (Appendix 3) were identified by inspection of 392 publically available DNA microarray experiments, using the Expression Angler tool of the Bio-Array Resource for Plant Biology (Toufighi et al., 2005) (http://bar.utoronto.ca/). Thirty-nine candidate genes annotated as encoding lipid biosynthetic enzymes were identified as significantly co-regulated with 2 or more cuticleassociated genes (Table 2). Of these, 26 were expressed in the epidermal cell layer at levels high enough to be detected above the background noise in the DNA microarray study of Suh et al., 2005, which identified epidermally enriched transcripts in Arabidopsis. Twenty-one of these 26 genes were found to be preferentially expressed at least 2-fold in the epidermal layer compared to underlying tissues either at the top or bottom of the stem (Suh et al., 2005) (Table 2). Members of several families of lipid biosynthetic genes were identified as being co-regulated, such as lipid transfer proteins, $\beta$-ketoacyl-CoA synthases, and fatty acid $\omega$-hydroxylases. Of these co-regulated and epidermally expressed genes, one was annotated as a thioesterase, Atlg35290, which coregulates with 6 cuticle biosynthetic genes (Table 2). We have tentatively named At1g35290 as MODIFIER OF EXTRACELLULAR LIPIDS 1 (MEL1). By using MEL1 as bait in the Expression Angler tool, the reverse analysis was carried out and co-regulated 
genes identified. As expected, the same 6 known cuticle-associated genes were coregulated with a Pearson correlation coefficient greater then 0.5 (Table 3 ). Based on a DNA microarray experiment, $M E L 1$ transcript levels are increased by 3-fold at the stop of the stem, relative to underlying tissues, and 2-fold at the base of the stem (Suh et al., 2005) (Table2).

Three genes related to MEL1 were identified in the Arabidopsis genome: Atlg35250 (MEL2), At1g68260 (MEL3), and At1g68280 (MEL4). The Expression Angler tool was also used to identify co-regulated genes with known suberin-associated genes (Appendix 4). 28 genes were identified that co-regulate with known suberin biosynthetic genes with a Pearson correlation coefficient greater then 0.5 , including MEL2 (Table 4). Using MEL2 as bait in the Expression Angler tool, 7 known suberin associated co-regulated genes were identified (Table 5). While both MEL1 and MEL2 co-regulate with extra-cellular lipid biosynthetic genes, $M E L 3$ gene expression did not obviously correlate with any extracellular lipid biosynthetic genes by inspection of previously published DNA microarray experiments. MEL4 is not present on any current DNA microarrays and therefore co-regulation analysis is not possible. 
Table 2: Genes that are co-expressed with known cuticle biosynthetic genes in DNA microarray experiments.

Candidate cuticle associated genes were identified using the known cuticle genes listed in Appendix 3 as baits in the BAR

Expression Angler tool (Toufighi et al., 2005). Candidate genes were further narrowed based on whether or not they showed

epidermal expression in microarray experiment carried out by Suh et al. (2005). ${ }^{\mathrm{a}}$ The mean fold change in transcript levels found in the epidermis compared to underlying cell layers (Suh et al., 2005).

\begin{tabular}{|c|c|c|c|c|c|}
\hline Annotation & $\begin{array}{c}\text { AGI } \\
\text { number }\end{array}$ & $\begin{array}{c}\text { Number of co- } \\
\text { regulated known } \\
\text { cuticle-associated } \\
\text { genes }\end{array}$ & $\begin{array}{l}\text { Epidermal } \\
\text { expression }\end{array}$ & $\begin{array}{c}\text { Top } \\
\text { epidermal } \\
\text { ratio }^{a}\end{array}$ & $\begin{array}{c}\text { Base } \\
\text { epidermal } \\
\text { ratio }^{\mathrm{a}}\end{array}$ \\
\hline Lipid transfer protein & Atlg62500 & 8 & $\mathrm{Y}$ & 1.5 & 1.2 \\
\hline Lipid transfer protein (LTP6) & At3g08770 & 6 & $\mathrm{Y}$ & 1.2 & 3.9 \\
\hline Lipid transfer protein (LTP5) & At3g51600 & 5 & $\mathrm{Y}$ & 2.8 & 3.6 \\
\hline Lipid transfer protein & At3g43720 & 4 & $\mathrm{Y}$ & 3.2 & 4.7 \\
\hline Lipid transfer protein & At5g62080 & 4 & $\mathrm{Y}$ & 1.6 & 1.2 \\
\hline Lipid transfer protein (LTP4) & At5g59310 & 4 & $\mathrm{Y}$ & 1.5 & 1.4 \\
\hline Lipid binding & Atlg55260 & 3 & $\mathrm{Y}$ & 2.6 & 10.6 \\
\hline Lipid transfer protein (LTP7) & At2g15050 & 2 & $\mathrm{Y}$ & 4.3 & 3.4 \\
\hline Lipid transfer protein & At2g10940 & 2 & $\mathrm{Y}$ & 0.5 & 10.3 \\
\hline Lipid transfer protein (LTP3) & At5g59320 & 2 & Y & 1.6 & 1.5 \\
\hline$\beta$-ketoacyl-CoA synthase (CER60/KCS5) & Atlg25450 & 4 & $\mathrm{Y}$ & 5.8 & 10.9 \\
\hline$\beta$-ketoacyl-CoA synthase (KCS10/FDH) & At2g26250 & 4 & Y & 4 & 3.9 \\
\hline$\beta$-ketoacyl-CoA synthase (KCS3) & Atlg07720 & 4 & Y & 9.9 & 2.7 \\
\hline$\beta$-ketoacyl-CoA synthase (KCS19) & At5g04530 & 2 & $\mathrm{Y}$ & 8.7 & 41.3 \\
\hline Transferase & Atlg65450 & 8 & $\mathrm{Y}$ & 3.8 & 6.5 \\
\hline Acyltransferase (LPAT5) & At3g18850 & 7 & Y & 2.3 & 2.2 \\
\hline
\end{tabular}




\begin{tabular}{|c|c|c|c|c|c|}
\hline Transferase & At3g48720 & 4 & $\mathrm{Y}$ & 4 & 4.8 \\
\hline Oxidoreductase (HOTHEAD) & Atlg72970 & 6 & Y & 4.1 & 4.3 \\
\hline Oxidoreductase (CER1 LIKE) & Atlg02190 & 7 & $\mathrm{Y}$ & 9.8 & 2.6 \\
\hline $\mathrm{ABC}$ transporter (WBC18) & At3g55110 & 9 & $\mathrm{Y}$ & 3 & 2.3 \\
\hline $\mathrm{ABC}$ transporter & Atlg51460 & 6 & $\mathrm{Y}$ & 1 & 0.6 \\
\hline Fatty acid $\omega$-hydroxylase (CYP86A7) & Atlg63710 & 7 & $\mathrm{Y}$ & 2.7 & 6.5 \\
\hline Fatty acid $\omega$-hydroxylase (CYP86A4) & Atlg01600 & 4 & $\mathrm{Y}$ & 2.5 & 18.1 \\
\hline Thioesterase & Atlg35290 & 6 & Y & 3 & 2.2 \\
\hline Fatty acid desaturase & Atlg06350 & 4 & $\mathrm{Y}$ & 3.1 & 41.3 \\
\hline Aldehyde dehydrogenase (ALDH3F1) & At4g36250 & 4 & Y & 4.6 & 6.3 \\
\hline Lipid transfer protein & Atlg66850 & 7 & $\mathrm{~N}$ & N/A & N/A \\
\hline Lipid transfer protein & At5g07230 & 6 & $\mathrm{~N}$ & N/A & N/A \\
\hline Lipid transfer protein & At3g07450 & 2 & $\mathrm{~N}$ & N/A & N/A \\
\hline Lipid transfer protein (LTP12) & At3g51590 & 2 & $\mathrm{~N}$ & N/A & N/A \\
\hline Glycine rich protein 19 & At5g07550 & 5 & $\mathrm{~N}$ & N/A & N/A \\
\hline Glycine rich protein 20 & At5g07560 & 2 & $\mathrm{~N}$ & N/A & N/A \\
\hline Glycerol 3-phosphate acytransferase (GPAT6) & At2g38110 & 6 & $\mathrm{~N}$ & N/A & N/A \\
\hline Transferase & At5g23970 & 2 & $\mathrm{~N}$ & N/A & N/A \\
\hline Short-chain dehydrogenase/reductase (SDR) & At3g55290 & 6 & $\mathrm{~N}$ & N/A & N/A \\
\hline Fatty Acid Reductase (MS2) & At3g11980 & 2 & $\mathrm{~N}$ & N/A & N/A \\
\hline $\mathrm{ABC}$ transporter & At3g13220 & 2 & $\mathrm{~N}$ & N/A & N/A \\
\hline 3-ketoacyl-CoA synthase (KCS13) & At2g46720 & 4 & $\mathrm{~N}$ & N/A & N/A \\
\hline Acyl-CoA synthetase (ACOS5) & Atlg62940 & 2 & $\mathrm{~N}$ & N/A & N/A \\
\hline
\end{tabular}


Table 3: $M E L 1$ co-expresses with known cuticle biosynthetic genes.

$\mathrm{R}^{2}$ is the Pearson correlation coefficient that represents the similarity of the gene expression patterns over 392 DNA microarray experiments. The closer the value is to 1 , the more similar the gene expression pattern in the analyzed DNA microarray experiments (Toufighi et al., 2005).

\begin{tabular}{ccc} 
AGI number & Gene Name & $\mathbf{R}^{2}$ \\
\hline At1g35290 & MEL1 & 1.00 \\
At1g57750 & $M A H 1$ & 0.712 \\
At4g33790 & CER4 & 0.643 \\
At5g25390 & SHN2 & 0.595 \\
At5g37300 & WSD1 & 0.587 \\
At1g02205 & CER1 & 0.545 \\
At5g57800 & CER3 & 0.517 \\
At2g47240 & CER8 & 0.512
\end{tabular}


Table 4: Genes that are co-expressed with known suberin biosynthetic genes in DNA

microarray experiments.

Candidate suberin associated genes were identified using the known suberin associated genes listed in Appendix 4 as baits in the BAR Expression Angler tool (Toufighi et al., 2005).

Number of co-
regulated known
suberin-associated Annotation AGI number genes

Lipid transfer protein

Lipid transfer protein

At5g13900 6

Lipid transfer protein

At2g18370

6

Lipid transfer protein

At3g58550

5

Lipid transfer protein

At2g48130

5

Lipid transfer protein

At3g22620

5

Lipid transfer protein

At3g53980

5

Lipid transfer protein

At4g22610

5

Lipid transfer protein

At5g05960

4

At5g46890

4

Lipid transfer protein related

At2g44300

4

Lipid transfer protein

At2g37870

3

Transferase

At 1 g78990

\section{6}

Transferase

At5g07870

Transferase

At5g63560

5

Transferase

At4g31910

$\mathrm{ABC}$ transporter

At5g13580

7

$A B C$ transporter

At3g53510

6

$A B C$ transporter

Atlg53270

\section{5}

$\mathrm{ABC}$ transporter

At2g37360

4

$\mathrm{ABC}$ transporter

At5g19410

2

Thioesterase

Atlg35250

FATA

At3g25110

2

Fatty acid desaturase

At1g06090

\section{3}

Short-chain dehydrogenase/reductase (SDR)

Alcohol dehydrogenase

At1g64590

\section{6}

At1g22440 4

Short-chain dehydrogenase/reductase (SDR)

At4g13180

Short-chain dehydrogenase/reductase (SDR)

At3g26760

2

Lipid binding

At4g33550

6 
Table 5: MEL2 co-expresses with known suberin biosynthesis genes.

$\mathrm{R}^{2}$ is the Pearson correlation coefficient that represents the similarity of the gene expression patterns over 392 DNA microarray experiments. The closer the value is to 1 , the more similar the gene expression pattern in the analyzed DNA microarray experiments (Toufighi et al., 2005).

\begin{tabular}{ccc} 
AGI number & Gene Name & $\mathbf{R}^{\mathbf{2}}$ \\
\hline At1g35250 & MEL2 & 1.00 \\
At3g44540 & FAR4 & 0.74 \\
At5g58860 & CYP86A1 & 0.715 \\
At5g23190 & CYP86B1 & 0.709 \\
At5g41040 & AFST & 0.657 \\
At2g11430 & GPAT5 & 0.607 \\
At3g44550 & FAR5 & 0.502
\end{tabular}


3.2 The predicted MEL proteins are Hotdog fold thioesterases

The predicted MEL proteins are relatively small, about 180 amino acids in length with a molecular weight of about $20 \mathrm{kDa}$. The MEL proteins share greater then $80 \%$ similarity with one another at the amino acid level (Figure 10A and B). The conserved domain database from NCBI identifies a single Hotdog fold domain within each of the MEL proteins, related to the 4HBT Hotdog fold subfamily (Figure 10C) (Marchler-Bauer et al., 2009). All MEL proteins contain the conserved catalytic aspartate, position 64 in MEL1, identified in the bacterial 4HBT thioesterase, and two other conserved residues, a glycine at position 67 and a valine at position 69 in MEL1, which contribute to the binding pocket of the 4HBT thioesterase (Figure 10A). Many bacterial members of the 4HBT subfamily contain a conserved tyrosine residue at position 24 in $4 \mathrm{HBT}$, which is predicted to function in nucleophilic attack (Thoden et al., 2003), but this residue not conserved in the MELs. However a conserved tyrosine at position 27 in 4HBT may fulfill this role (Figure 10A; Appendix 5). These proteins are not only similar to the wellcharacterized 4HBT bacterial thioesterase (Benning et al., 1998), but to several thioesterases from other plant species, including the recently characterized METHYLKETONE SYNTHASE 2 (MKS2) protein from Solanum Habrochaites glabratum, a species of tomato (Yu et al., 2010) (Appendix 5). 
Figure 10: The MEL protein family shares a high level of similarity and contains a single characteristic Hotdog fold domain.

The structure of the Hotdog fold is annotated on the alignment ( $\alpha$-helix $(\alpha)$ and $\beta$-sheet $(\beta))$. The catalytic aspartic acid mutated in the MEL1(D64A) mutant is indicated with an asterisk (A). The phylogenetic tree indicates the similarity between MEL1 and MEL2 and MEL3 and MEL4, suggesting a possible gene duplication event. Distances are indicated on the tree (B). A three dimensional representation of the MEL1 protein sequence modeled on the Pseudomonas 4-HBT backbone shows the characteristic Hotdog fold architecture using SWISS-MODEL (http://swissmodel.expasy.org/) (C). Accession numbers: Atlg35290, NP 564457; Atlg35250, NP_174759; Atlg68260, NP_564926; At1g68280, NP_176995; 4HBT, 1LO7_A. 
A)

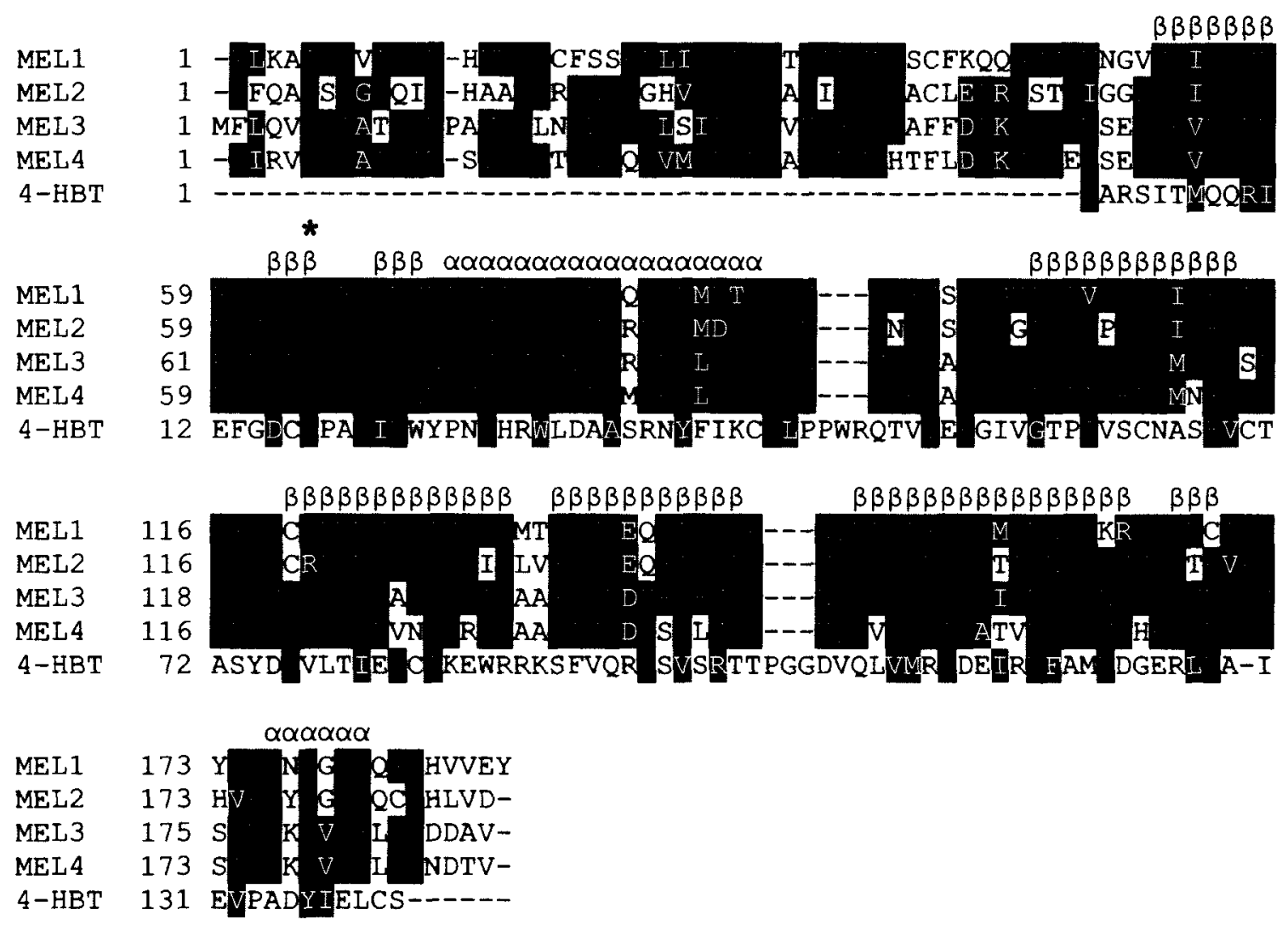

B)

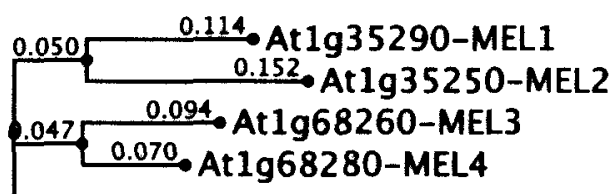

0.350

C)

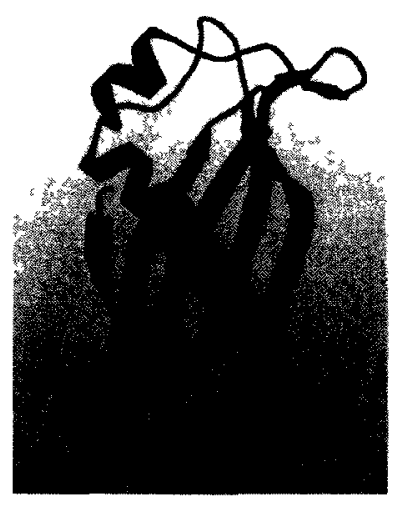


3.3 The $M E L$ family members have distinct gene expression patterns

The tissue-specific gene expression patterns of the $M E L$ genes were first determined by RT-PCR with RNA derived from the roots of 15 day-old seedlings, as well as stems, rosette leaves, and flowers of 6-week-old wild-type plants (Figure 11). MELI expression was highest in stems and flowers, while $M E L 2$ was strongly expressed in roots. MEL3 was expressed constitutively in all tissues tested. The gene expression patterns observed for $M E L 1, M E L 2$, and $M E L 3$ are in agreement with previously published DNA microarray data (Schmid et al., 2005; Winter et al., 2007). RT-PCR was also carried out for MEL4, which showed low levels of flower specific expression. However, upon sequencing of the cloned amplified products it was revealed to be $M E L 3$ transcript, indicating non-specific binding of the MEL4 RT primers.

The gene expression patterns of the $M E L$ family were further investigated by fusing approximately $1800 \mathrm{bp}$ of the genomic sequence upstream of the start codon of each gene in frame with the GUS reporter gene. Following transformation of wild-type Arabidopsis, GUS staining was carried out on at least 10 individual transformants in the first generation and repeated with several lines in the second generation. The promoter of MEL1 drove the expression of GUS in the stem, flower, and siliques of Arabidopsis, which is consistent with the RT-PCR results (Figure 12A). Within the stem, GUS expression was specifically localized to the epidermal cell layer. Weak, but consistent, GUS expression driven by the MEL1 promoter was also seen in the leaf (Figure 12A). This expression however was not detected by RT-PCR. MEL1 is primarily expressed in the epidermis and therefore the transcript may be diluted by sub-surface cells in the whole leaf RNA extraction (Figure 12A) (Rowland et al., 2006). GUS expression driven 
by the MEL2 promoter was found in the endodermal cell layer of the root (Figure 12B). The MEL4 promoter drove GUS expression within the tapetal cells in flower anthers (Figure 12C). Expression of MEL3 was also monitored with GUS expression. However, the expression pattern observed did not match the RT-PCR results and no consistent staining pattern was observed between individual lines. This indicates that the expression observed by GUS analysis was not representative of the native transcript.

To confirm MEL1 epidermal-specific gene expression, an in situ hybridization experiment was carried out. This helped to ensure the expression patterns observed with the MEL1 promoter GUS were representative of the endogenous transcript. A digoxygenin labeled antisense RNA probe was synthesized from the cDNA coding sequence in order to detect which cells in a stem cross section express MEL1. MEL1 transcript was found exclusively in the epidermal cell layer of the stem (Figure 13). This supports the GUS expression, and is also consistent with previously published DNA microarray data (Table 2) (Suh et al, 2005). 


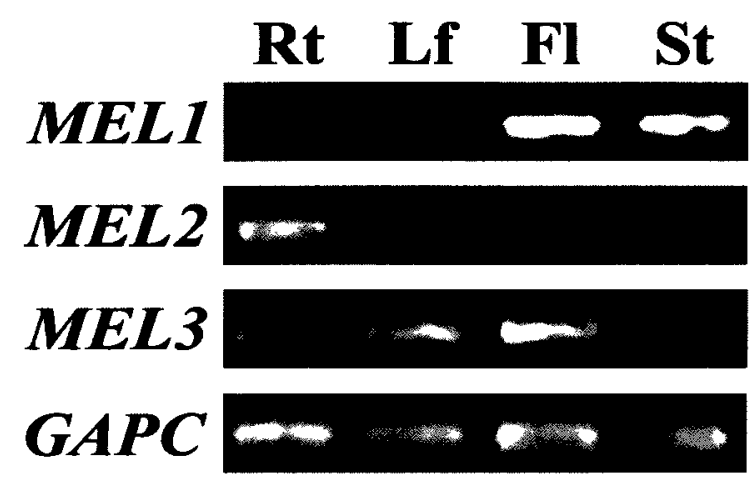

Figure 11: RT-PCR for the tissue specific expression patterns of the $M E L$ gene family. RT-PCR to test for transcript was carried out in roots (Rt), leaf (Lf), flower (Fl), and stem (St). GAPC was used as a constitutively expressed loading control. 


\section{A. MEL1}
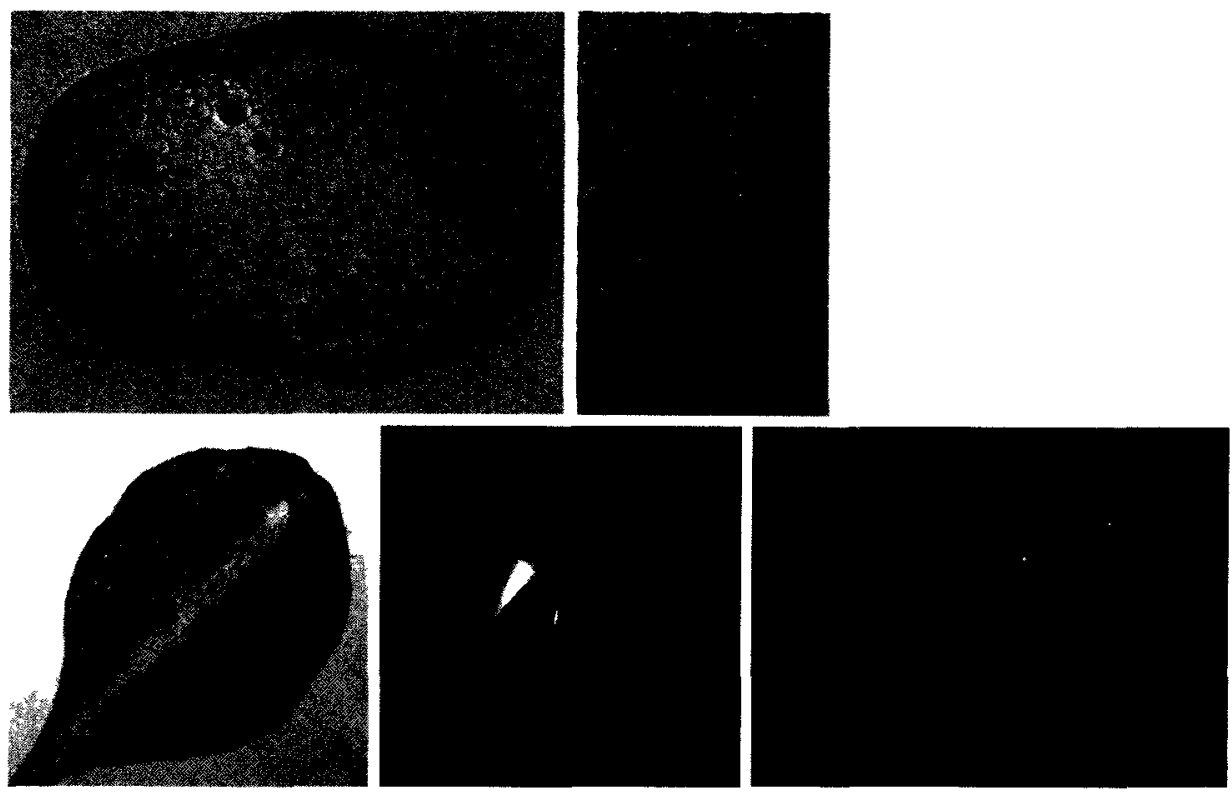

\section{B. MEL2}
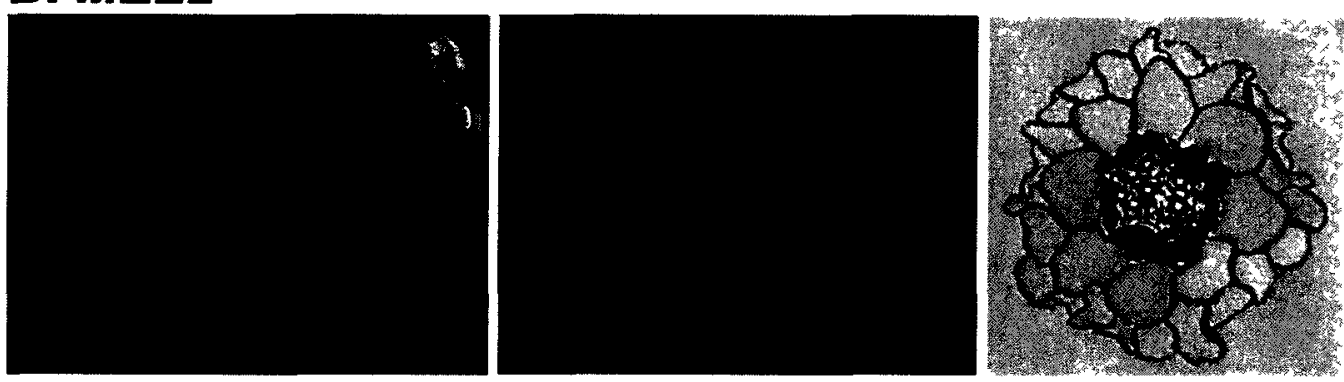

\section{MEL4}
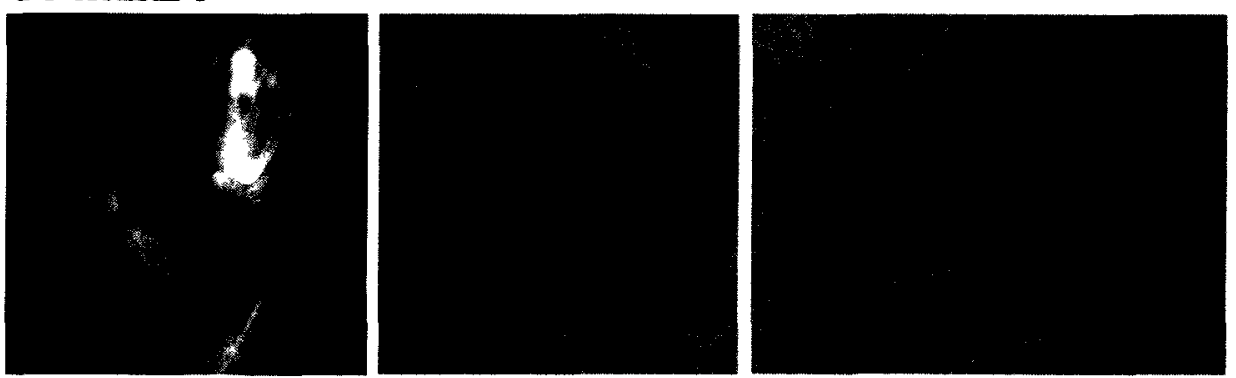

Figure 12: Expression of the GUS reporter gene driven by the $M E L$ promoters.

$M E L 1$ expression was found in stem, leaf, flower, and silique (A), MEL2 expression was found in the root, specifically in the endodermal layer $(\mathbf{B})$, and MEL4 expression was found in tapetal cells of the anther $(\mathbf{C})$. 

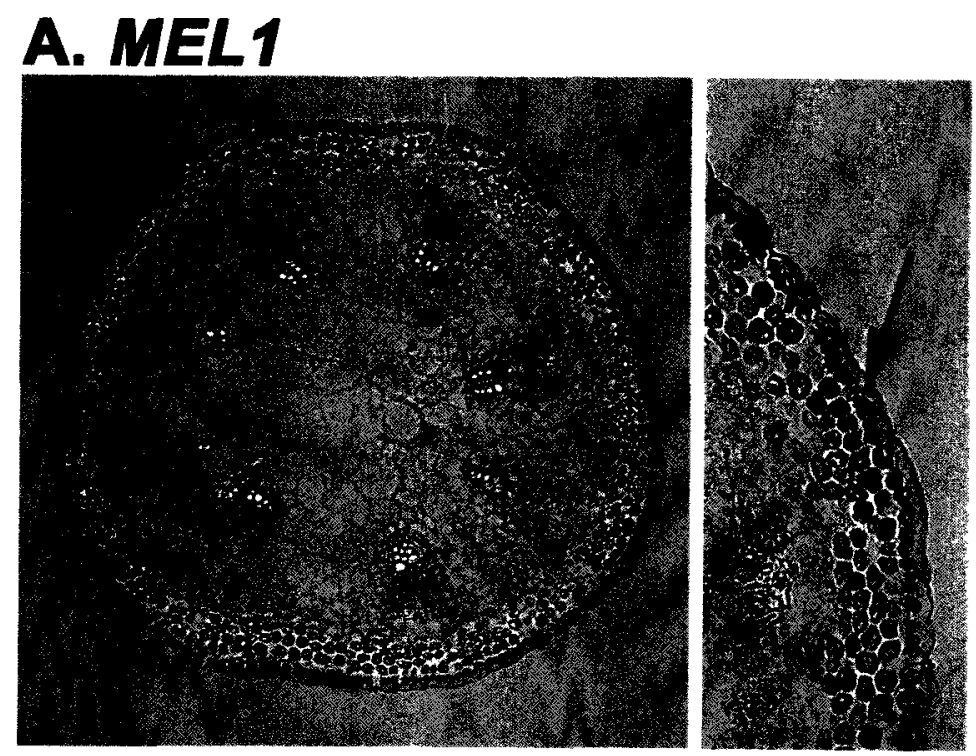

\section{B. CER6}
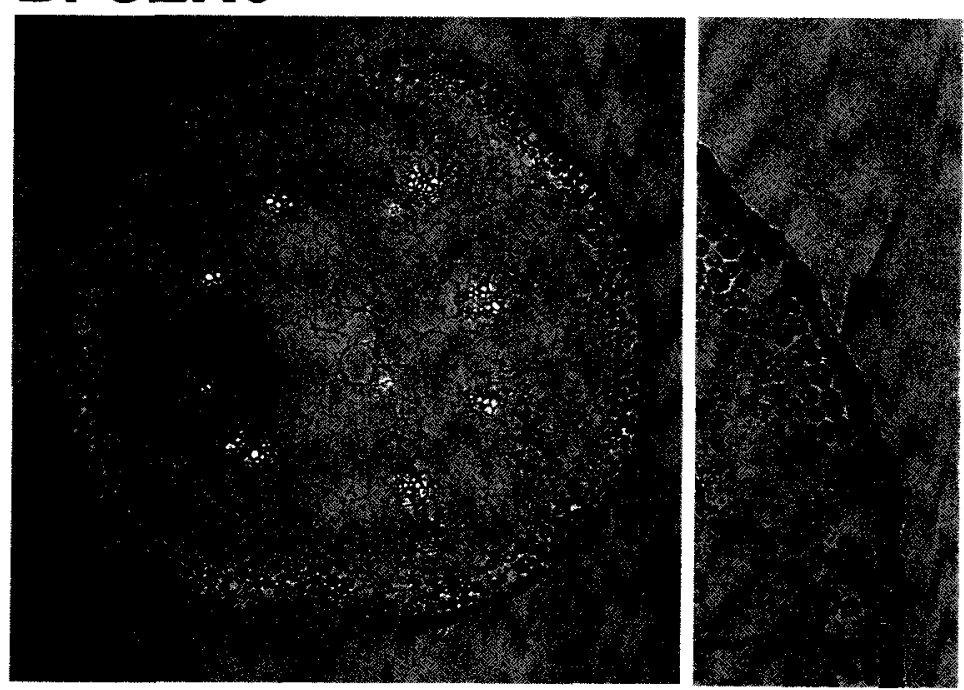

Figure 13: $M E L 1$ epidermal-specific expression as determined by in situ hybridization.

Hybridization was carried out with a digoxygen labeled MELI RNA antisense probe (A). CER6 expression, which is epidermal specific, was used as a positive control (Hooker et al., 2002) (B). 
3.4 Silencing and over-expression of MELI in Arabidopsis

There were no T-DNA or transposon insertion lines available for any of the $M E L$ family members and therefore we were unable to obtain knock-out mutants for these genes commercially. To overcome this, artificial microRNAs (amiRNAs) were employed to decrease the $M E L I$ transcript levels. Two separate amiRNA sequences (A and B) specifically targeting the $M E L 1$ transcript were cloned into binary vectors, downstream of the cauliflower mosaic virus $35 \mathrm{~S}$ promoter, and transformed into Arabidopsis (Figure 14). The amiRNA sequences were cloned into both pBAR::35S and pVKH18::35S to maximize the odds that a successful knockdown line could be achieved. The pBAR::35S contains the core $35 \mathrm{~S}$ promoter with one enhancer sequence and pVKH18::35S contains the core 35 S promoter with six enhancer sequences. Within the first generation of transgenic plants, 5 of the 12 lines examined were identified by RTPCR with decreased MEL1 transcript in the flowers (Figure 15A and B). The numbering system for these five lines is found in Figure 15A. The progeny from the MEL1 amiRNA-2 and MEL1 amiRNA-5 lines, expressing amiRNA sequence $\mathrm{A}$ and $\mathrm{B}$, respectively, were first analyzed in the second generation for alterations in the cuticular wax composition. In spite of decreased transcript in the flowers of the first generation, progeny from $M E L 1$ amiRNA-2 and MEL1 amiRNA-5 showed varying transcript levels in the stem between individual plants in the T2 generation (Figure 16A). This is an indication that the MELI amiRNA-2 and MEL1 amiRNA-5 lines, which were generated using amiRNA sequences cloned into the pBAR::35S vector backbone, likely have unstable expression of the transgene. Nonetheless, the cuticular wax composition of MEL1 amiRNA-2 and MEL1 amiRNA-5 stems was analyzed by GC-FID. In comparison 
to wild-type plants, the cuticular wax composition of MEL1 amiRNA-2 and MEL1 amiRNA-5 was not significantly changed (Figure 16C). This is in agreement with observations in the first generation, as no dramatic changes in cuticular wax composition were observed in that generation either (Appendix 6). However, the inconsistent silencing of the lines makes it difficult to make conclusions about the effects on wax load. Following the analysis of the cuticular wax composition of these plant lines, all subsequent analysis was carried out with MEL1 amiRNA-1 and MEL1 amiRNA-4 lines, which were generated using amiRNA sequences cloned into the pVKH18::35S vector in order to perhaps obtain consistently silenced lines.

$M E L 1$ over-expression lines were also developed to determine if $M E L 1$ functions in cuticle biogenesis. Over-expression constructs were generated by cloning the MELI cDNA sequence downstream of the $35 \mathrm{~S}$ promoter in both the pBAR::35S and pVKH18::35S vectors. Following Agrobacterium-mediated transformation of Arabidopsis, transformants were screened by RT-PCR to identify transformants with increased MEL1 transcript. Of 10 lines examined in the first generation, only 1 was identified with dramatically increased MEL1 transcript levels: pBAR::35S-MEL1(54).11, which from herein is termed MEL1-35S. In the second generation, all plants examined by RT-PCR showed increased $M E L 1$ transcript levels in the stem (Figure 16B). Stems from individuals in the second generation were examined by GC-FID to determine if over-expression of MELI influenced cuticular waxes. Similar to the results seen with MEL1 amiRNA lines, there was no obvious cuticular wax composition changes in the over-expression line when compared to wild-type (Figure 16C). 
The cutin composition of MEL1 amiRNA lines was examined by GC-MS to observe if decreasing $M E L 1$ expression would influence the cutin composition of the leaves or stems. Due to the inconsistent silencing observed in MEL1 amiRNA-2 and MEL1 amiRNA-5 lines, MEL1 amiRNA-1 and MEL1 amiRNA-4 lines were used for cutin analysis. The transcript levels of MELI in the progeny of MELI amiRNA-1 and MELI amiRNA-4 in the flowers were verified by RT-PCR and found to be nearly undetectable in 3 of $6 M E L 1$ amiRNA-1 and 3 of 3 MEL1 amiRNA-4 T2 plants (Figure 17). The cutin monomer composition in the stem or leaf was not significantly affected in the silenced lines in comparison to wild-type (Figure 18). 
Figure 14: The MEL1_amiRNA-A and MEL1_amiRNA-B sequences aligned with the coding sequence from the various $M E L$ genes.

Ideal amiRNA sequences have no mismatches in positions $1-9$ and 12 , and only have 1 or 2 mismatches in positions $13-21$. The less mismatches that are present means the higher the specificity the amiRNA will have for the target (Ossowski et al., 2008). Cross-specificity of MEL1_amiRNA-A with MEL3 is unlikely due to the 2 mismatches in the 5' end of the amiRNA. Similarly, 3 mismatches are present in the 5' region between MEL1_amiRNA-A and $M E L 2$ as well as $M E L 3$. There is a possibility for cross hybridization of MEL1_amiRNA-B with MEL4 as there are no mismatches between MEL1_amiRNA-B and MEL4 in the 5' end and only 2 mismatches in the 3' end. The tissue specificity, however, makes pleiotrophic effects unlikely. Between MEL1_amiRNA-B and $M E L 3$ and $M E L 2$, cross hybridization is unlikely as $M E L 3$ contains a mismatch in the $5^{\prime}$ 'region and MEL2 contains 2 mismatches in the 5 ' region. 


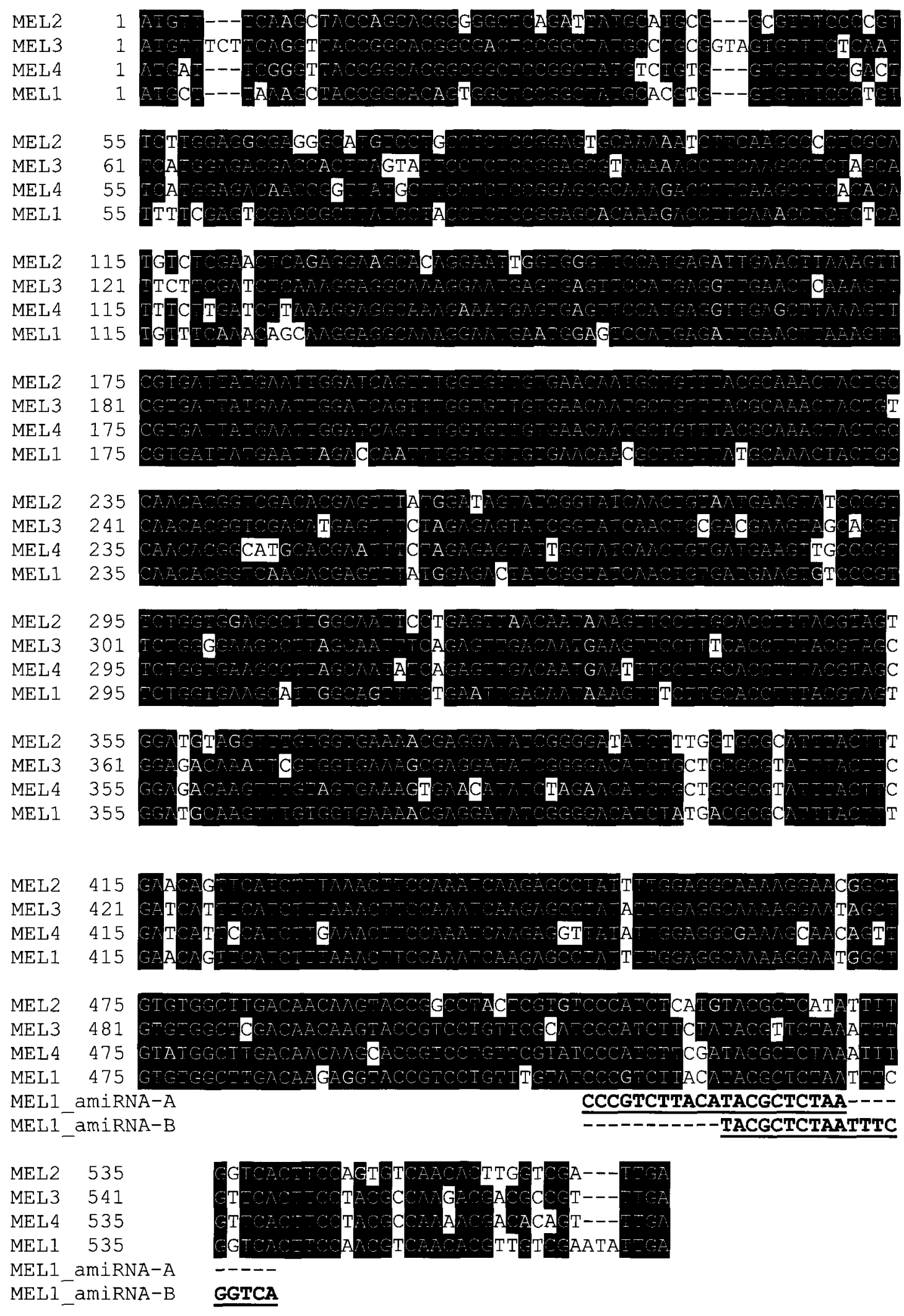


A)

\begin{tabular}{|l|l|}
\hline \multicolumn{1}{|c|}{ Revised plant line nomenclature } & Vector backbone and amiRNA sequence \\
\hline$M E L 1$ amiRNA-1 & pVKH18::35S-MEL1_amiRNA-B \\
\hline$M E L 1$ amiRNA-2 & pBAR::35S-MEL1_amiRNA-A \\
\hline$M E L 1$ amiRNA-3 & pBAR::35S-MEL1_amiRNA-B \\
\hline$M E L 1$ amiRNA-4 & pVKH18::35S-MEL1_amiRNA-A \\
\hline$M E L 1$ amiRNA-5 & pBAR::35S-MEL1_amiRNA-B \\
\hline
\end{tabular}

B)

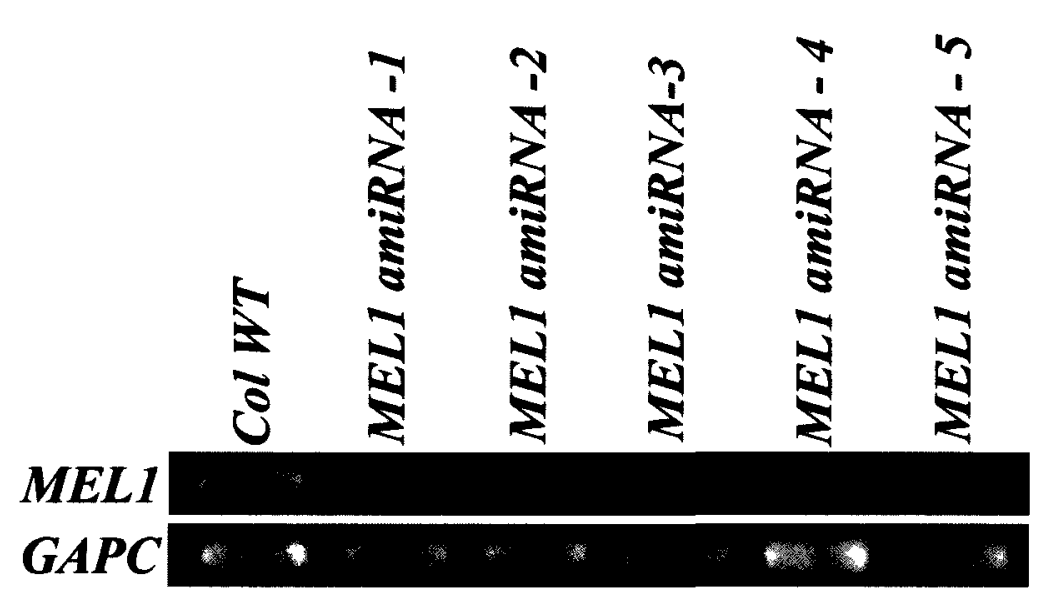

Figure 15: AmiRNA lines were developed to decrease $M E L 1$ transcript levels.

Artificial microRNAs are synthesized by overlapping PCR to replace the naturally occurring Arabidopsis miR319a microRNA with artificial microRNAs directed against $M E L 1$ in the pre-miR319a backbone. Residues were selectively mutated to generate microRNAs specific to the MEL1 sequence. (A) The nomenclature used to describe the individual plant lines in the text is outlined. (B) Several lines in the first generation were identified by RT-PCR with decreased MEL1 transcript in flowers. GAPC was used as a constitutively expressed loading control in panel B. 
Figure 16: Altering the transcript level of $M E L 1$ does not appear to affect the cuticular wax composition.

The lines MEL1 amiRNA-5 and MEL1 amiRNA-2 did not show consistent silencing in stem tissue (A). The MEL1-35S over-expression line identified in the first generation with increased $M E L 1$ transcript levels in stem tissue has consistently increased transcript in the second generation (B). GAPC was used as a constitutively expressed loading control for panels A and B. The levels of cuticular wax was quantified by GC-FID using methyl heptadecanoate as an internal standard and reported as $\mu \mathrm{g} / \mathrm{dm}^{2}(\mathrm{n}=4)(\mathrm{C})$. Progeny from the MEL1 amiRNA-5, MEL1 amiRNA-2, and MEL1-35S lines were used either for RT-PCR or for cuticular wax analysis. ALK, alkane; ALD, aldehyde; FFA, free fatty acid; 1-OH, primary alcohol; KET, ketone; 2-OH, secondary alcohol. 
A)

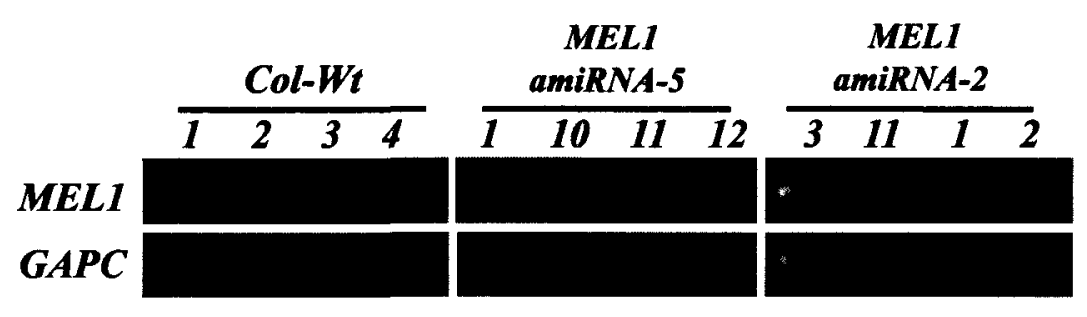

B)

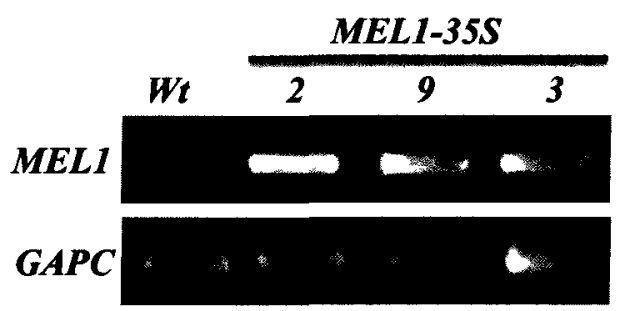

C)

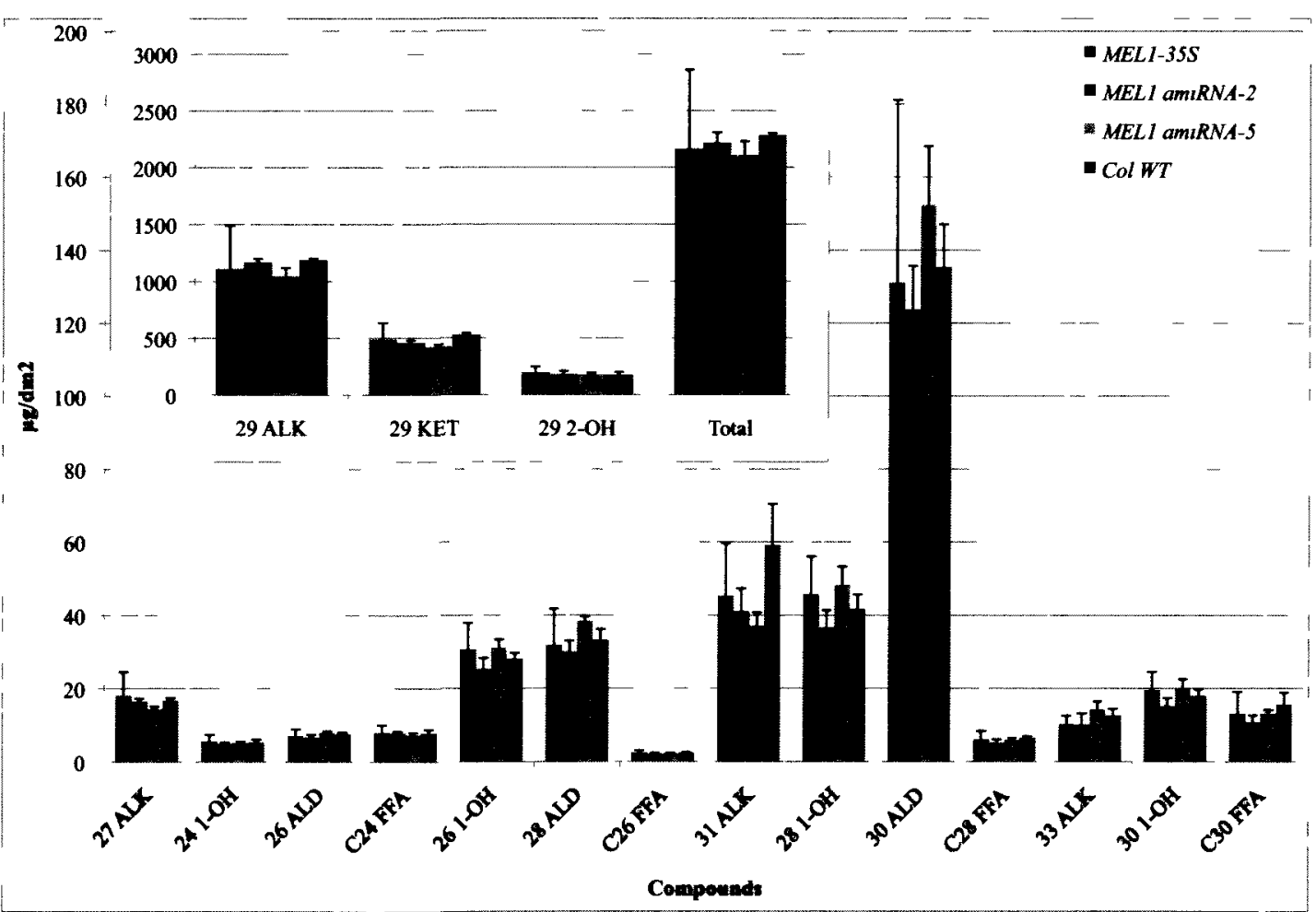




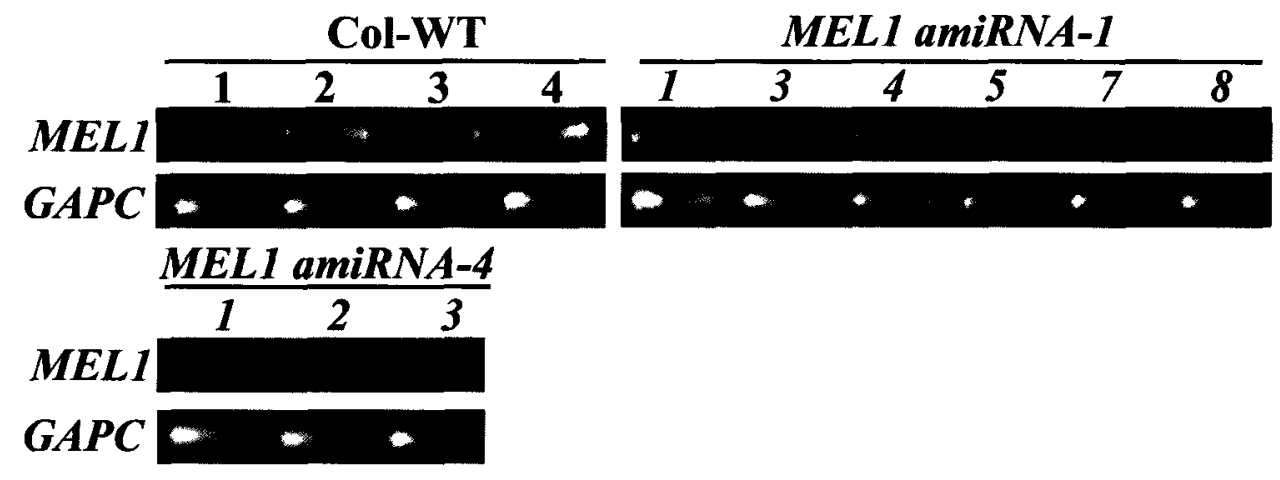

Figure 17: MEL1 amiRNA lines used for cutin composition analysis.

In the second generation, several plants were identified with having decreased MEL1 transcript in flowers. GAPC was used as a constitutively expressed loading control. Cutin analysis was carried out on leaf and stem samples from the same T2 plants: MEL1 amiRNA1-3, MEL1 amiRNA1-7, MEL1 amiRNA1-8 and all three of the MEL1 amiRNA4 progeny (Figure 18). 
Figure 18: The cutin composition of leaf (A) and stem (B) samples from MELI amiRNA1 and MELI amiRNA-4 lines.

Following delipidation, samples were sent to Dr. Frédéric Domergue, CNRS, Laboratory of Membrane Biogenesis, Bordeaux, France. The levels of various cutin-associated compounds were quantified by GC-MS and reported as $\mu \mathrm{g} / \mathrm{mg}$ dry weight (DW) $(n=3)$. $\mathrm{OH}$, Alcohol; FFA, Free fatty acids; DCA, Dicarboxylic acid; wOH, $\omega$-hydroxy fatty acid; diOH, 9,10 dihydroxy fatty acid; 2OH, 2-hydroxy fatty acid. 


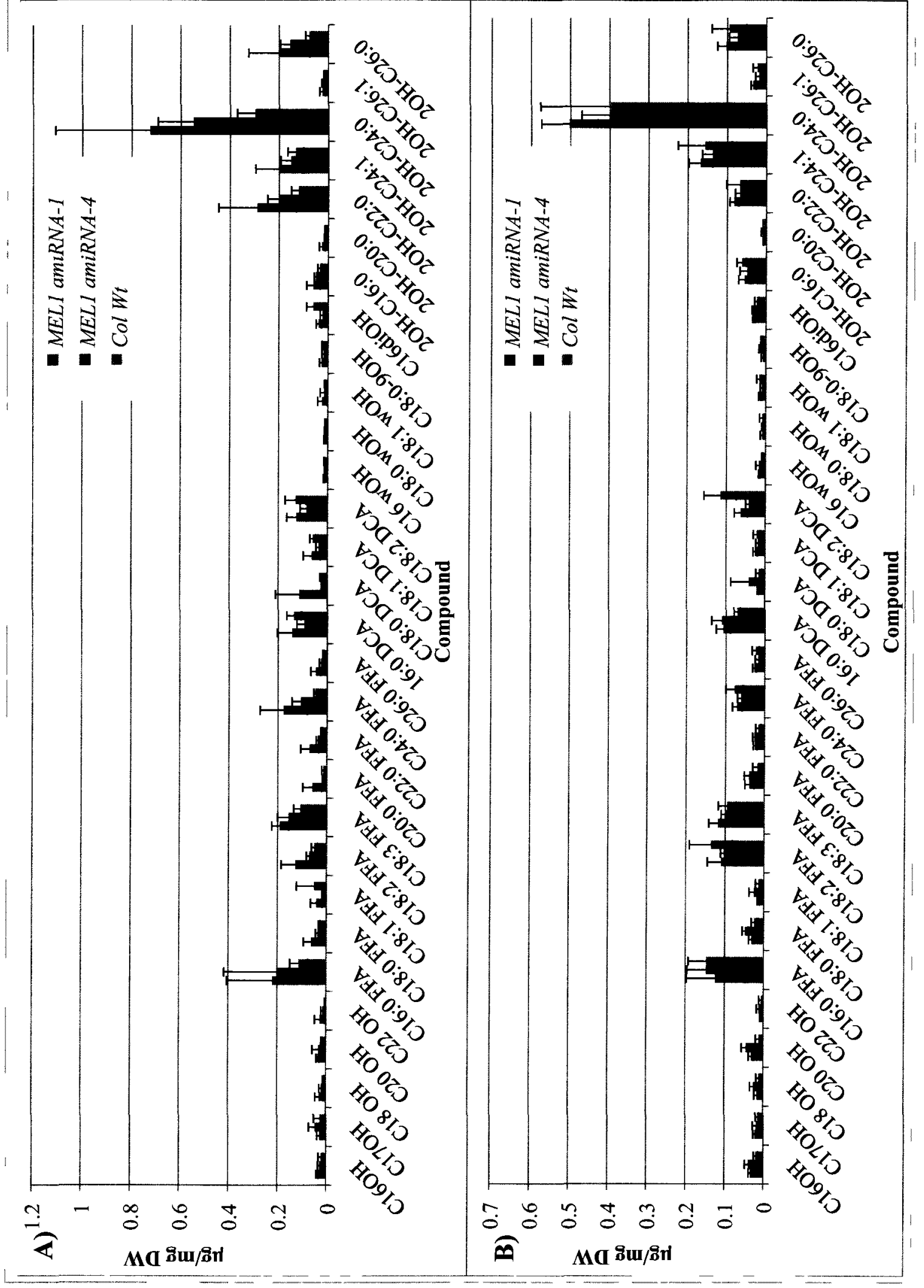


3.5 MEL1 has thioesterase activity in vitro

To examine the possibility that MEL1 has thioesterase activity in vitro, MEL1 was heterologously expressed as a fusion with a T7 epitope and Hisx6 tag. A key catalytic residue required for thioester bond cleavage has been identified in the related proteins 4HBT from Pseudomonas and MKS2 from S. habrochaites (Benning et al., 1998; Yu et al., 2010). This aspartate residue is not only 100\% conserved in the MEL proteins, but also among 15 other uncharacterized single domain Hotdog fold proteins found in plant (Appendix 5). As a control to confirm that MEL1 is carrying out any measured hydrolytic activity, the catalytic aspartate codon at position 64 in $M E L 1$ was mutated to an alanine and the $M E L 1(D 64 A)$ coding region cloned into the $E$. coli expression vector as was done with wild-type MEL1. The MEL proteins were induced in BL21(DE3)pLysS E. coli cells. For comparison, the MEL2 and MEL3 coding sequences were also expressed in E. coli. A cDNA for MEL4 was not successfully isolated. None of the MEL proteins expressed to high levels in BL21 cells, but the expression of the tagged proteins was detectable by Western blot analysis (Figure 19).

The MEL1 and MEL(D64A) proteins produced in BL21(DE3)pLysS were purified using a nickel agarose column. Neither of the proteins was able to be purified to high concentrations, even after centrifugal membrane filtration. It appears that MEL1 purified to near homogeneity, but the MEL1(D64A) extract had a contaminant band at approximately $46 \mathrm{kDa}$ (Figure 20). Following purification, the MEL1 and MEL1(D64A) enzymes were used to test for thioesterase activity with palmitoyl-CoA (C16:0-CoA) substrate. 
Using the general reaction conditions outlined in Wei et al (2009), an optimal buffer composition and $\mathrm{pH}$ was found. The optimal buffer was found to be phosphate buffer (pH 8) (Figure 21). In phosphate buffer (pH 8) MEL1 hydrolyzed palmitoyl-CoA at a rate of approximately $0.09 \mu \mathrm{mol} / \mathrm{min}$, which is approximately $0.01 \mu \mathrm{mol} / \mathrm{min}$ higher then in HEPES ( $\mathrm{pH} 7.5$ ), the second most active buffer. Based on this, the final enzyme reaction contained $10 \mathrm{mM}$ sodium phosphate $\mathrm{pH} 8,50 \mathrm{mM} \mathrm{KCl}, 0.3 \mathrm{mM}$ DTNB, $50 \mu \mathrm{M}$ palmitoyl-CoA, and $2 \mu \mathrm{L}$ of each enzyme. Purified MEL1 was able to hydrolyze approximately $75 \%$ of the palmitoyl-CoA available as the concentration of hydrolyzed palmitoyl-CoA reached a maximum of $38 \mu \mathrm{M}$. MEL1(D64A) enzyme only hydrolyzed $12 \%$ of available substrate indicating that the MEL1 enzyme has significant acyl-CoA thioesterase activity (Figure 22A). The activity of MEL1 toward palmitoyl-CoA was $0.082 \pm 0.028 \mu \mathrm{mol} / \mathrm{min}$ while the activity of MEL1(D64A) was $0.021 \pm 0.014 \mu \mathrm{mol} / \mathrm{min}$ after 150 minutes, which is statistically significant based on a students T-test $(p=0.007$, $\alpha=0.05$ ) (Figure 22B). 
A)

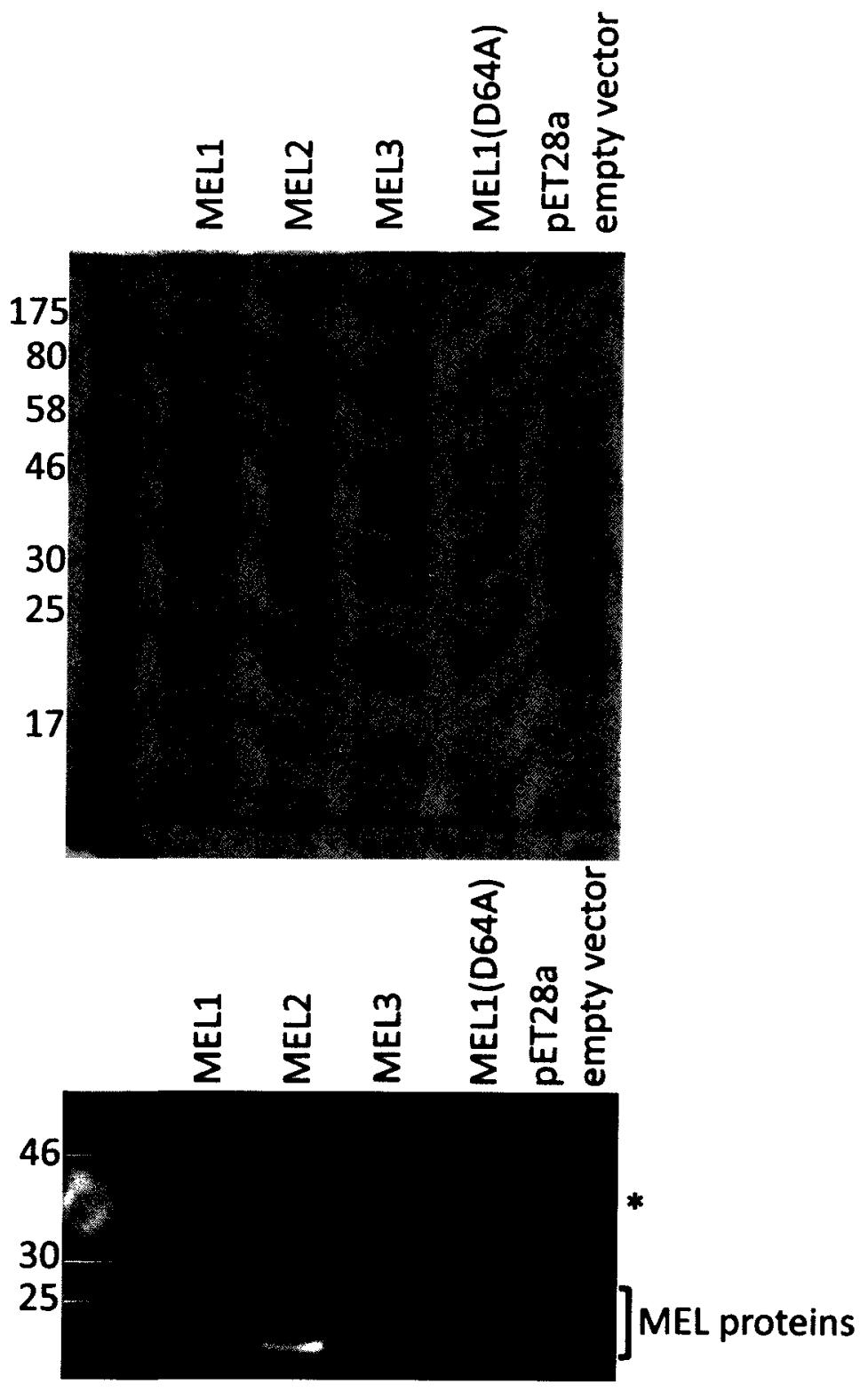

Figure 19: The MEL proteins expressed in BL21(DE3)pLysS E. coli cells.

Crude protein fractions MEL1, MEL2, MEL3, MEL1 (D64A) and empty pET28a vector control run on a $12 \%$ SDS-PAGE gel stained with Coomassie Blue (A). Western blot analysis was performed on the same crude protein fractions using an antibody for the T7 epitope tag at the amino terminus (B). * indicates a non-specific band found in all samples, including empty vector controls. 
A)

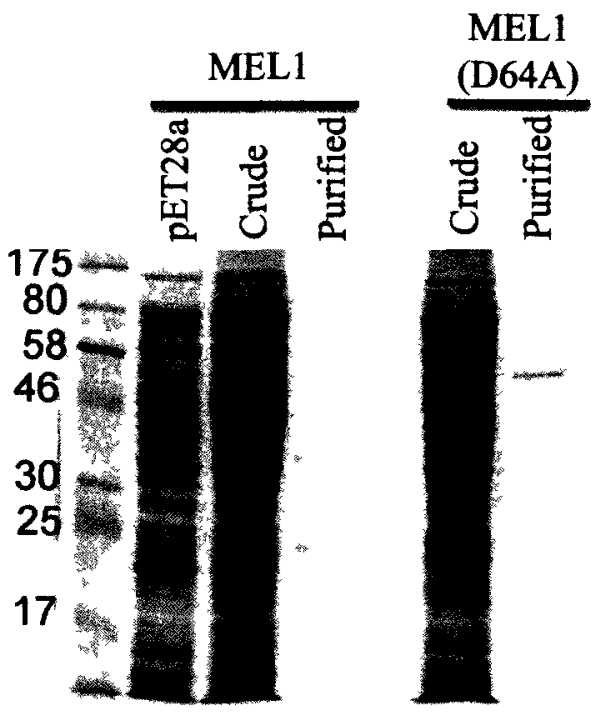

B)

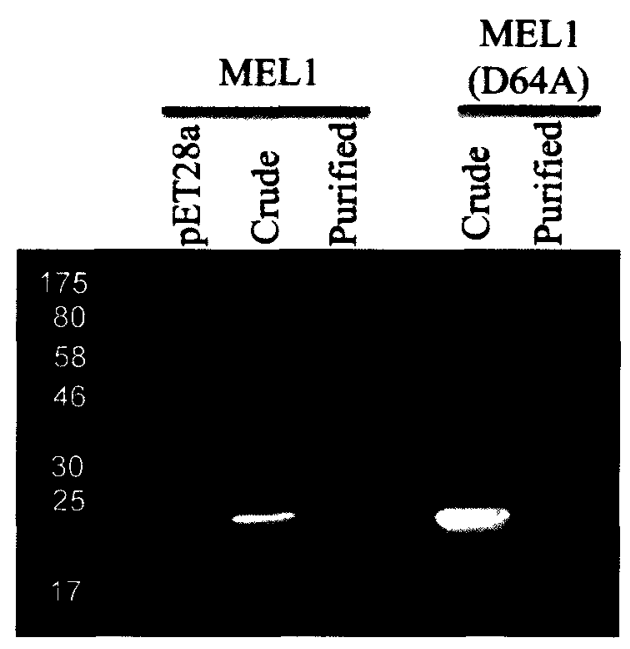

Figure 20: Purification of MEL1 and MEL1(D64A)

The proteins were purified by nickel agarose chromatography and concentrated with an Amicon Ultra-15 centrifuge filter. Protein from concentrated MEL1 and MEL1(D64A) fractions were run on a $12 \%$ SDS-PAGE gel with crude fractions from induced MEL1, MEL1(D64A) and pET28a empty vector and stained with Coomassie blue. The expected size of MEL1 and MEL1(D64A) was approximately $23 \mathrm{kDa}(\mathrm{A})$. Western blot analysis was performed on the protein fractions used for Coomassie stain with an antibody for the $\mathrm{T} 7 \mathrm{tag}(\mathrm{B})$. 

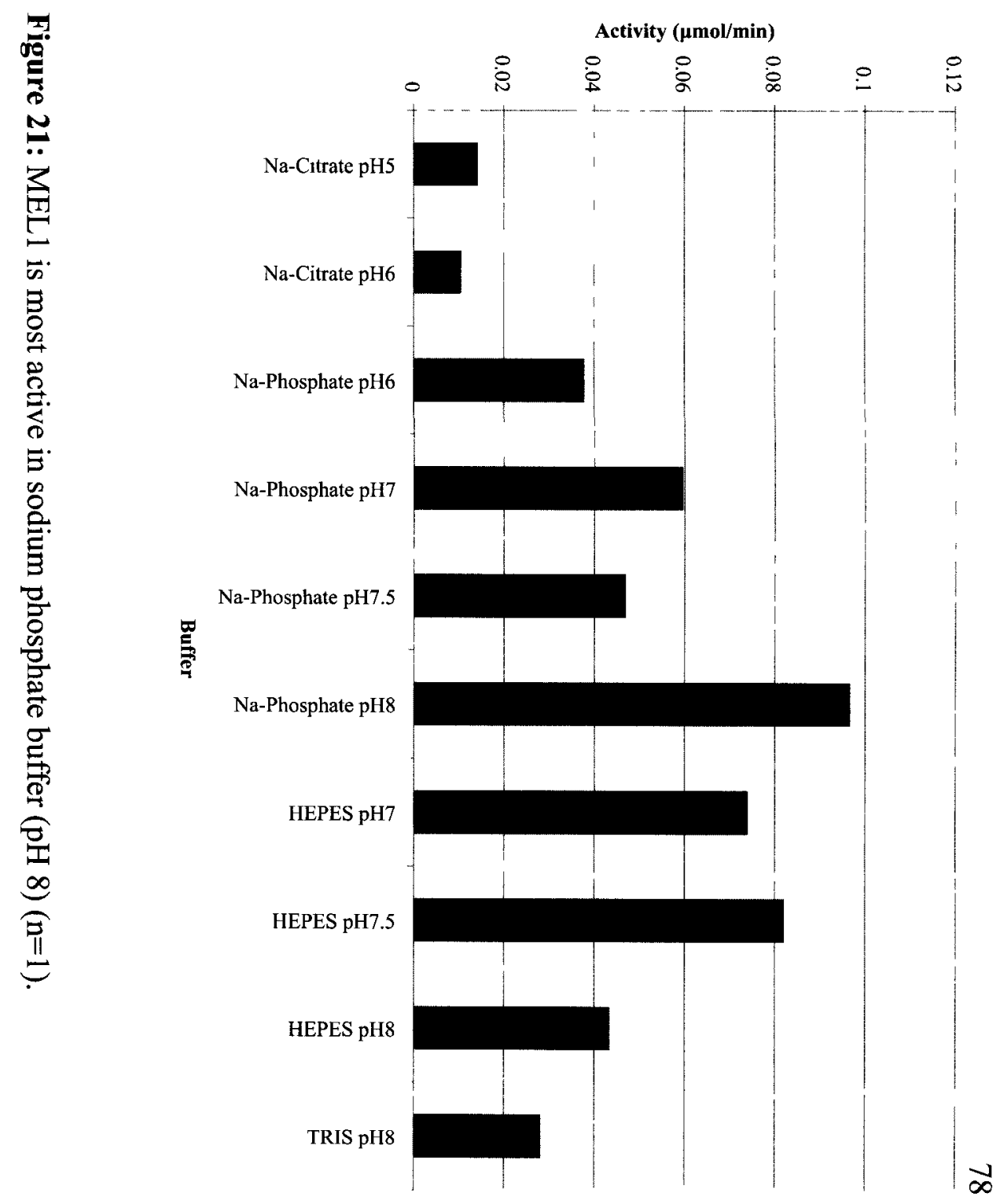
A)

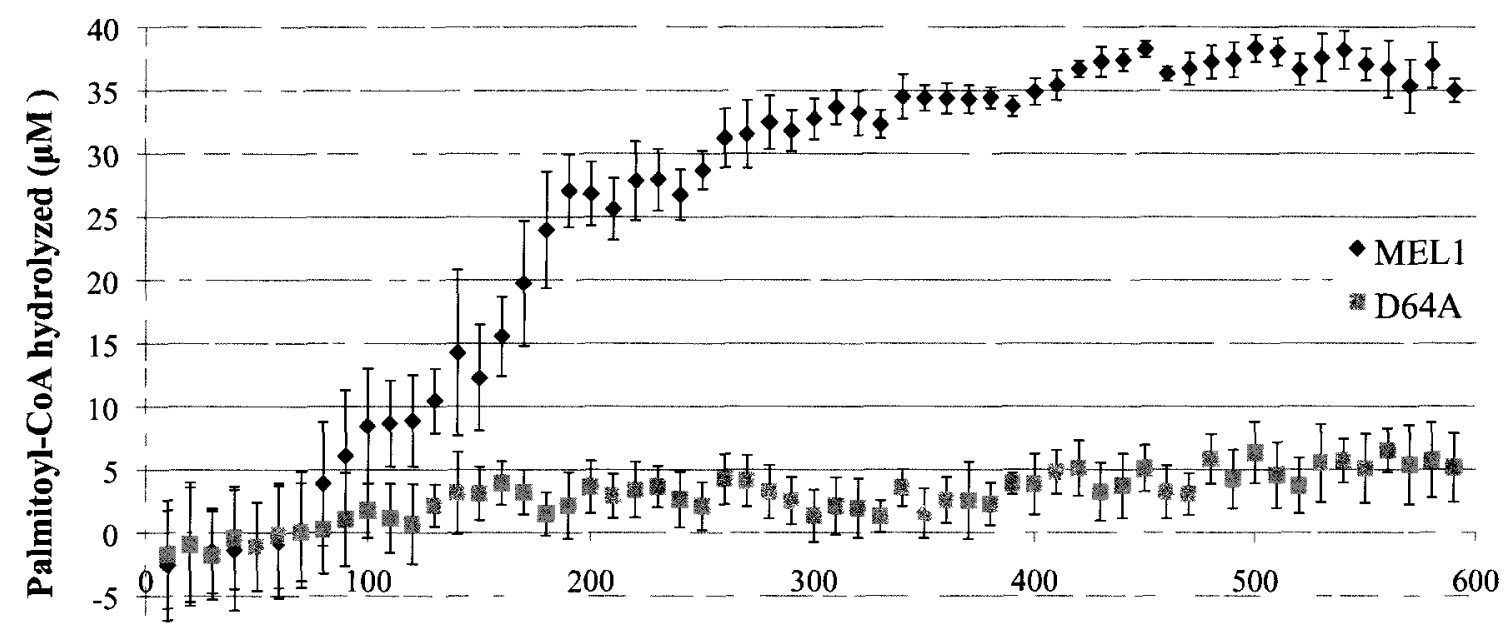
$-10$

Time (min)

B)

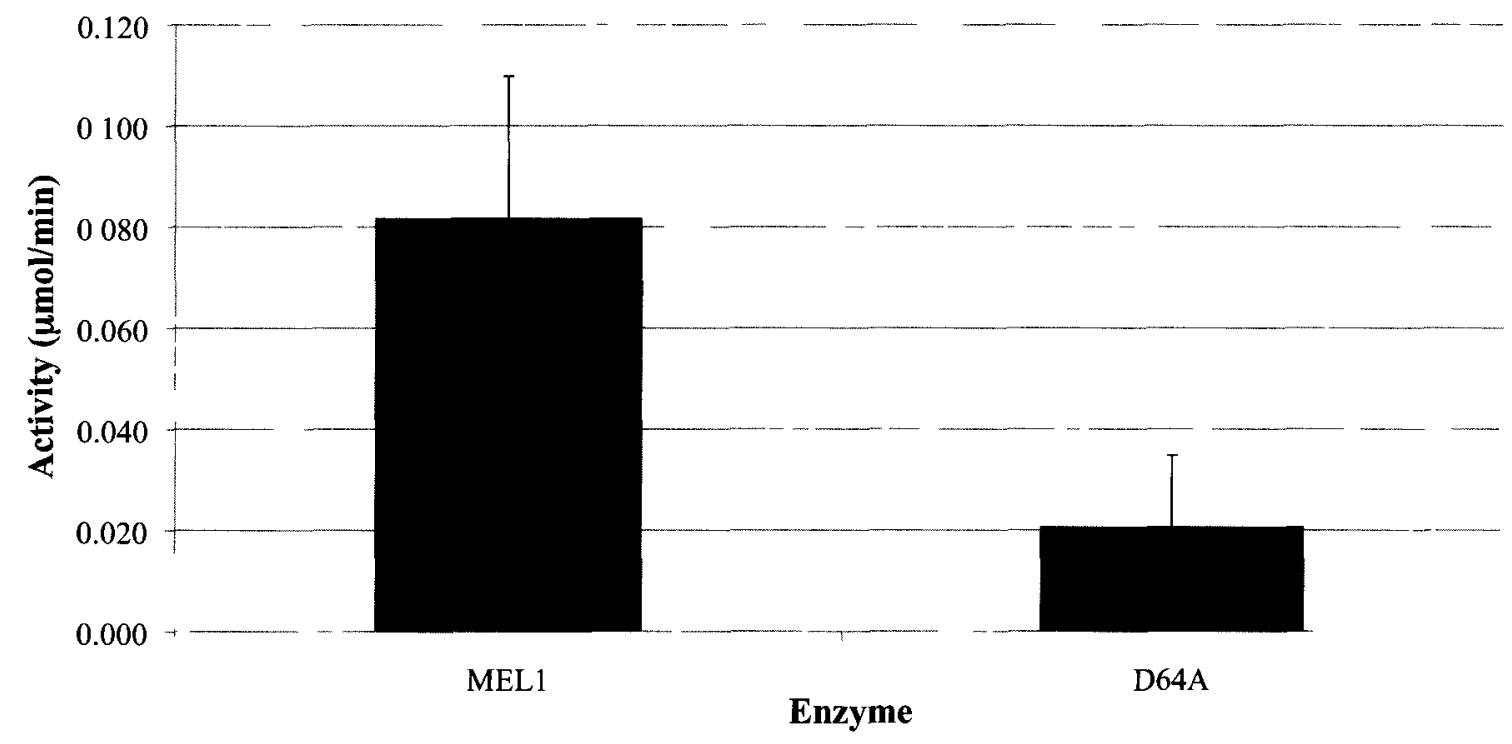

Figure 22: MEL1 is an active thioesterase using palmitoyl-CoA as a substrate.

The overall amount of palmitoyl - CoA hydrolyzed by the MEL1 enzyme is significantly higher then the mutant (A). After $150 \mathrm{~min}$, the activity of MEL1 is much higher then the $\operatorname{MEL1}(\mathrm{D} 64 \mathrm{~A})$ mutant (B) $(\mathrm{n}=3)$. 
3.6 The expression of MEL proteins in K27 E. coli cells leads to the detection of novel compounds in the media.

The MEL proteins were also expressed in K27(DE3) E. coli cells to test for the accumulation of fatty acids in the media. The K27 strain contains a mutation in the FadD gene, which prevents cells from importing fatty acids from the media and thus allows for the accumulation of lipids in the media for analysis by GC-FID (Overath et al., 1969). The K27 cells were made lysogenic for $\mathrm{T} 7$ polymerase to allow inducible expression of proteins by IPTG (Lü et al., 2009). A truncated version of FATB, a known thioesterase, lacking the plastid transit sequence was also expressed in these cells as a positive control for the accumulation of fatty acids (Mayer and Shanklin, 2007). The expression of MEL1, MEL2, MEL3, and MEL1(D64A) in K27(DE3) cells led to significantly higher levels of protein accumulation than observed in BL21(DE3)pLysS cells, such that induced proteins were now easily seen on a coomassie-stained gel (Figure 19A; Figure 23A). Following the expression of the MEL enzymes, the spent media was analyzed by GC-FID for the presence of fatty acids using palmitic acid (C16:0) and stearic acid (C18:0) as retention time standards. Expression of MEL1, MEL2, and MEL3 caused increased amounts of C18:1 fatty acid in the media. MEL1 expression also led to the production of $\mathrm{C} 16: 1$ fatty acid in the media (Figure 24). C16:1 and C18:1 are the primary compounds that accumulate when FATB is expressed in K27(DE3) cells, providing a reference for the retention times of these compounds (Mayer and Shanklin, 2007). In addition to the accumulation of C16:1 and C18:1, the expression of MEL1, MEL2, and MEL3 lead to the accumulation of several other unidentified compounds not observed in either the empty vector or MEL1(D64A) mutant (Figure 24). The activity of 
MEL1 to similar substrates as FATB confirms that the MEL protein family has acyl-ACP thioesterase activity.

The MKS2 protein from $S$. habrochaites, which is $73 \%$ similar to MEL1 at the amino acid level, has recently been characterized and found to have thioesterase activity towards $\beta$-ketomyristoyl-ACP in the tomato methylketone biosynthesis pathway in glandular trichomes (Ben-Israel et al., 2009; Yu et al., 2010). Following expression of MKS2 in BL21(DE3) E. coli cells, several methylketones, including 2-tridecanone (C13:0), were detected in the media following heat and acid treatment (Yu et al., 2010). Due to the similarity between MKS2 and the MEL family of enzymes (Appendix 5), the MEL proteins were initially expressed in BL21(DE3) cells to test for the accumulation of methylketones via a $\beta$-ketoacid intermediate. However, upon discovering that the MEL proteins accumulated to much higher levels in K27(DE3) cells than in BL21(DE3) cells, the K27(DE3) cell line was used for this analysis (Figure 19A; Figure 23A). The expression of MEL proteins in K27(DE3) E. coli cells led to the accumulation of novel compounds in the media. MEL3 expression led to the accumulation of 2-tridecanone (C13:0) and 2-tridecenone (C13:1), with low levels of 2-undecanone (C11:0) and 2petadecenone (C15:1) (Figure 25). The retention time of 2-tridecanone was verified with a commercial standard. However, the retention times associated with 2-tridecenone, 2undecanone, and 2-pentadecenone were estimated based on the data presented by Yu et al. (2010). MEL2 expression led to the accumulation of low levels of the abovementioned compounds and a large amount of an unknown compound with a retention time of 1.82 minutes (Figure 25). While the expression of MEL1 led to the accumulation of 2-undecanone, it did not appear to cause the accumulation of any of the other 
identified methylketones (Figure 25). None of the methylketone compounds analyzed were present in either the vector alone control, MEL(D64A), or FATB-expressing cells, indicating that the accumulation is due to the activities of the MEL proteins (Figure 25). In addition to the presence of known methylketones, the expression of MEL1 and MEL2 lead to the accumulation of several other unidentified compounds in the spent media that were not found in the controls (Figure 25). The presence of methylketones in the media of $\mathrm{K} 27(\mathrm{DE} 3)$ cells expressing the MEL proteins indicates that the MELs are able to hydrolyze the thioester bond in $\beta$-ketoacyl-ACP compounds to form $\beta$-ketoacids, which are then chemically converted to methylketones. 
A)

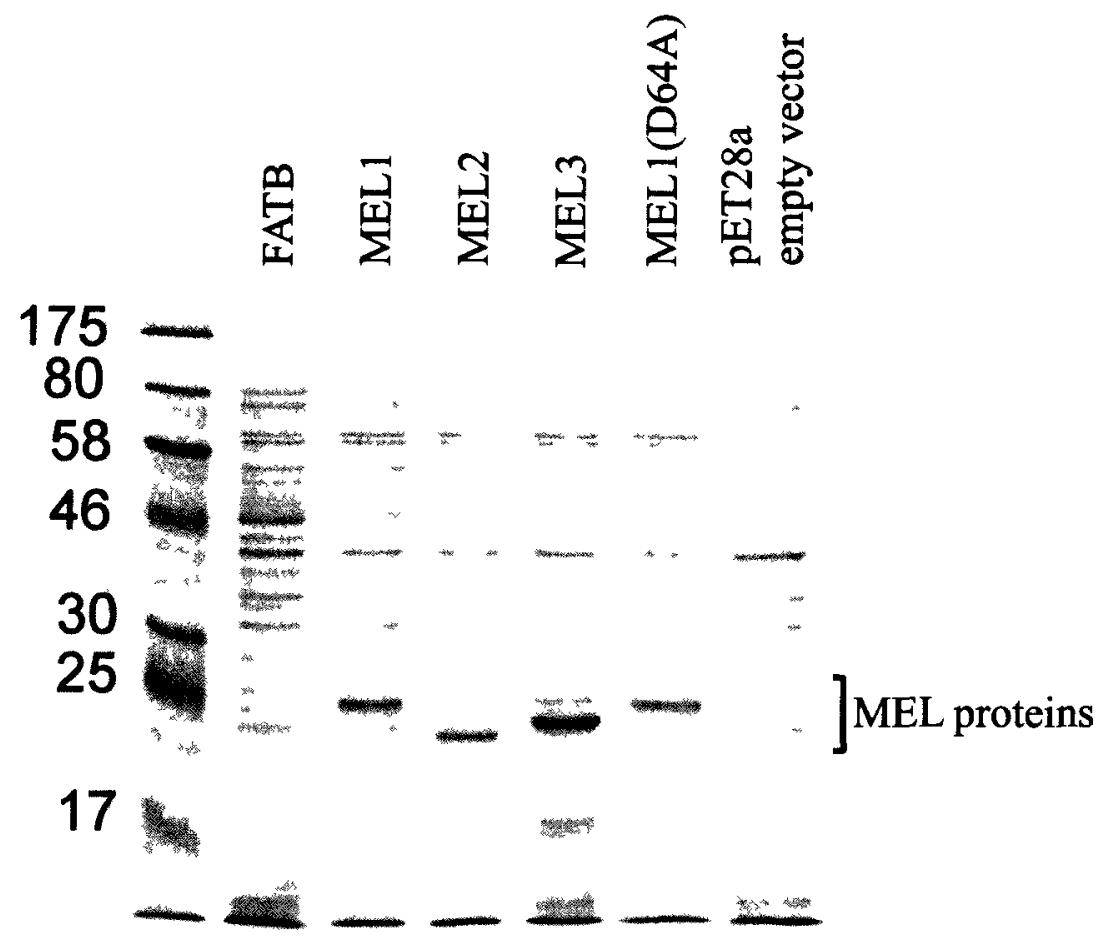

B)

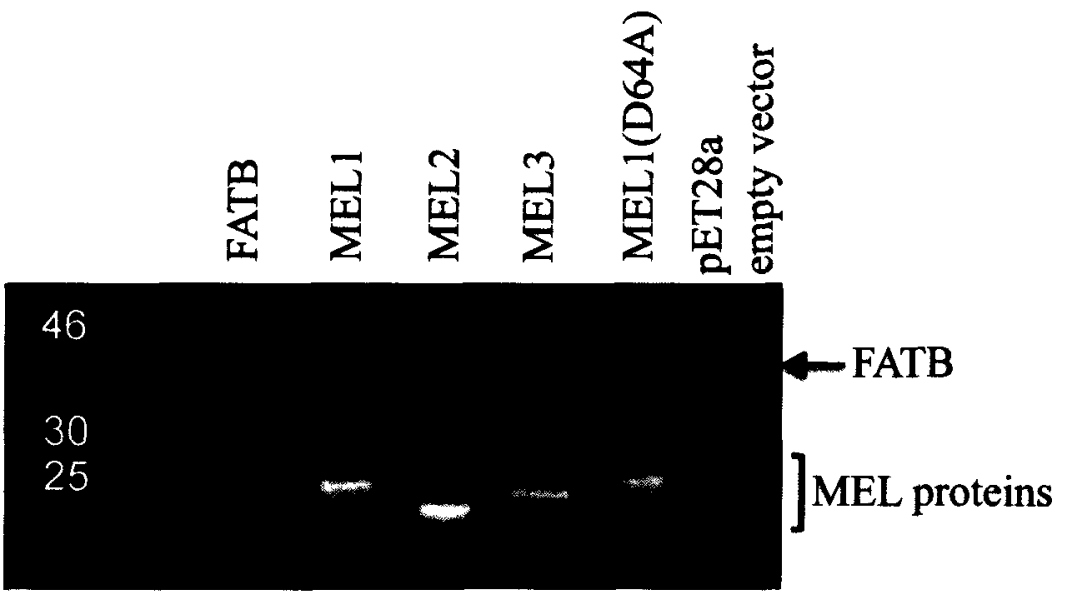

Figure 23: Expression of MEL1, MEL2, MEL3, MEL1(D64A), and FATB in the K27(DE3) E. coli cell line.

Crude protein fractions from MEL1, MEL2, MEL3, MEL1 (D64A) and the empty pET28a vector were run on a $12 \%$ SDS-PAGE gel and stained with Coomassie Blue (A). Western blot analysis was carried out on the protein fractions using an antibody against the T7 epitope (B). 
Figure 24: Expression of MEL proteins in the K27 strain of $E$. coli leads to the accumulation of free fatty acids in the media.

The indicated C16:0 and C18:0 fatty acid retention times of 10.09 and 14.68 minutes, respectively, were confirmed by the retention time of commercial standards. The indicated C16:1 and C18:1 fatty acid peaks were predicted based on data presented by Mayer and Shanklin (2007). Peaks notated with an asterisk are novel peaks not seen in either the empty vector or mutant enzyme, which have yet to be identified. The traces presented are representative of 3 biological replicates. 


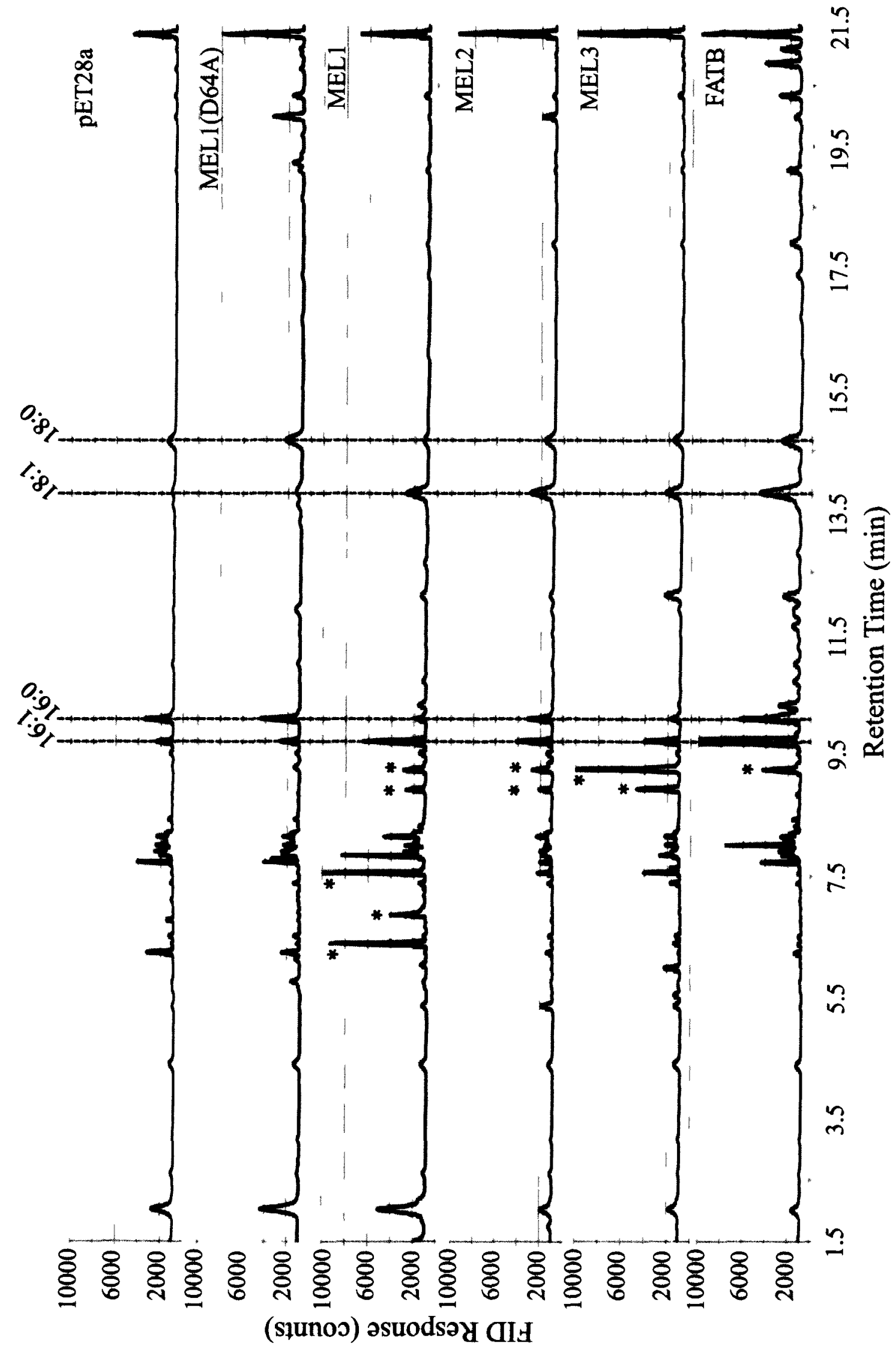


Figure 25: The expression of MEL proteins in the K27 strain of $E$. coli leads to the accumulation of novel $\beta$-ketoacids in the media.

$\beta$-ketoacids are converted to methylketones chemically using $2 \mathrm{M}$ sulfuric acid for quantification as $\beta$-ketoacids are naturally unstable compounds. 2-tridecanone (C13:0) with a retention time of 13.72 minutes was confirmed by the retention time of the commercial standard. The compounds 2-undecanone (C11:0), 2-tridecenone (C13:1), and 2-pentadecenone (C15:1) were predicted based on the data presented in Yu et al. (2010). Peaks notated with an asterisk are novel peaks not seen in either the empty vector or mutant enzyme that have yet to be identified. The traces presented are representative of 3 biological replicates. 


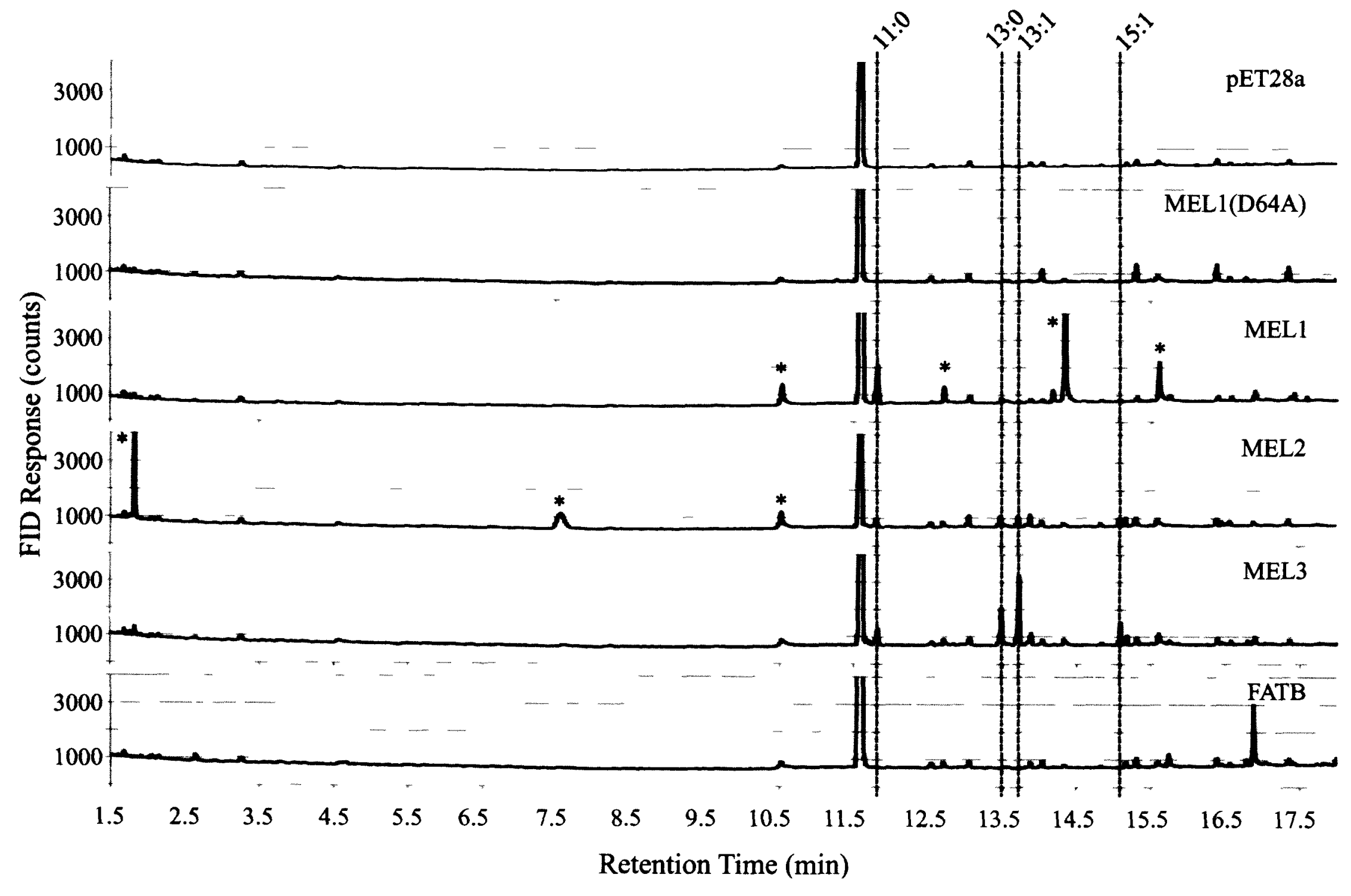




\section{Chapter 4: Discussion}

In this study, I have identified a four member gene family encoding thioesterases whose gene expression patterns are consistent with roles in extracellular lipid biosynthesis. The gene expression patterns of $M E L 1, M E L 2$, and $M E L 4$ were found to be associated with cells that produce cuticle, suberin, and sporopollenin, respectively. This division of roles is also found amongst gene family members encoding other enzyme types involved in generating these surface lipid barriers. The alcohol-forming fatty acylCoA reductase (FAR) family that produces various chain-length primary alcohols in each of the three barriers also shows this division of function (Aarts et al., 1997; Rowland et al., 2007; Domergue et al., 2010). The strong expression of MEL1 in the stem epidermis and flowers is reminiscent of $F A R 3 / C E R 4$, which is involved in synthesizing $\mathrm{C} 24: 0$ to C30:0 primary alcohols found in cuticular wax (Rowland et al., 2006). MEL2, with root endodermal expression, has an expression pattern that is more comparable to FARl, FAR4, and FAR5 that generate $\mathrm{C} 18: 0$ to $\mathrm{C} 22: 0$ primary alcohols associated with suberin (Domergue et al., 2010). Lastly, the expression of MEL4 exclusively in the tapetal cells of the anther is similar to that of FAR2/MS2, which is thought to produce primary fatty alcohols specific for sporopollenin (Aarts et al., 1997). MEL3 was more widely expressed and therefore may have a more general role in lipid metabolism, rather than specific to extracellular lipids.

In cuticle biosynthesis, there are several roles for which thioesterases may be needed. These are: (1) production of free fatty acids (FFA) from C16 and C18 acylACPs in the plastid to allow for export to cytosol, (2) production of free very-long-chain fatty acids following elongation at the ER, (3) production of FFA intermediates leading to 
the synthesis of methylketones and then perhaps to alkanes, (4) production of FFA as a substrate for cytochrome P450 enzymes in the cutin biosynthetic pathway, and (5) production of balanced levels of FFA with the acyl-CoA pool for the control of gene expression of lipid biosynthetic genes as FFAs and acyl-CoAs have been shown to directly influence transcription factor activity in animals and bacteria. Apart from role (1), no thioesterase has yet been identified in Arabidopsis fulfilling any of the other postulated roles. The family of Arabidopsis thioesterases described here may fulfill one or more of these roles as discussed below.

\subsection{Thioesterases in the plastid}

The acyl-ACP thioesterases FATA and FATB in the plastid produce free fatty acids so that the acids can be exported through the plastid envelope and to the cytosol (Salas and Ohlrogge, 2002). Unlike thioesterases found in the ER, plastid localized thioesterases utilize substrates activated with ACP. FATB preferentially hydrolyzes C16:0 fatty acyl-ACP and FATA preferentially hydrolyzes C18:1 fatty acyl-ACP. Loss of the FATB gene in Arabidopsis leads to a 50\% reduction in the overall wax load (Bonaventure et al., 2003). While approximately $50 \%$ of the cuticular wax comes from C16:0 precursors derived from FATB activity, the other 50\% must derive from the activity of some other thioesterase (Bonaventure et al., 2003). Presumably, a portion of the other 50\% comes from $\mathrm{C} 16: 0$ and $\mathrm{C} 18: 0$ fatty acids released via FATA activity, but given that FATA has a preference for C18:1 substrates it is possible that other plastid localized thioesterases are contributing to this $50 \%$ of saturated fatty acids emerging from the plastid (Liu and Post-Beittenmiller, 1995; Salas and Ohlrogge, 2002). Strong 
candidates for these thioesterases may be the MEL proteins. The slow rate of hydrolysis observed with MEL1 toward palmitoyl-CoA may be because MEL1 prefers acyl-ACP substrates. Our evidence in $E$. coli shows that the MEL enzymes are able to utilize longchain acyl-ACP substrates, which supports the hypothesis that MEL enzymes utilize activated substrates in the plastid. Salas and Ohrogge (2002) have shown that the activity of FATB toward acyl-ACP substrates prepared from E. coli is significantly less than its activity toward acyl-ACP substrates prepared from spinach. Therefore, to verify the activity of MEL1 for long-chain acyl-ACPs, long-chain acyl-ACPs should be synthesized from spinach extracts to observe if MEL1 has preferred activity for ACP activated substrates over CoA as presented here.

The MEL family shows high sequence similarity to a tomato enzyme MKS2, which has been shown to catalyze the thioesterase reaction forming free $\beta$-ketoacids ( $\mathrm{Yu}$ et al., 2010). MKS2 localizes to the plastid and therefore methylketones found in the trichomes of $S$. habrochaites are generated from $\beta$-ketoacyl-ACPs, which are intermediates in the fatty acid synthesis pathway (Yu et al., 2010). Following the expression of the MEL enzymes in E. coli, both methylketones and free fatty acids accumulated in the media (Figure 24; Figure 25). The expression of FATB in these cells, however, only led to the accumulation of fatty acids and not methylketones. This indicates that MEL enzymes may have some dual functionality, cleaving ACP from both fully reduced fatty acyl-ACP and $\beta$-ketoacyl-ACP moieties. The ability of single Hotdog fold thioesterases to accommodate multiple substrates in vitro has previously been seen with hTHEM2, a thioesterase found in human mitochondria (Cheng, et al., 2006). The first reported activity of this enzyme identified a preference for aromatic substrates, such 
as 3,4-dihydroxyphenylacetyl-CoA (Cheng, et al., 2006). Subsequent studies however identified an affinity for medium and long-chain fatty acyl-CoA substrates (Wei et al., 2009). MEL enzymes may function primarily in the release of free fatty acids from the plastid, but when presented with $\beta$-ketoacyl-ACPs, free $\beta$-ketoacids can be produced that subsequently form methylketones. However, medium or long-chain methylketones have not been reported amongst the surface lipids of Arabidopsis. In tomato glandular trichomes, MKS2 may be specific for $\beta$-ketoacyl-ACPs and natural selection has lead to the high expression of this gene in trichomes for the accumulation of these methylketones. To test this hypothesis, the kinetics of paltmitoyl-ACP and various $\beta$ ketoacyl-ACP substrates can be compared using MKS2 and the various MEL enzymes. Testing the MKS2 spent media for the presence of free fatty acids would ascertain whether the duel substrate specificity seen with MELs is also present in MKS2. The preparation of the media and GC conditions to detect $\beta$-ketoacids and fatty acids are quite different and therefore fatty acids cannot be detected in samples prepared for methylketone analysis and vice versa.

4.2 Thioesterases in the production of very-long-chain free fatty acids

Very-long-chain free fatty acids are components of the cuticular waxes and account for 3.2\% of total amounts in Arabidopsis stems (Jenks et al., 1995; Pollard et al., 2008). The FAE complex utilizes acyl-chains activated with CoA and therefore a verylong-chain thioesterase would likely be needed to release the free fatty acid for subsequent secretion to the cuticle. In vitro, MEL1 is able to hydrolyze palmitoyl-CoA, but the rate of hydrolysis observed was quite slow (Figure 22). However, MEL1 may be 
more active toward very-long-chain fatty acyl-CoAs instead of long-chain acyl-CoAs. The expression of MEL1, MEL2, and MEL3 in E. coli cells led to the accumulation of a variety of fatty acids in the media including C18:1 and C16:1 (Figure 24). This activity observed in E. coli would suggest that the MELs can utilize monounsaturated long-chain fatty acyl substrates, but these may not be biologically relevant. E. coli do not produce very-long-chain substrates and therefore the activity observed by MEL1, MEL2, and MEL3 could be limited by substrate availability. This has previously been seen with FAR enzymes when expressed in E. coli (Doan et al, 2009). By examining the cuticular waxes of the cer $4 / f a r 3$ mutant, it has been determined that the in planta substrate specificity of CER4/FAR3 is C24:0 to C30:0 acyl-CoAs (Rowland et al., 2006). However, CER4 expression in E. coli caused the accumulation of C18:1 and C16:0 primary alcohols (Doan et al., 2009). Conversely, expression of CER4/FAR3 in yeast yielded C24:0 and C26:0 primary alcohols, which is consistent with the mutant phenotype (Rowland et al., 2006). To further assess the substrate specificities of the MEL proteins, they could be expressed in yeast and the spent media examined for the presence of very-long-chain fatty acids as yeast possess up to $\mathrm{C} 26: 0$ very-long-chain fatty acyl-CoAs. In vitro assays with very-long-chain acyl-CoAs are difficult, if not impossible, due to the hydrophobicity of very-long-chain fatty acids and therefore yeast heterologous expression may be the best technique with which to test the substrate specificities of the various MELs. 
4.3 Thioesterases in the biosynthesis of $\beta$-ketoacids and alkanes

The current proposed model of alkane synthesis involves a two-enzyme system in which the first is an aldehyde-forming FAR and the second is an aldehyde decarbonylase (Figure 26A) (Cheesborough and Kolattukudy, 1984). Evidence for this came from studies in Pisum sativum where partial enzyme purification indicated an aldehyde decarbonylase activity releasing carbon monoxide from the aldehyde intermediate (Cheesborough and Kolattukudy, 1984; Schneider-Belhaddad and Kolattukudy, 2000). There are reports of an aldehyde-forming FAR protein in both $P$. sativum and Botryococcus braunii, but neither corresponding gene has been identified (Wang and Kolattukudy, 1995; Vioque and Kolattukudy, 1997). Compelling evidence of a twoenzyme system utilizing an aldehyde intermediate has been reported in cyanobacteria (Schirmer et al., 2010). However, this mechanism of alkane and aldehyde synthesis remains to be demonstrated in planta (Samuels et al., 2008).

An alternative pathway for alkane synthesis may involve a thioesterase activity. The $\beta$-ketoacyl synthase enzyme in the FAE complex generates $\beta$-ketoacyl-CoAs from fatty acyl-CoAs. In addition to being an intermediate in fatty acid elongation, the $\beta$ ketoacyl-CoAs can also possibly serve as precursors to methylketones in other plant species. In tomato, medium-chain $\beta$-ketoacyl-ACPs are converted to a $\beta$-ketoacid by a thioesterase and the $\beta$-ketoacid is decarboxylated to a methylketone (Fridman et al., 2005; Ben-Israel et al., 2009; Yu et al., 2010). As stated previously, the MEL family shows high similarity to MKS2, which has been shown to catalyze the thioesterase reaction forming free $\beta$-ketoacids (Yu et al., 2010). Using $\beta$-ketoacyl-CoAs as a substrate, MEL1 could catalyze the conversion of $\beta$-ketoacyl-CoAs to $\beta$-ketoacids. It is 
then possible that either an unknown decarboxylase reduces the $\beta$-ketoacid to a methylketone or that this decarboxylation occurs spontaneously (Fridman et al., 2005). An oxidoreductase, such as CER3 and CER1, may then be able to successively reduce the methylketone to form alkanes (Figure 26B). In cer1 mutants, alkanes, secondary alcohols, and ketones are all decreased while aldehydes are increased (Jenks et al., 1995). This could support the proposed alternative pathway, if the loss of the oxidoreductase causes an accumulation of methylketones, which could then inhibit the thioesterase activity. The decreased flux through this branch of the wax biosynthetic pathway could allow for more $\beta$-ketoacyl-CoAs to continue in FAE to form more fatty acyl-CoAs, where they could then be available to a putative aldehyde-forming FAR. In cer3 mutants however, it appears aldehydes, alkanes, secondary alcohols, and ketones are all decreased which doesn't fit perfectly with either model for alkane biosynthesis, unless CER3 itself is involved in aldehyde synthesis (Jenks et al., 1995). Expression of MEL1, MEL2, and MEL3 in E. coli leads to the accumulation of C11 to C15 methylketones in the spent media, in spite of the fact that Arabidopsis does not have detectable levels of medium chain methylketones (Yu et al., 2010). E. coli do not synthesize alkanes and therefore the lack of an oxidoreductase that could convert methylketones into alkanes, would allow for the accumulation of $\beta$-ketoacids that are then chemically converted to methylketones. If methylketones are an intermediate in alkane synthesis, then some plants may have evolved to have increased expression of this methylketone-forming thioesterase in specialized cells (Yu et al., 2010; Perera et al., 2010). The accumulation of methylketones may overwhelm the oxidoreductase, if the oxidoreductase is expressed at all, and therefore an accumulation of methylketones is found in these cells, such as in the 
trichomes of $S$. habrochaites or in the silk cuticular wax of maize (Fridman et al., 2005; Perera et al., 2010).

A thioesterase in alkane synthesis would need to have activity for very-long-chain acyl-CoAs, but expression in E. coli indicates that MEL1, MEL2, and MEL3 utilize medium to long-chain substrates (Figure 25). However, as described above, expression in $E$. coli gives an indication of the activity that the MEL proteins can carry out, but the substrate specificity may not be relevant as very-long-chain acyl substrates are not produced in E. coli. Expressing the MEL proteins in yeast may determine if MEL enzymes can utilize both long-chain and very-long-chain acyl substrates. It has been reported that suberin has associated waxes, including alkanes, and therefore MEL2 could be functioning in the production of suberin waxes while MEL4 is potentially generating alkanes for the pollen exine (Li et al., 2007b). Alkanes specifically within the pollen exine have not been reported, but this is difficult to measure experimentally due to low tissue availability. Alkanes, however, have been reported associated with tapetal cells and are thought to coat the pollen surface in the final stages of pollen grain formation (Hsieh and Huang, 2007). The mutants that affect alkane levels, cer3 and cerl, show male sterility, indicating a critical role for alkanes in pollen viability.

It is possible that the MEL enzymes carry out the same function as MKS2 in tomato and are indeed primarily responsible for the synthesis of methylketones. Methylketones in tomato can be detected due in part to the high levels at which they accumulate in these glandular trichomes (Yu et al., 2010; Ben-Israel et al., 2009; Fridman et al., 2005). It is possible that long-chain and very-long-chain methylketones are present in Arabidopsis but at levels that are not yet detectable. 
A) Current alkane pathway

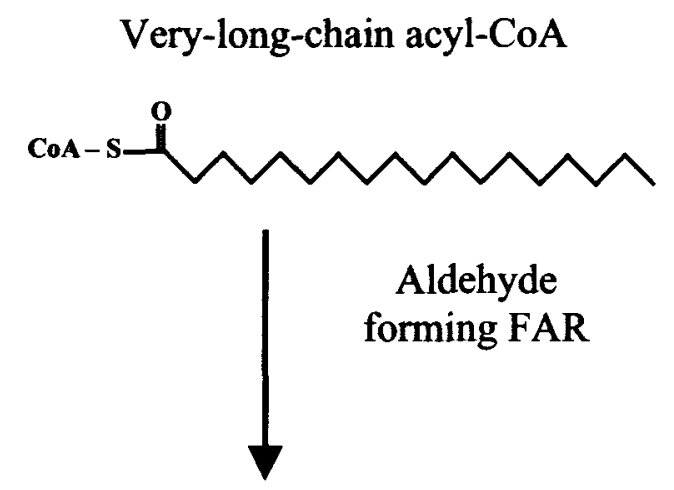

Aldehyde

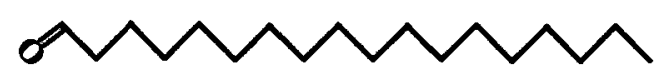

CO Decarbonylase

Alkane

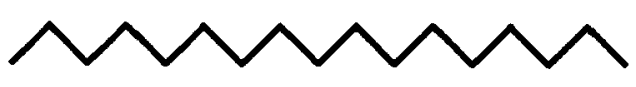

B) Alternative alkane pathway

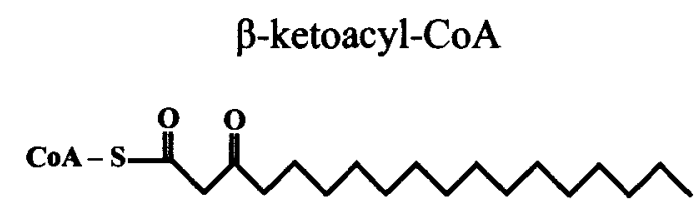

Thioesterase

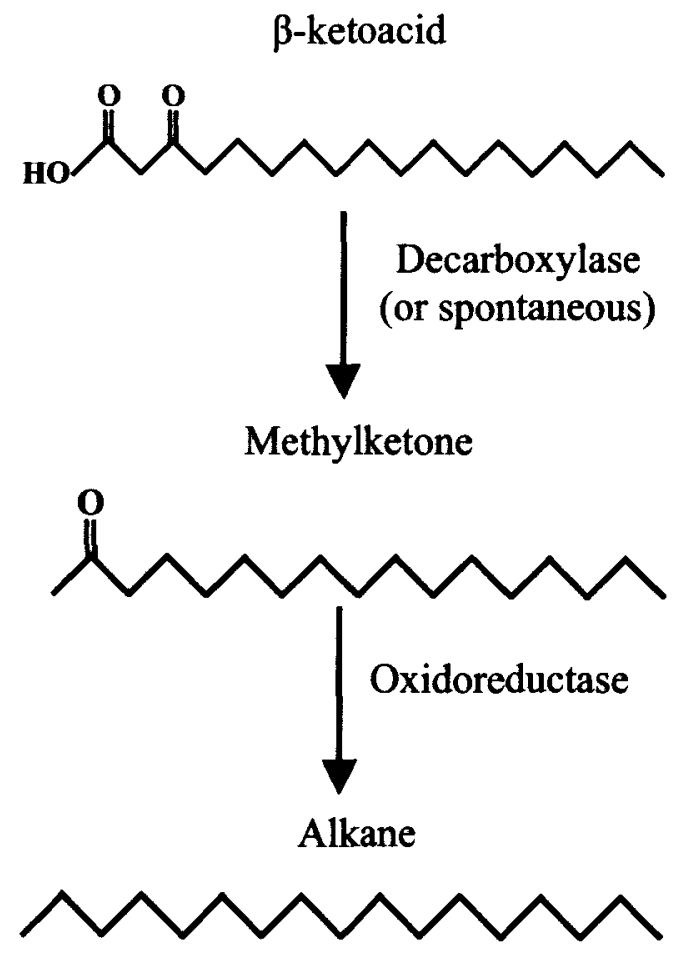

Figure 26: Two models for alkane biosynthesis in plants.

The current hypothesis for alkane formation involves an aldehyde-forming fatty acylCoA reductase and a decarbonylase (A). The alternative alkane biosynthetic pathway involves an acyl-CoA thioesterase and a methylketone intermediate (B). 
4.4 Thioesterases in cutin biosynthesis

Many of the enzymes associated with cutin biosynthesis have been identified, but the sequence of reactions in cutin monomer synthesis is currently unknown (see section 1.2.1). In one model, a long-chain thioesterase is needed to generate a free fatty acid from the acyl-CoA pool, which is then subsequently oxidized by a cytochrome P450 to a hydroxy-fatty acid and reactivated to a hydroxy fatty acyl-CoA before being transferred to glycerol (Figure 4A). The known P450 hydroxylases ATT1 and LCR in Arabidopsis that are associated with cuticle biosynthesis have demonstrated activity toward free fatty acids in vitro and ATT1 does not appear to have any activity toward acyl-CoAs (Benveniste et al., 2006; Wellesen et al., 2001). This indicates that the activated acylCoA substrates in the ER are likely converted to a free fatty acid prior to hydroxylation. These free hydroxy-fatty acids would need to be reactivated by a LACS, such as LACS1 or LACS2, for the GPAT to form the mono and diacylglycerolipids (Lü et al., 2009). This sequence of reactions is supported by data indicating that LACS2 has some activity for C16:0 fatty acids, but higher activity for hydroxylated C16 fatty acids (Schnurr et al., 2004). While this sequence of reactions is supported in Arabidopsis, recent work in Petunia hybrida presents an alternative. The fatty acyl-CoA $\omega$-hydroxylase, CYP86A22, identified as important for the synthesis of $\omega$-hydroxy fatty acids in the $P$. hybrida stigma preferentially hydroxylates long-chain fatty acyl-CoAs over free fatty acids in the synthesis of flower cutin. This is unlike the known hydroxylases from Arabidospsis that seem to use free fatty acids as their substrate (Han et al., 2010). However, the authors note that the use of activated CoA substrates is unusual for a cytochrome P450 hydroxylase. If the hydroxylation of fatty-acyl-CoAs is occurring in Arabidopsis, there 
would be no need for a thioesterase as free fatty acids would be activated to a fatty acylCoA, which could then be hydroxylated and transferred to glycerol. Support for this alternative pathway, however, is weak. In vitro, MEL1 has activity for palmitoyl-CoA, and long-chain fatty acids are produced when MEL proteins are expressed in E. coli. This illustrates that the MEL enzymes can use long-chain acyl chains as substrates. Hydroxylated fatty acids are also found in suberin and most likely sporopollenin (Dominguez et al., 1999; Franke et al., 2005). Similar to the cutin biosynthetic pathway, two fatty acid hydroxylases, CYP86A1 and CYP86B1, contribute to the biosynthesis of hydroxy fatty acids in suberin, one of which has been shown to be active toward mid- and long-chain fatty acids (Benveniste et al., 1998; Compagnon et al., 2009). This indicates that the hydroxylated fatty acids found in suberin are likely synthesized through a mechanism analogous to that seen in Arabidopsis cutin biosynthesis and MEL2 is a candidate thioesterase in this pathway. Due to the similarities between cutin and suberin biosynthetic pathways, it is likely that the biosynthesis of sporopollenin is similar and therefore MEL4 may generate free fatty acids needed for generating hydroxylated fatty acids in sporopollenin. Based on this model there could be a role for MEL enzymes in the production of hydroxylated fatty acids found in the three extracellular lipid barriers.

\subsection{Thioesterases in gene regulation}

Fatty acyl-CoAs and free fatty acids have also been implicated in gene regulatory roles in both bacteria and humans. In humans, the human nuclear factor (HNF)-4 $\alpha$ and peroxisome proliferator-activated receptor (PPAR) $\alpha$ are transcription factors regulating lipid metabolism (Desvergne and Wahli, 1999). The HNF-4a transcription factor is 
activated by mid- to long-chain saturated and mono-unsaturated fatty acyl-CoA ligands, but is suppressed by long-chain polyunsaturated fatty acids (Hertz et al., 2005). The thioesterase activity possessed by HNF-4 $\alpha$ may serve as a mechanism for the cell to monitor the state of cellular acyl-CoA pools and prevent over-stimulation of the downstream targets (Hertz et al., 2005). The PARR $\alpha$ enzyme from animal cells also functions in the lipid metabolism pathway and responds to the pools of free fatty acids and fatty-acyl-CoAs in the cell. In contrast to HNF-4 $\alpha$, it appears that PPAR $\alpha$ has decreased activity in the presence of all long-chain acyl-CoA substrates and is thought to be activated by free-fatty acids (Keller et al., 1992; Elholm et al., 2001; Murakami et al., 2001). A similar regulatory mechanism controls fatty acid metabolism in bacteria (Fujita et al., 2007). The FapR protein functions as a repressor for many lipid biosynthetic genes and binds both malonyl-CoA and malonyl-ACP used in fatty acid synthesis (Schujman et al., 2006; Martinez et al., 2010). Malonyl-CoA is used to initiate fatty acid biosynthesis, but for further fatty acid synthesis malonyl-CoA must be converted to malonyl-ACP (Fujita et al., 2007). The binding of the malonyl-CoA or malonyl-ACP ligands to FapR causes a conformational change that relieves the repression of many lipid metabolism genes. The accommodation of ligands needed for two different steps of the lipid biosynthetic pathway may be a mechanism for monitoring the progression of lipid biosynthesis (Schujman, et al., 2006; Martinez et al., 2010). These are two known examples of fatty acyl-CoAs/FFAs functioning as allosteric transcriptional modulators of lipid metabolic genes. It is possible that the MEL enzymes, or another thioesterase, are functioning in conjunction with a LACS to regulate lipid metabolism by maintaining balanced levels of acyl-CoA pools. 


\subsection{Analysis of the $M E L$ gene family}

If MELs were involved in extracellular lipid biosynthesis, it was expected that the cutin or cuticular wax content of MEL1 amiRNA or MEL1-35S lines would be altered. However, this was not the case, as the cutin and wax composition of MELI amiRNA and MEL1-35s lines were indistinguishable from wild-type (Figure 16; Figure 18). In the case of the over-expression line, it is possible that while MEL1 transcript is increased, the protein level is not increased significantly enough to cause observable phenotypic change. The lack of phenotype in MELI amiRNA lines may be due to incomplete silencing of MEL1. In maize, RNAi was used to decrease the expression of an epidermal specific transcription factor and in spite of significant decreases in transcript, no phenotype was observed (Javelle et al., 2010). This could also be the case in the MEL1 amiRNA lines, where even a small amount of protein may be able to function well enough to prevent any obvious phenotype. MEL3 and MEL1 may also function redundantly and therefore the constitutive expression of $M E L 3$ may mask phenotypes that could otherwise be observed by knocking down MEL1. Examining a plant in which both of these genes are knocked down would test this hypothesis. If these two enzymes are truly redundant, then it would be expected that the compounds produced by their expression in E. coli would be very similar if not identical. However, when MEL1 and MEL3 were expressed in $E$. coli only a few shared compounds, such as undecanone and C18:1 fatty acid, accumulated (Figure 24; Figure 25). The overall profile of compounds produced by these enzymes is quite different and therefore it is unlikely that the two have enough redundancy to mask a phenotype. 
It is possible that other thioesterases functioning in the same pathway are partially redundant, which would also mask any phenotype seen in the MEL1 amiRNA lines. If MEL enzymes are functioning redundantly with FATB in the export of fatty acids from the plastid, it is possible that FATB activity masks the effects of reduced $M E L I$ transcript as FATB is known to be responsible for a large portion of epicuticular wax precursors (Bonaventure et al., 2003). While there is a role for a thioesterase in many aspects of extracellular lipid biosynthesis, as outlined above, the data presented by this study does not resolve these alternate roles (see Chapter 5 for future directions). Preliminary data from experiments carried out after the completion of this thesis has shown that MEL1 is likely localized to the plastid (Alia Busuttil and Owen Rowland, personal communication). Plastid localization has also been seen in the related MKS2 enzyme from tomato (Yu et al., 2010). This does not support MELs function in the roles of modifying acyl-chains for wax, cutin, or suberin biosynthesis following the export of long-chain fatty acids from the plastid, as all evidence thus far indicates that modification to these monomers takes place in the ER (Samuels et al., 2008). However, it does indicate that MELs may be playing a role parallel to FATB in fatty acid synthesis, or in producing mid- to -long-chain methylketones, but these have not yet been identified in the Arabidopsis cuticle. MEL enzymes are likely still functioning in extracellular lipid biosynthesis as their gene expression patterns are so tightly correlated with other genes involved in surface lipid barriers. 


\section{Chapter 5: Conclusion and Future Directions}

This work has demonstrated that the MEL family of enzymes possess thioesterase activity in vitro and when heterologously expressed in E. coli. MEL1 and MEL2 coregulate with cuticle and suberin genes, respectively, and $M E L 1, M E L 2$, and $M E L 4$ are expressed in a tissue specific manner that suggests their involvement in extracellular lipid biosynthesis. In $M E L 1$ knockdown lines, there were no observable alterations to the cuticle composition. MELI may, however, still function in cuticle biosynthesis, as these lines are not null mutants. Alternatively, MEL1 could be functioning in the production of compounds currently unidentified in the cuticle matrix, such as methylketones, or redundantly with other lipid biosynthetic enzymes such as FATB. Future experiments are needed to fully elucidate the function of MEL1 and the other MEL family members.

5.1 Future experiments to determine the physiological role of $M E L$ genes

In order to elucidate the in vivo function of the $M E L$ family of genes, several experiments can be carried out. While there is no obvious phenotype when MELI is knocked down, it is possible that knocking down MEL2, MEL3, or MEL4 may produce a more distinct phenotype. Therefore amiRNA lines specific for $M E L 2, M E L 3$, and $M E L 4$ should be generated and screened for altered lipid composition. Specifically, the suberin composition in a MEL2 knockdown line and the lipid associated with sporopollenin in a MEL4 knockdown line should be examined. The decreased function of a thioesterase in the suberin or sporopollenin pathways may provide stronger phenotypes than in the cuticle. There also may be less potential redundancy with other thioesterases, such as MEL3, in these tissues. A hairpin RNA with less specificity for one specific $M E L$ 
transcript may knockdown the entire $M E L$ family and any observed phenotype may give an indication of MEL protein function, even if it is pleiotropic. While losing specificity is a disadvantage to the hairpin RNA, it could be advantageous to knockdown $M E L 3$ in conjunction with the other $M E L s$.

The recent finding that MEL1 may be localized to the plastid leads to the hypothesis that MEL1 is working in parallel with FATB in the export of saturated fatty acids from the plastid. Crossing MEL1 amiRNA-4 with fatb could provide valuable insight. If the fatb phenotype is unaltered by the loss of $M E L 1$, then it is likely that MEL1 is not functioning in terminating fatty acid synthesis. However, if a more dramatic phenotype is observed in the mell fatb double mutant, this would support MEL1 and $F A T B$ being partially redundant. Similarly, over-expressing $M E L 1$ in the fatb mutant background could illustrate the same principle. If MEL1 is functionally redundant with $f a t b$ and is a long-chain thioesterase terminating fatty acid synthesis, then over-expressing $M E L 1$ in the fatb background could rescue or partially rescue the mutant phenotype (Bonaventure et al., 2003). The same principle can be applied to examine if MEL1 is playing a regulatory role. The win1/shn1 mutant affecting an AP2-type transcription factor shows altered cuticle composition, but not completely altered regulation. Therefore, other mechanisms are likely involved in regulating genes involved in extracellular lipid biosynthesis (Kannangara et al., 2007). Crossing MEL1 amiRNA-4 with the winl/shnl mutant may lead to a more severe phenotype if both of these potential aspects of regulation are being affected. In order to determine if $M E L 1$ is carrying out a regulatory role, real time RT-PCR could be used to detect if the transcript levels of various cutin and wax biosynthetic genes are altered by the decreased or increased 
expression of MEL1. The lack of phenotype may be due to the fact that altering MELI expression does not cause large changes to gene expression, but their mis-regulation may still be detectable by sensitive methods.

Cutin analysis by GC-MS should be carried out on the identified MEL1 overexpression line to observe if any alterations to the cutin composition have occurred due to increased MEL1 expression. If $M E L 1$ is influencing cutin biosynthesis, the overexpression line may provide crucial information about the role it may play in cuticle biosynthesis, even if amiRNA lines do not reduce transcript levels enough to see a phenotype. Cutin analysis by GC-MS of MEL1 amiRNA-1 and MEL1 amiRNA-4 should also be repeated with a larger amount of tissue to increase the ability to accurately detect the levels of the various compounds.

To get better insight into MEL3 gene expression patterns, a larger portion of the genomic sequence upstream of the MEL3 start codon should be used to drive expression of a reporter gene (e.g. GUS). The expression patterns observed with promoter GUS for MEL1, MEL2, and MEL4 are consistent between individual transformants and matches endogenous transcriptional patterns measured by RT-PCR and DNA microarrays, but this consistency has not been seen with MEL3 promoter:GUS lines (Schmid et al. 2005, Winter et al., 2007). A larger portion of genomic sequence may possess promoter elements that were not present in the first construct and therefore a consistent gene expression pattern may be observed. This expression will allow us to determine in which cell types that $M E L 3$ is specifically expressed in or whether it is constitutively expressed in all cell-types. This may provide clues as to the physiological role of MEL1 in lipid biosynthesis. 
5.2 Experiments to biochemically examine MEL enzymes

Biochemical assays are also needed to further elucidate the biological function of the MEL proteins. The expression of MEL1, MEL2, and MEL3 in E. coli led to the production of known methylketones and fatty acids (Figure 24; Figure 25). However their expression also lead to the production of several unidentified compounds. These unknown compounds should be identified by GC-MS. As the profile of unknown compounds is unique to each expressed MEL, identifying these compounds could help elucidate the different functions of each of these enzymes, especially in differentiating MEL3 from the other MEL proteins as MEL3 appears to be globally expressed.

It has been shown previously that when enzymes specific for very-long-chain fatty acyl-CoAs are expressed in E. coli, novel compounds are produced that do not necessarily represent the in planta substrate specificity (Doan et al., 2009). This is likely due to the fact that very-long-chain-fatty acyl substrates are not present in E. coli and therefore the substrates available to the enzymes are limited to long-chain-fatty acylACPs. The expression of lipid biosynthetic genes in yeast may be more physiologically relevant than expression in $E$. coli as yeast are capable of elongating fatty acids to very long chain lengths. The expression of MEL1, MEL2, MEL3, and MEL4 in yeast, as opposed to $E$. coli, could give further indications as to the physiological function of these enzymes. Analyzing the spent media from yeast for the presence of fatty acids or methylketones could support or refute the evidence observed in E. coli.

The observation that the MEL proteins accumulate to much higher levels in K27(DE3) cells then in BL21(DE3)pLysS is intriguing. This could be due simply to a 
higher expression of T7 RNA polymerase (unknown at present), but it may in fact be linked to the $f a d D$ mutation in the $\mathrm{K} 27$ cells. The fadD mutation prevents $E$. coli from importing fatty acids from the media, allowing fatty acids to accumulate outside the cell. High levels of free fatty acids within the cell are typically toxic to organisms and by preventing the accumulation of fatty acids in the cell, it is possible the MEL proteins are able to accumulate to higher levels without causing lethality (Neal et al., 1965; Wu et al., 2006). Therefore all four MEL proteins and the mutant MEL1(D64A) should be purified from K27 cells to yield increased amounts of pure protein and then thioesterase activity assays carried out. With purer enzyme, a selection of substrates could be tested to observe if the MEL proteins preferentially hydrolyze one substrate over another. Many of the long-chain acyl-CoA substrates are available commercially; however, to test the ability of the MELs to hydrolyze $\beta$-ketoacyl-CoAs, very-long-chain acyl-CoAs, or acylACPs would require substrate synthesis in the lab. Testing MELs for their activity toward long-chain fatty acyl-CoAs, very-long-chain fatty acyl-CoAs, long-chain $\beta$ ketoacyl-CoAs, very-long-chain $\beta$-ketoacyl-CoAs, long-chain fatty acyl-ACPs, and longchain $\beta$-ketoacyl-ACPs could indicate a potential physiological function. Based on the preliminary data that MEL1 is localized in the plastid, it is likely that the native substrate is an acyl-ACP but this should be confirmed by comparing its kinetics with acyl-CoA substrates. Comparing the affinity of various MELs for various fatty-acyl substrates and $\beta$-ketoacyl substrates can distinguish if methylketone production is a non-biologically relevant byproduct of fatty-acyl thioesterase activity or if the synthesis of $\beta$-ketoacids is the primary function of the MEL proteins. 
The characterization of the $M E L$ gene family will identify the role of a previously uncharacterized family of thioesterases. The characterization of thioesterases in plants has thus far been minimal in spite of the multitude of physiological roles for thioesterases. Three Arabidopsis thioesterases have been characterized to date, FATA, FATB, and Acyl-CoA thioesterase 2, and one from tomato, MKS2 (Salas and Ohlrogge, 2002; Tilton et al., 2004; Ben-Israel et al., 2009; Yu et al., 2010). Due to the general lack of knowledge surrounding plant thioesterases, characterizing this new family of acyl thioesterases greatly contributes to the understanding of lipid metabolism in plants. Elucidating how the MEL family contributes to extracellular lipid biosynthesis will allow for a better understanding of these pathways and help direct future research. 
Appendix 1: Primer list

\begin{tabular}{|c|c|}
\hline Primer Name & Primer Sequence \\
\hline MEL1_BamHI_Fwd & AAAGGATCCATGCTTAAAGCTACCGGCACAG \\
\hline MEL1_EcoRI Rev & AAAGAATTCTCAATATTCGACAACGTGTTGAC \\
\hline MEL2 BamHI Fwd & AAAGGATCCATGTTTCAAGCTACCAGCAC \\
\hline MEL2 EcoRI_Rev & AAAGAATTCTCAATCGACCAACTGTTGAC \\
\hline MEL3_BamHI_Fwd & AAAGGATCCATGTTTCTTCAGGTTACCGGCA \\
\hline MEL3_EcoRI Rev & AAAGAATTCTCAAACGGCGTCGTCTTGG \\
\hline FatB_Trunc_SacI_Fwd & AAGAGCTCTTACCTGACTGGAGCATGCTTCTTGC \\
\hline FatB HindIII Rev & GCAAGCTTGGTAGTAGCAGATATAGTT \\
\hline MEL1 D64A Fwd & TTATGAATTAGCCCAATTTGGT \\
\hline MEL1 D65A Rev & CACCAAATTGGGCTAATTCATAA \\
\hline MEL1 Antisense Fwd & ATGCTTAAAGCTACCGGCACAGT \\
\hline MEL1 Antisense Rev & CATAATACGACTCACTATAGGTCAATATTCGACAACGTG \\
\hline MEL1_sense_Fwd & CATAATACGACTCACTATAGGATGCTTAAAGCTACCGGC \\
\hline MEL1_sense Rev & TCAATATTCGACAACGTGTTGACG \\
\hline MEL1_RT Fwd & ATGCTTAAAGCTACCGGCACAGT \\
\hline MEL1 RT_Rev & AACTGTCCACCTCTCCCGAC \\
\hline MEL2 RT Fwd & ATGTTTCAAGCTACCAGCACG \\
\hline MEL2_RT_Rev & TATAGGTCATAAATTTTGTGATGCCC \\
\hline MEL3 RT Fwd & CGAAAACCCAGAAATGTTTCTTCAGG \\
\hline MEL3 RT Rev & TCGCTTTCACCACGAATTTGT \\
\hline GAPC Fwd & TCAGACTCGAGAAAGCTGCTAC \\
\hline GAPC Rev & GATCAAGTCGACCACACGG \\
\hline MEL1 OE XbaI Fwd & AAATCTAGAATGCTTAAAGCTACCGGCACAG \\
\hline MEL1 OE SacI Rev & AAAGAGCTCTCAATATTCGACAACGTGTTGAC \\
\hline MEL1_Prom_SalI_Fwd & AAAGTCGACATGCATCGTTCACCATATCCAC \\
\hline MEL1_Prom_BamHI_Rev & AAAGGATCCTGTGCCGGTAGCTTTAAGCAT \\
\hline MEL2_Prom_SalI_Fwd & AAAGTCGACCACCTTCTCGTAATACAAAGTTTCTC \\
\hline MEL2_Prom_BamHI_Rev & AAAGGATCCCGTGCTGGTAGCTTGAAACA \\
\hline MEL3 Prom Sall Fwd & AAAGTCGACGAGCAAGATCCCATCTCCCTAAAC \\
\hline MEL3 Prom BamHI Rev & AAAGGATCCAACCTGAAGAAACATTTCTGGGTTTTC \\
\hline MEL4 Prom Sall Fwd & AAAGTCGACCATTTAGAGCGATCCCTTACTCATG \\
\hline MEL4 Prom BamHI Rev & AAAGGATCCTGAAGTCGGAAACACCACAGACATA \\
\hline MEL1 ami-A I & GATTAGAGCGTATGTAAGACGGCTCTCTCTTTTGTATTCC \\
\hline MEL1 ami-A II & GAGCCGTCTTACATACGCTCTAATCAAAGAGAATCAATGA \\
\hline MELl ami-A III & GAGCAGTCTTACATAGGCTCTATTCACAGGTCGTGATATG \\
\hline MEL1 ami-A IV & GAATAGAGCCTATGTAAGACTGCTCTACATATATATTCCT \\
\hline MEL1 ami-B I & GATTGACCGAAATTAGAGCGTACTCTCTCTTTTGTATTCC \\
\hline MEL1_ami-B_II & GAGTACGCTCTAATTTCGGTCAATCAAAGAGAATCAATGA \\
\hline MEL1 ami-B III & GAGTCCGCTCTAATTACGGTCATTCACAGGTCGTGATATG \\
\hline MEL1 ami-B IV & GAATGACCGTAATTAGAGCGGACTCTACATATATATTCCT \\
\hline pRS300 A & CTGCAAGGCGATTAAGTTGGGTAAC \\
\hline
\end{tabular}


pRS300 B

GCGGATAACAATTTCACACAGGAAACAG

Ami adapterA XbaI AAATCTAGACAAACACACGCTCGGACGC

Ami adapterB SacI

AAAGAGCTCCCCCATGGCGATGCCTTAA 


\section{Appendix 2: Vector maps of constructs}

NOS terminator, term.; NOS promoter, Pr; 35S enhancer sequence, E6; Kanamycin resistance, KanR; BASTA resistance, BAR; Hygromycin resistance, HygR.

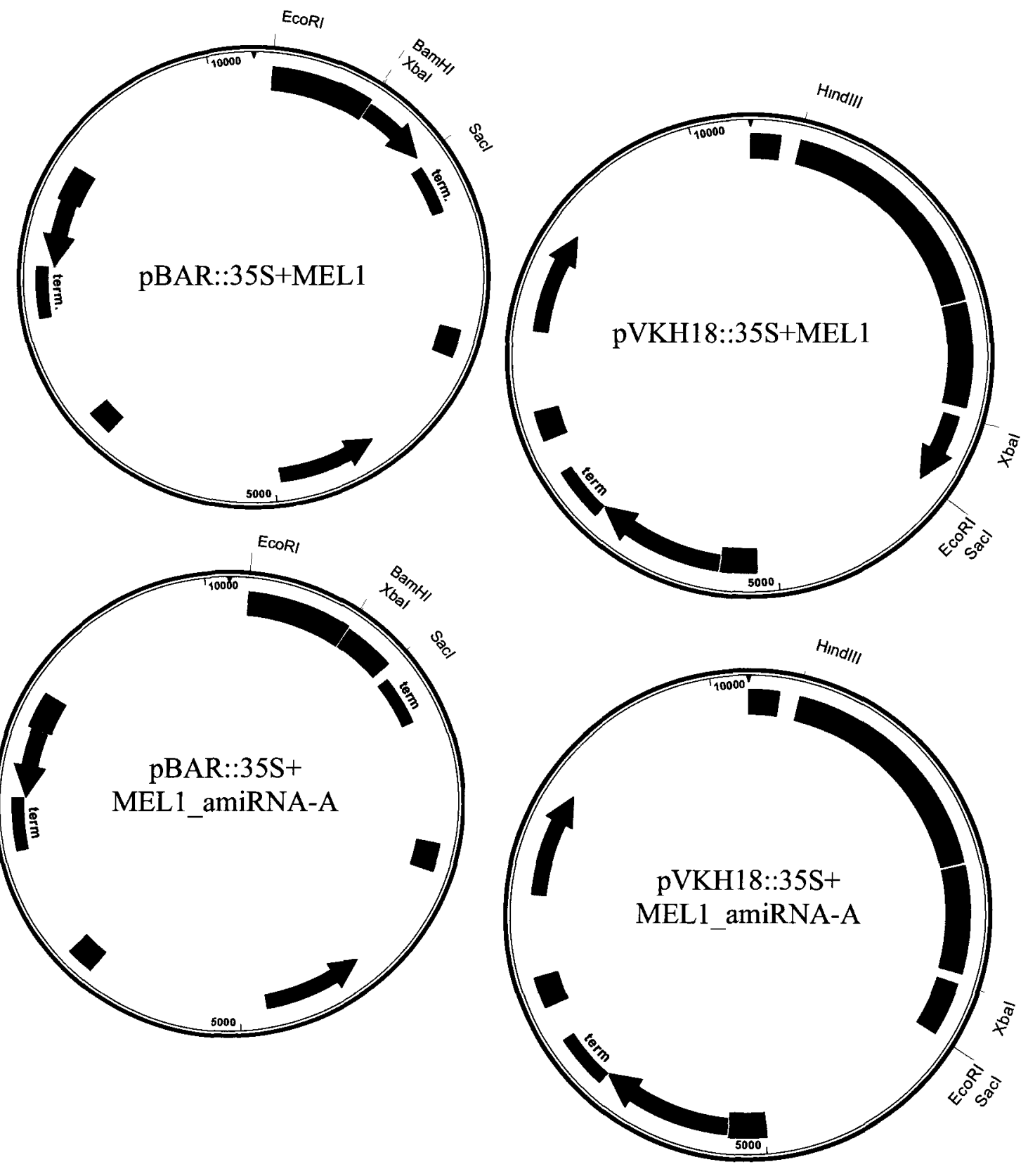




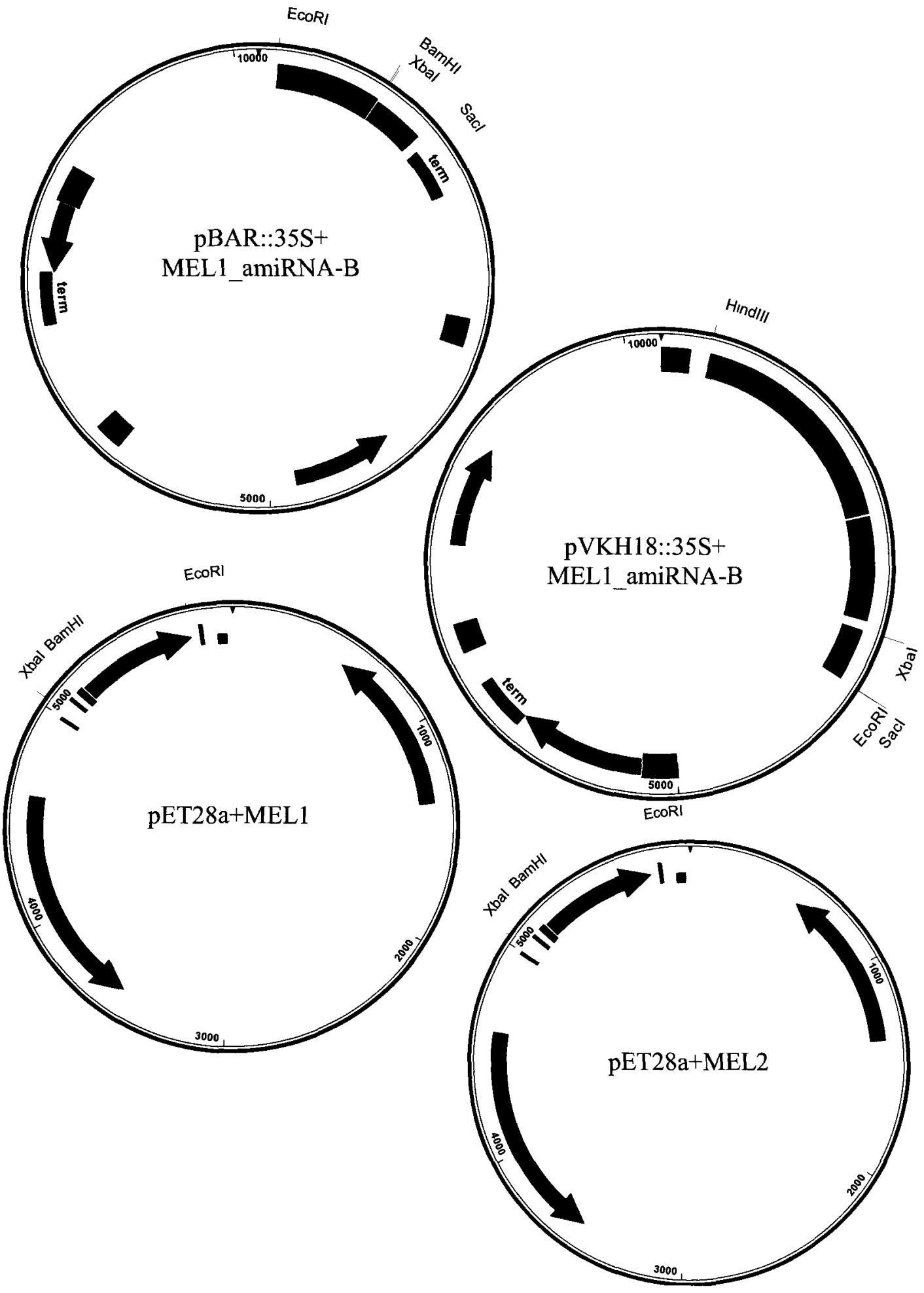




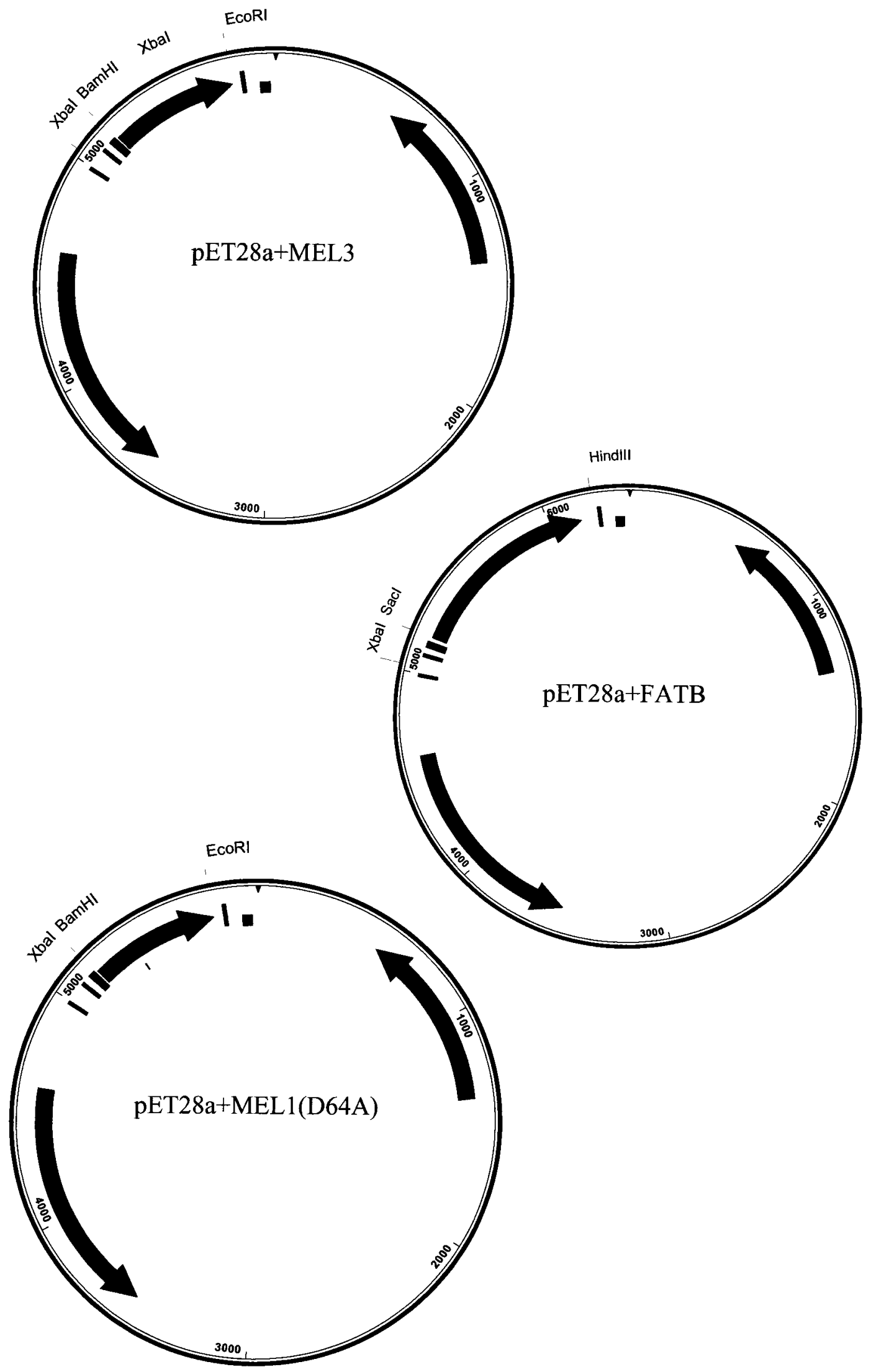


Appendix 3: Previously identified cuticle associated genes.

\begin{tabular}{|c|c|c|c|}
\hline Gene Name & Locus & Function & Reference \\
\hline CERI & Atlg02205 & $\begin{array}{l}\text { Unknown function. Putative } \\
\text { oxidoreductase, } \\
\text { metalloenzyme. }\end{array}$ & $\begin{array}{l}\text { Aarts et al., } 1995, \\
\text { Hannoufa et al, } \\
\text { 1993, McNevin, et } \\
\text { al., 1993. }\end{array}$ \\
\hline CER2 & At4g24510 & $\begin{array}{l}\text { Unknown function. Putative } \\
\text { acyltransferase }\end{array}$ & $\begin{array}{l}\text { Lai et al., } 2007, \\
\text { Negruk et al., } \\
\text { 1996, Xia et al., } \\
\text { 1996, Xia et al., } \\
\text { 1997. }\end{array}$ \\
\hline $\begin{array}{c}C E R 3 \\
(W A X 2 / Y R E / F L P)\end{array}$ & At5g57800 & $\begin{array}{l}\text { Unknown function. Putative } \\
\text { oxidoreductase, } \\
\text { metalloenzyme }\end{array}$ & $\begin{array}{l}\text { Ariizumi } \text { et al., } \\
\text { 2003, Chen et al., } \\
\text { 2003, Kurata } \text { et } \\
\text { al., 2003, Rowland } \\
\text { et al., } 2007\end{array}$ \\
\hline CER4/FAR3 & At4g33790 & $\begin{array}{l}\text { Alcohol forming fatty acyl- } \\
\text { coenzyme A reductase }\end{array}$ & $\begin{array}{l}\text { Rowland } \text { et al., } \\
2006\end{array}$ \\
\hline CER5 (WBC12) & Atlg51500 & $\mathrm{ABC}$ transporter & $\begin{array}{l}\text { Pighin et al., 2004, } \\
\text { Rashotte } \text { et al., } \\
2001 .\end{array}$ \\
\hline $\begin{array}{c}\text { CER6 } \\
\text { (CUT1/KCS6) }\end{array}$ & Atlg68530 & $\begin{array}{l}\beta \text {-ketoacyl-coenzyme A } \\
\text { synthase. (Condensing } \\
\text { enzyme) }\end{array}$ & $\begin{array}{l}\text { Fiebig et al., 2000, } \\
\text { Miller et al., 1999, } \\
\text { Lai et al., 2007. }\end{array}$ \\
\hline CER7 (RRP45) & At3g60500 & Exosome subunit & $\begin{array}{l}\text { Hooker et al, } \\
2007 \text {. }\end{array}$ \\
\hline CER8 (LACS1) & At2g47240 & $\begin{array}{l}\text { Long chain acyl-CoA } \\
\text { synthetase }\end{array}$ & $\begin{array}{l}\text { Lü, et al., } 2009 . \\
\text { Weng et al., } 2010 .\end{array}$ \\
\hline FATB & Atlg08510 & Acyl-ACP thioesterase & $\begin{array}{l}\text { Mayer and } \\
\text { Shanklin, 2005, } \\
\text { Bonaventure et al., } \\
\text { 2003, Salas and } \\
\text { Ohlrogge, } 2002 \text {. }\end{array}$ \\
\hline WSD1 & At5g37300 & $\begin{array}{l}\text { Wax synthase/acyl- } \\
\text { coenzyme A: diacylglycerol } \\
\text { acyltransferase. }\end{array}$ & Li et al., 2008. \\
\hline$M A H I$ & Atlg57750 & $\begin{array}{l}\text { Cytochrome P450 } \\
\text { hydroxylase }\end{array}$ & Greer et al., 2007. \\
\hline$W B C 11$ & Atlg17840 & $\mathrm{ABC}$ transporter & $\begin{array}{l}\text { Bird et al., 2007, } \\
\text { Panikashvili et al., } \\
\text { 2007, Luo et al., } \\
2007 .\end{array}$ \\
\hline$K C S 1$ & Atlg01120 & $\begin{array}{l}\beta \text {-ketoacyl-coenzyme A } \\
\text { synthase. (Condensing } \\
\text { enzyme) }\end{array}$ & Todd et al., 1999. \\
\hline
\end{tabular}




\begin{tabular}{|c|c|c|c|}
\hline$K C S 2(D A I S Y)$ & Atlg04220 & $\begin{array}{l}\beta \text {-ketoacyl-coenzyme A } \\
\text { synthase. (Condensing } \\
\text { enzyme) }\end{array}$ & $\begin{array}{l}\text { Franke } \text { et al., } \\
\text { 2009, Lee } \text { et al., } \\
\text { 2009a. }\end{array}$ \\
\hline$K C S 20$ & At5g 43760 & $\begin{array}{l}\beta \text {-ketoacyl-coenzyme A } \\
\text { synthase. (Condensing } \\
\text { enzyme) }\end{array}$ & Lee et al., 2009a. \\
\hline$P A S 2(H C D)$ & At5g10480 & $\begin{array}{l}\text { 3-hydroxy-acyl-CoA } \\
\text { dehydratase }\end{array}$ & Bach et al., 2008. \\
\hline$K C R I$ & Atlg67730 & $\beta$-ketoacyl-CoA reductase & $\begin{array}{l}\text { Beaudoin et al., } \\
2009 \text {. }\end{array}$ \\
\hline$L A C S 2$ & At1g49430 & $\begin{array}{l}\text { Long chain acyl-CoA } \\
\text { synthetase }\end{array}$ & $\begin{array}{l}\text { Schnurr et al., } \\
\text { 2004, Lü, et al., } \\
\text { 2009, Weng et al., } \\
2010 \text {. }\end{array}$ \\
\hline$L T P G$ & Atlg27950 & $\begin{array}{l}\text { Glycosylphosphatidylinositol } \\
\text {-anchored lipid transfer } \\
\text { protein }\end{array}$ & $\begin{array}{l}\text { Lee } \text { et al., } 2009 \mathrm{~b}, \\
\text { Debono et al., } \\
2009 \text {. }\end{array}$ \\
\hline SHN1 (WIN1) & Atlg15360 & AP2-type transcription factor & $\begin{array}{l}\text { Aharoni et al., } \\
\text { 2004, Kannangara } \\
\text { et al., 2007, Broun } \\
\text { et al., } 2004 .\end{array}$ \\
\hline SHN2 & At5g25390 & AP2-type transcription factor & $\begin{array}{l}\text { Aharoni et al., } \\
2004 .\end{array}$ \\
\hline SHN3 & At5g11190 & AP2-type transcription factor & $\begin{array}{l}\text { Aharoni et al., } \\
2004 .\end{array}$ \\
\hline MYB30 & At3g28910 & MYB transcription factor & $\begin{array}{l}\text { Raffaele } \text { et al., } \\
2008 \text {. }\end{array}$ \\
\hline$M Y B 41$ & At4g28110 & MYB transcription factor & $\begin{array}{l}\text { Lippold et al., } \\
2009 \text {. }\end{array}$ \\
\hline ATT1 & At4g00360 & $\begin{array}{l}\text { Cytochrome P450 } \\
\text { monooxygenase }\end{array}$ & Xiao et al., 2004. \\
\hline$L C R$ & At2g45970 & $\begin{array}{l}\text { Cytochrome P450 } \\
\text { monooxygenase }\end{array}$ & $\begin{array}{l}\text { Wellesen et al., } \\
2001 \text {. }\end{array}$ \\
\hline GPAT4 & Atlg01610 & $\begin{array}{l}\text { Glycerol-3-phosphate } \\
\text { acyltransferase }\end{array}$ & Li et al., 2007. \\
\hline GPAT8 & At4g00400 & $\begin{array}{l}\text { Glycerol-3-phosphate } \\
\text { acyltransferase }\end{array}$ & Li et al., 2007. \\
\hline$D C R$ & At5g23940 & BAHD acyltransferase & $\begin{array}{l}\text { Panikashvili et al., } \\
2009 .\end{array}$ \\
\hline
\end{tabular}


Appendix 4: Previously identified suberin associated genes.

\begin{tabular}{|c|c|c|c|}
\hline Gene Name & Locus & Enzyme & Reference \\
\hline GPAT5 & At3g11430 & $\begin{array}{l}\text { Glycerol-3-phosphate } \\
\text { acyltransferase }\end{array}$ & Beisson et al., 2007. \\
\hline CYP86A1 & At5g58860 & Fatty acid hydroxylase. & $\begin{array}{l}\text { Li } \text { et al., 2007. Höfer et } \\
\text { al., 2008. }\end{array}$ \\
\hline CYP86B1 & At5g23190 & cytochrome P450 & \\
\hline $\begin{array}{c}K C S 2 \\
(D A I S Y)\end{array}$ & Atlg04220 & $\begin{array}{l}\text { B-ketoacyl-coenzyme A } \\
\text { synthase. (Condensing } \\
\text { enzyme) }\end{array}$ & $\begin{array}{l}\text { Franke } \text { et al., 2009, Lee } \\
\text { et al., 2009a. }\end{array}$ \\
\hline$K C S 20$ & At5g43760 & $\begin{array}{l}\beta \text {-ketoacyl-coenzyme A } \\
\text { synthase. (Condensing } \\
\text { enzyme) }\end{array}$ & Lee et al., 2009a. \\
\hline FARI & At5g22500 & $\begin{array}{l}\text { Alcohol forming fatty acyl- } \\
\text { coenzyme A reductase }\end{array}$ & Domergue et al., 2010. \\
\hline FAR4 & At3g44540 & $\begin{array}{l}\text { Alcohol forming fatty acyl- } \\
\text { coenzyme A reductase }\end{array}$ & Domergue et al., 2010. \\
\hline FAR5 & At3g44550 & $\begin{array}{l}\text { Alcohol forming fatty acyl- } \\
\text { coenzyme A reductase }\end{array}$ & Domergue et al., 2010. \\
\hline$A F S T$ & At5g41040 & Feruloyl-CoA transferase & $\begin{array}{l}\text { Molina et al., } 2009, \text { Gao } \\
\text { et al., } 2009 .\end{array}$ \\
\hline ESB1 & At2g28670 & Unknown function & Baxter et al., 2009. \\
\hline $\begin{array}{c}\text { CER8 } \\
(L A C S 1)\end{array}$ & At2g47240 & $\begin{array}{l}\text { Long chain acyl-CoA } \\
\text { synthetase }\end{array}$ & $\begin{array}{l}\text { Lü, et al., 2009, Weng et } \\
\text { al., 2010, Li - Beisson et } \\
\text { al., 2010. }\end{array}$ \\
\hline$L A C S 2$ & Atlg49430 & $\begin{array}{l}\text { Long chain acyl-CoA } \\
\text { synthetase }\end{array}$ & $\begin{array}{l}\text { Schnurr et al., 2004, Lü, } \\
\text { et al., 2009, Weng et al., } \\
2010, \text { Li-Beisson et al, } \\
2010 .\end{array}$ \\
\hline
\end{tabular}




\section{Appendix 5: Amino acid sequence alignment of the predicted MEL proteins with related single-domain Hotdog fold proteins.}

Predicted Hotdog fold plant thioesterases from Arabidopsis thaliana (At) Arabidopsis lyrata $(\mathrm{Al})$, Chlamydomomas reinhardtii $(\mathrm{Cr})$, Ricinus communis $(\mathrm{Rc})$, Solanum habrochaites $(\mathrm{Sh})$, Zea mays $(\mathrm{Zm})$, Oryza sativa $(\mathrm{Os})$, Vitis vinifera $(\mathrm{Vv})$, Amborella trichopoda (Atr), Picea glauca (Pg), Humulus lupulus (H1), Petunia integrifolia (Pi), Solanum lycopersicum (S1), Lycopersicon hirsutum (Lh), Picea sitchensis (Ps), Selaginella moellendorfii (Sm), and Gossypium hirsutum (hㅡ). In this alignment, conserved amino acids are highlighted in black while similar amino acids are highlighted in gray. The MEL proteins contain the conserved catalytic residues (Asp 64, Gly 67, and Val 69 in MEL1) observed in the 4-hydroxybenzoyl CoA thioesterase (4HBT) from Pseudomonas (Benning et al., 1998). Across all sequences the catalytic aspartic acid, noted with *, has $100 \%$ conservation while the glycine and valine, noted with \#, are conserved in $95 \%$ of sequences, including MELs. In 4HBT, a tyrosine residue is predicted to involved with nucleophilic attack, however, this predicted tyrosine is not conserved in plant thioesterases. It is possible that the tyrosine residue two amino acids downstream (noted with an @) may carryout this function. Nomenclature is as follows: species name_accession number. 
At lg35290 (MEL1) At Ig35250 (MEL2) At lg68260 (MEL3) At lg68280 (MEL4) Al-XP 002893861 Al-XP 002891170 $\mathrm{Al}-\mathrm{XP} 002893863$ Al-XP 002888651 Sh-MKS̄II

RC-XP 002526988 $\mathrm{VV}-\mathrm{CBI} 27033$

S1-ACG69783

Pi-AAS 90598

Lh-ACG63705

PS-ADE77741

Os-CAE01692

Os-CAD4122

Os-BAD25745

Os-BAD2 5619

Zm-NP 001183748

Sm-XP 002968328

$\mathrm{Sb}-\mathrm{XP}-002448292$

$\mathrm{Sb}-\mathrm{XP} \quad 002452801$

$\mathrm{Sb}-\mathrm{XP}-002452802$

Atr-FD440753

$\mathrm{Pg}-\mathrm{Ex} 412733$

H1-GD2 49868

Gh-DT554179

Cr-XP 001703093

Pseudōmonas-4HBT

At 1 g35290 (MEL1) At 1g35250 (MEL2) At 1g68260 (MEL3) At 1 g68280 (MEL4) $\mathrm{Al}-\mathrm{XP} \quad 002893861$ $\mathrm{Al}-\mathrm{XP}-002891170$ $\mathrm{Al}-\mathrm{XP} \quad 002893863$ Al-XP_002888651 Sh-MKS̄II

RC-XP 002526988

$\mathrm{VV}-\mathrm{CB} \overline{\mathrm{I}} 27033$

SI-ACG 69783

Pi-AAS 90598

Lh-ACG63705

Ps-ADE 77741

Os-CAE01692

Os-CAD 4122

Os-BAD25745

Os-BAD25619

Zm-NP 001183748

Sm-XP 002968328

$\mathrm{Sb}-\mathrm{XP} 002448292$

$\mathrm{Sb}-\mathrm{XP} 002452801$

$\mathrm{Sb}-\mathrm{XP}-002452802$

Atr-FD 440753

$\mathrm{Pg}-\mathrm{Ex} 412733$

H1-GD2 49868

Gh-DT55 4179

Cr-XP 001703093

Pseudomonas-4HBT
1 -- - - - - - MLKATGTVAPAM---HVVFPCFSS--------RPI

1 - - - - - MFQATSTGAQIM---HAAFPRSWR--------RGH

- - - - -MFLQV-TGTATPAMP--AVVFLNSWR-------RPL

- - - - - - MIRVTGTAAPAM---SVVFPTSWR--------QPV

----------MFQATGMAAPVM---HVVFPRSWR--------RRH

- - - - - - MFQATGRAAPAM-- HVMFPCSSR--.-----RPV

- - - - - MFQAAATAAQAMPVQHVGFPRFLS-.-- - - RQI

--------MFQITGTGTAAPAM---SMVFPNSSR--------RPV

-- - - - - MSHSFSIA-TNITLLNHGSPPSTEPVIPHRQLPLPNL

-MALQQAF IYPMQVTTPLSRANTTWINLHRPSASLLFRVSRPP---MSPV

---------MAVPASRADTRGIRLYCP--PLILPAPQPPSNCRSPR

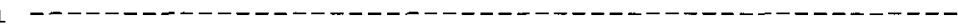

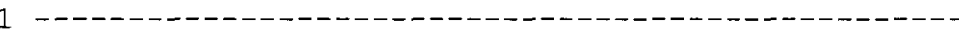

1 - - - - - -

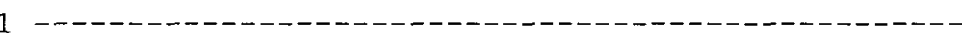

- - - - MHHQIWRLLPSA-- ISPIHAGAPRPSRPPARLGRPSPQR - - - - MQQQQLCSSHCLPARAGS IASPGSGRRVVPLGRRRASLGKV --------MQQPSVGIVVPNACSHPRNDAVPRAGSSRSR----RSSL - - - - MQQPSVS-LAPNTSCHPQHAGSAAGSSRRSHG---HLGV - - - - MHHRFAGLVPTARPAIPPIHGGVVGRSYP--PVHRSLALR

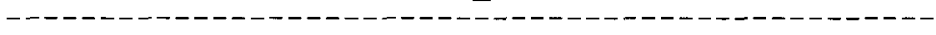
- - - - MHHQFARLVPTARPAIPPIHGGAVGRSSP--HVHRAVALR - ---MEFCAQQQLFNPPPPPPSTTKRCPSPAAPSSTARASWPRPRHPAV ------MITPLAGFSGRPAEPG---REVCLLQPCQASKAER------QV -----MQATWSQSVQCLAFPGRAPMAHVANNKPPHLRFSLFNPNRSPSS MATAMGA ISGGISVGVNARYPHVQCSSFIONPTKKLSRALAFPSLRTASC - - - - --MLQTFSPSYKPLHLPISSLSLSSFS SSSASSVAFPVTRL ---MLQASVFPAHAALPSPRPNATFINLHRPSSSFPIS-----PLLMPLR

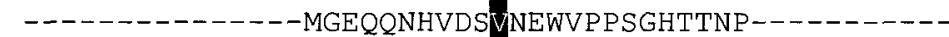

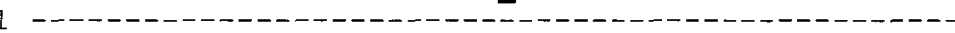

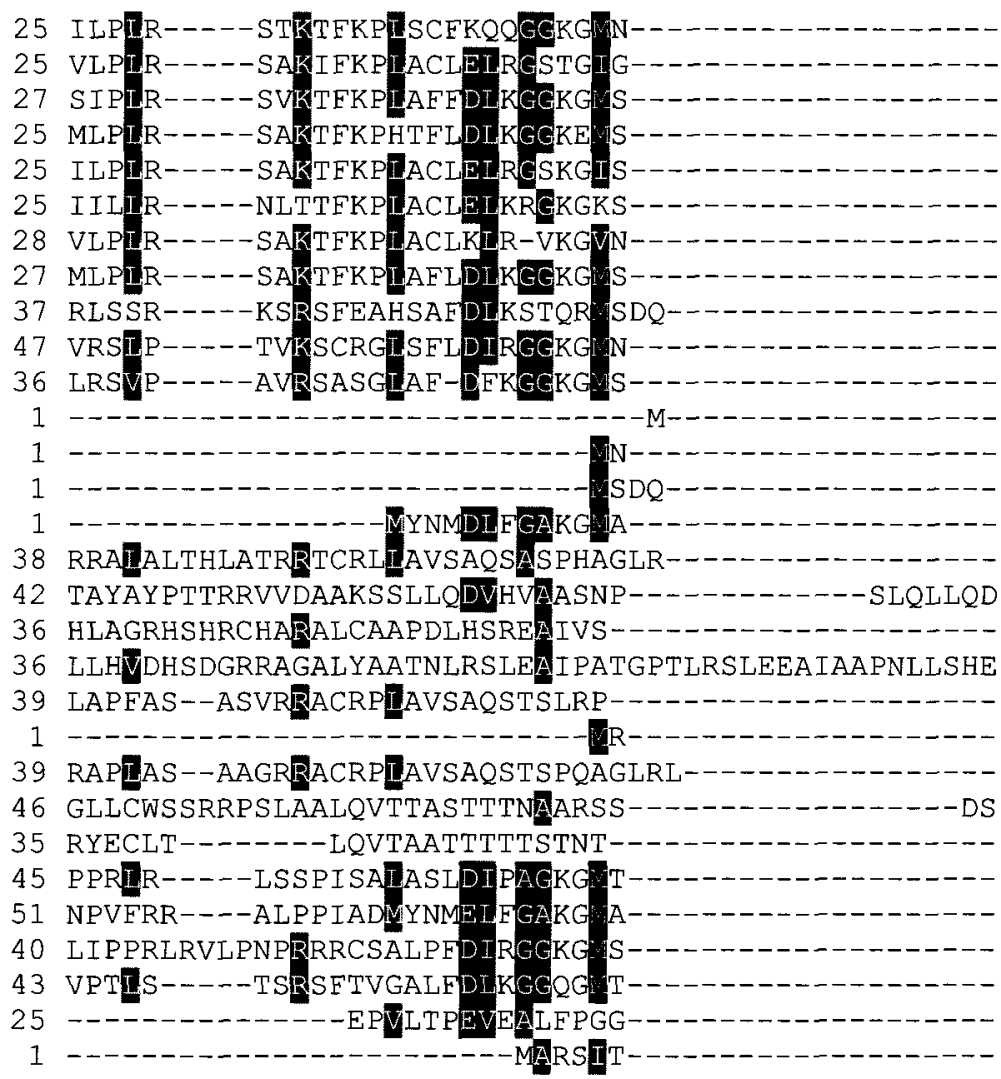


At1g35290 (MEL1) At1g35250 (MEL2) At1g68260 (MEL3) At 1 g68280 (MEL4) Al-XP 002893861 $\mathrm{Al}-\mathrm{XP}-002891170$ $\mathrm{Al}-\mathrm{XP}-002893863$ Al-XP-002888651 Sh-MKS̄II

Rc-XP_002526988

Vv-CBĪ 27033

SI-ACG69783

Pi-AAS 90598

Lh-ACG63705

Ps-ADE 77741

OS-CAE01692

OS-CAD 4122

Os-BAD25745

Os-BAD25619

Zm-NP 001183748

Sm-XP 002968328

$\mathrm{Sb}-\mathrm{XP}-002448292$

$\mathrm{Sb}-\mathrm{XP}-002452801$

$\mathrm{Sb}-\mathrm{XP} 002452802$

Atr-FD 440753

Pg-Ex412733

H1-GD2 49868

Gh-DT554179

Cr-XP_001703093

Pseudōmonas-4HBT

At 1g35290 (MEL1) At Ig35250 (MEL2) At 1968260 (MEL3) At 1968280 (MEL4) Al-XP 002893861 Al-XP_002891170 Al-XP 002893863 Al-XP_002888651 Sh-MKS I I

$\mathrm{RC}-\mathrm{XP} \quad 002526988$

$\mathrm{VV}-\mathrm{CBI} 27033$

SI-ACG 69783

Pi-AAS 90598

Lh-ACG63705

PS-ADE77741

OS-CAE01692

Os-CAD4122

Os-BAD25745

Os-BAD2 5619

Zm-NP_001183748

Sm-XP 002968328

$\mathrm{Sb}-\mathrm{XP}-002448292$

$\mathrm{Sb}-\mathrm{XP} \quad 002452801$

$\mathrm{Sb}-\mathrm{XP} 002452802$

Atr-FD 440753

Pg-Ex412733

H1-GD249868

Gh-DT554179

Cr-XP_001703093

Pseudomonas-4HBT
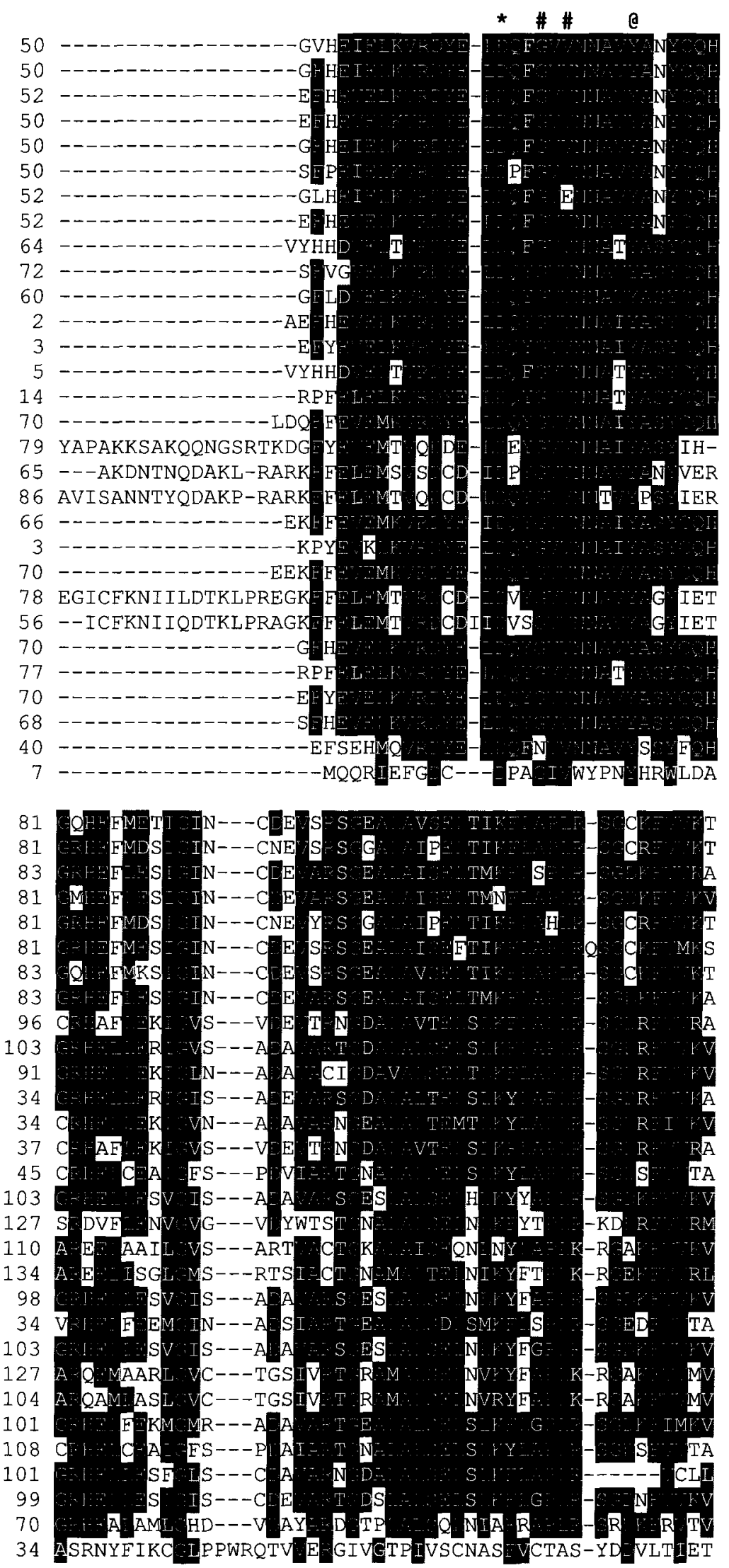
Atlg35290 (MEL1) At 1g35250 (MEL2) At lg68260 (MEL3) At lg68280 (MEL4) A1-XP 002893861 $A 1-X P \quad 002891170$ $\mathrm{A} 1-\mathrm{XP}-002893863$ A1-XP 002888651 Sh-MKS I I

Rc-XP 002526988 $\mathrm{Vv}-\mathrm{CB} \overrightarrow{\mathrm{I}} 27033$

S1-ACG69783

Pi-AAS 90598

In-ACG 63705

Ps-ADE 77741

Os-CAE01692

Os-CAD4122

Os-BAD25745

Os-BAD2 5619

Zm-NP 001183748

Sm-XP 002968328

$\mathrm{Sb}-\mathrm{XP}-002448292$

$\mathrm{Sb}-\mathrm{XP} \quad 002452801$

$\mathrm{Sb}-\mathrm{XP}-002452802$

At $r-F \bar{D} 440753$

$\mathrm{Pg}-\mathrm{Ex} 412733$

H1-GD2 49868

Gh-DT554179

Cr-XP 001703093

Pseudōmonas-4HBT

At 1g35290 (MEL1) At Ig35250 (MEL2) At 1g68260 (MEL3) At 1968280 (MEL4) Al-XP 002893861 $A 1-X P-002891170$ $\mathrm{Al}-\mathrm{XP}-002893863$ Al-XP_002888651 Sh-MKS̄II

RC-XP 002526988

$\mathrm{Vv}-\mathrm{CB} \overline{\mathrm{I}} 27033$

Sl-ACG 69783

Pi-AAS 90598

Lh-ACG63705

PS-ADE 77741

Os-CAE01692

Os-CAD 4122

Os-BAD25745

Os-BAD2 5619

Zm-NP 001183748

Sm-XP 002968328

$\mathrm{Sb}-\mathrm{XP}-002448292$

$\mathrm{Sb}-\mathrm{XP}-002452801$

$\mathrm{Sb}-\mathrm{XP}-002452802$

Atr-FD 440753

$\mathrm{Pg}-\operatorname{Ex} 412733$

H1-GD249868

Gh-DT554179

Cr-XP 001703093

Pseudomonas-4HBT

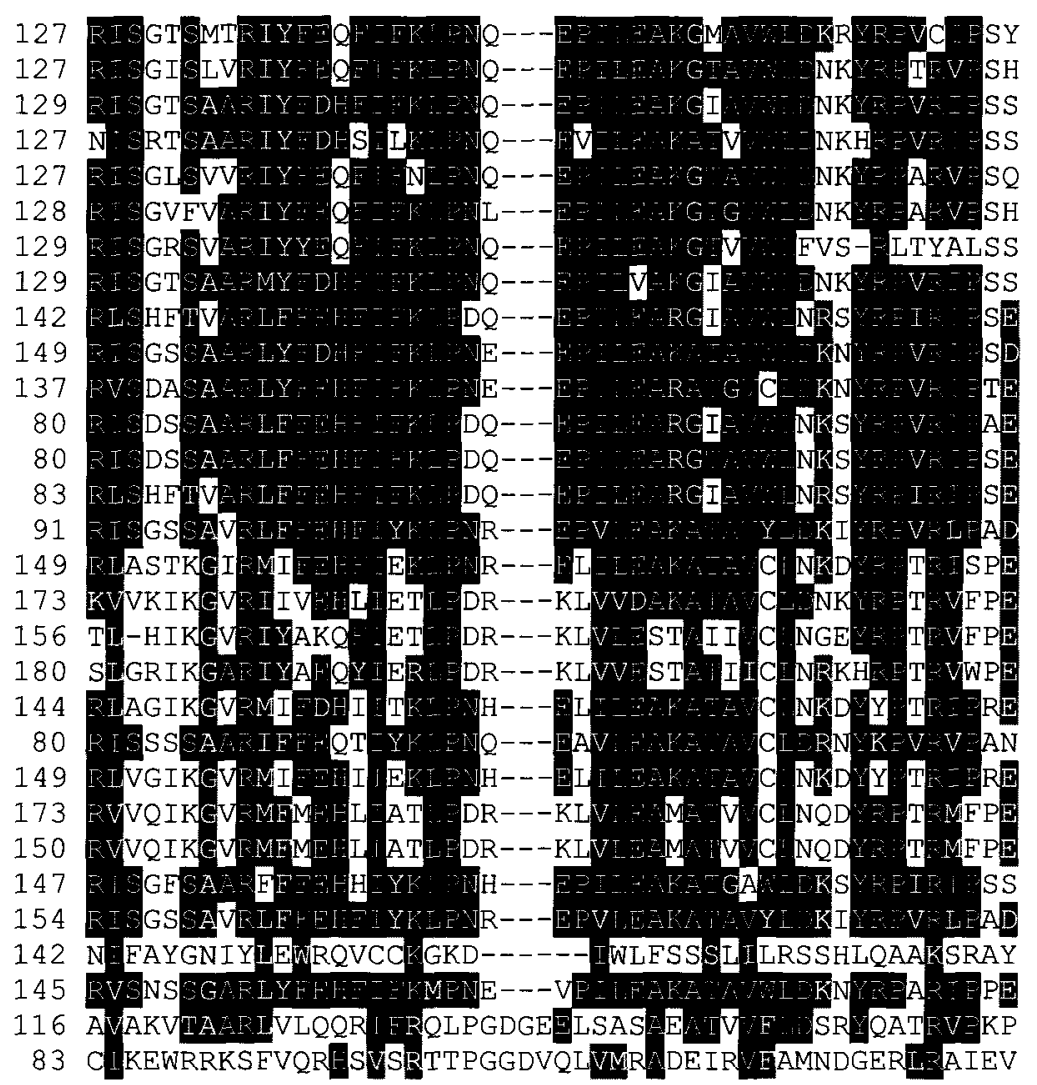

119 
Appendix 6: Types of aliphatic compounds in the first and second generations of MELI amiRNA and MEL1-35S.

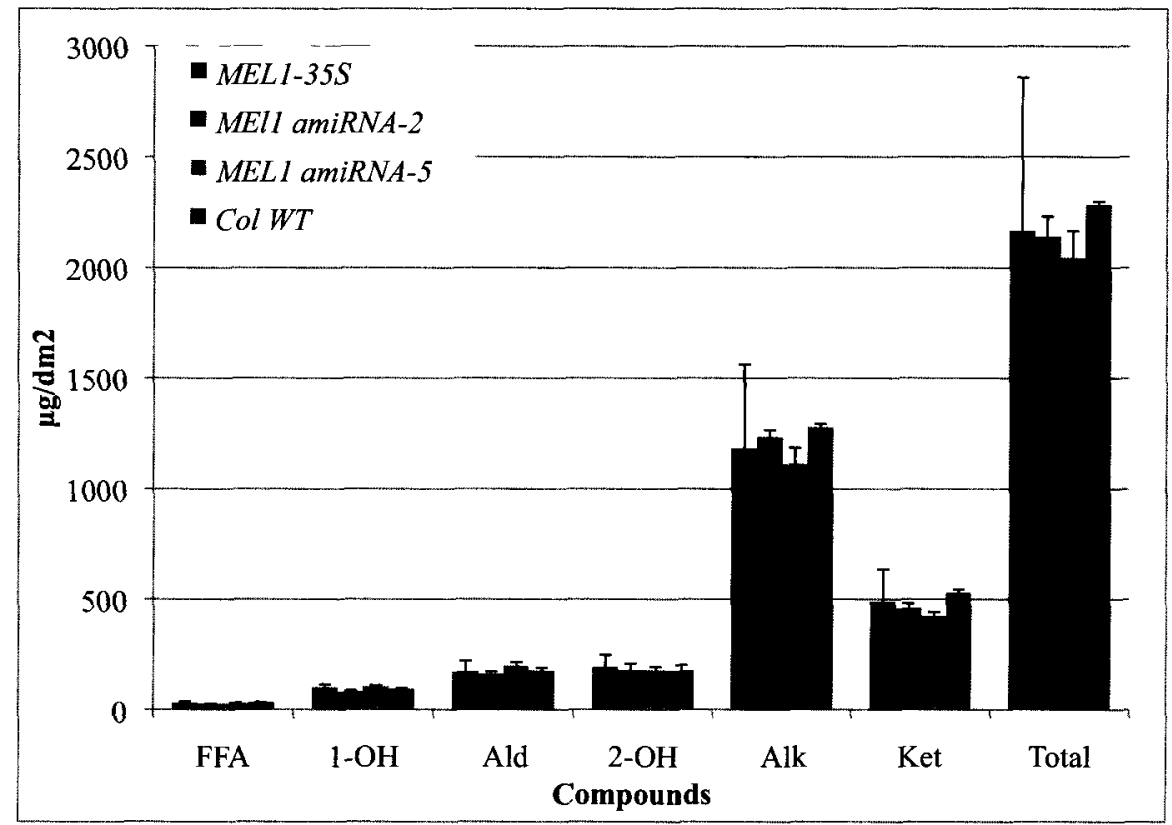

Figure Appendix 6A: Types of aliphatic compounds found in the cuticular waxes in the T2 generation as determined by GC-FID.

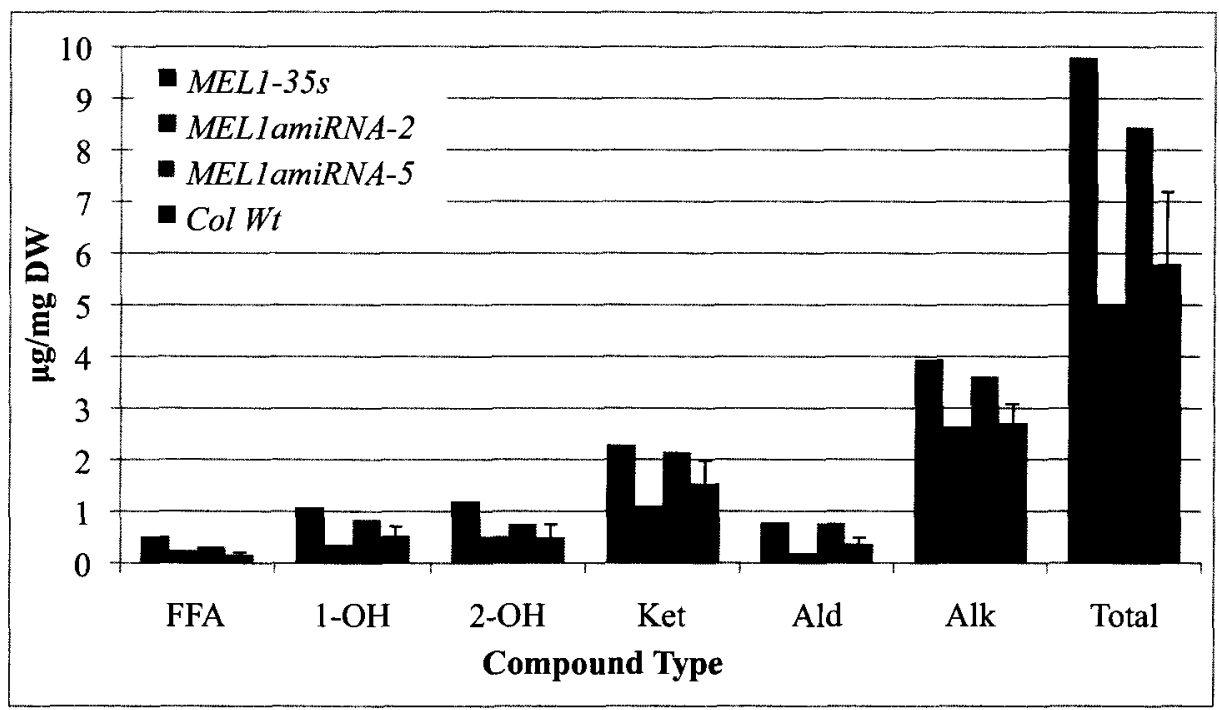

Figure Appendix 6B: Types of aliphatic compounds found in the cuticular waxes in the T1 generation of plants as determined by GC-FID. 


\section{References}

1. Aarts, M. G. M., Keijzer, C. J., Stiekema, W. J., and Pereira, A. 1995. Molecular characterization of the CERI gene of Arabidopsis involved in epicuticular wax biosynthesis and pollen fertility. Plant Cell. 7: 2115-2127.

2. Aarts, M. G., Hodge, R., Kalantidis, K., Florack, D., Wilson, Z. A., Mulligan, B. J., Stiekema, W. J., Scott, R., and Pereira, A. 1997. The Arabidopsis MALE STERILITY 2 protein shares similarity with reductases in elongation/condensation complexes. Plant Journal. 12: 615-623.

3. Aharoni, A., Dixit, S., Jetter, R., Thoenes, E., van Arkel, G., and Pereira, A. 2004. The SHINE clade of AP2 domain transcription factors activates wax biosynthesis, alters cuticle properties, and confers drought tolerance when overexpressed in Arabidopsis. Plant Cell. 16: 2463-2480.

4. Ahlers, F., Thom, I., Lambert, J., Kuckuk, R., and Wiermann, R. 1999. ' H NMR analysis of sporopollenin from Typha Angustifolia. Phytochemistry. 50: 10951098.

5. Ariizumi, T., Hatakeyama, K., Hinata, K., Sato, S., Kato, T., Tabata, S., and Toriyama, K. 2003. A novel male-sterile mutant of Arabidopsis thaliana, faceless pollen-1, produces pollen with a smooth surface and an acetolysis-sensitive exine. Plant Molecular Biology. 53: 107-116.

6. Arnold, K., Bordoli, L., Kopp, J., and Schwede, T. 2006. The SWISS-MODEL Workplace: A web-based environment for protein structure homology modeling. Bioinformatics. 22: 195-201. 
7. Bach, L., Michaelson, L. V., Haslam, R., Bellec, Y., Gissot, L., Marion, J., Da costa, M., Boutin, J. P., Miquel, M., Tellier, F., Domergue, F., Markham, J. E., Beaudoin, F., Napier, J. A., and Faure, J. D. 2008. The very-long-chain hydroxy fatty acyl-CoA dehydratase PASTICCINO2 is essential and limiting for plant development. PNAS. 105: 14727-14731.

8. Batoko, H., Zheng, H.-Q., Hawes, C., and Moore, I. 2000. A Rab1 GTPase is required for transport between the endoplasmic reticulum and golgi apparatus and for normal golgi movement in plants. The Plant Cell. 12: 2201-2217.

9. Baxter, I., Hosmani, P. S., Rus, A., Lahner, B., Borevitz, J. O., Muthukumar, B., Mickelbart, M. V., Schreiber, L., Franke, R. B., and Salt, D. E. 2009. Root suberin forms an extracellular barrier that affects water relations and mineral nutrition in Arabidopsis. PLoS Genetics. 5: e1000492.

10. Beaudoin, F., Wu, X., Li, F., Haslam, R. P., Markham, J. E., Zheng, H., Napier, J. A., and Kunst, L. 2009. Functional characterization of the Arabidopsis betaketoacyl-coenzyme A reductases candidates of the fatty acid elongase. Plant Physiology. 150: 1174-1191.

11. Beisson, F., Li, Y., Bonaventure, G., Pollard, M., and Ohlrogge, J. B. 2007. The acyltransferase GPAT5 is required for the synthesis of suberin in seed coat and root of Arabidopsis. Plant Cell. 19: 351-368.

12. Ben-Israel, I., Yu, G., Austin, M. B., Bhuiyan, N., Auldridge, M., Nguyen, T, Schauvinhold, I., Noel, J. P., Pichersky, E., and Fridman, E. 2009. Multiple biochemical and morphological factors underlie the production of methylketones in tomato trichomes. Plant Physiology. 151: 1952-1964. 
13. Beisson, F., Li, Y., Bonaventure, G., Pollard, M., and Ohlrogge, J. B. 2007. The Acyltransferase GPAT5 is required for the synthesis of suberin in seed coat and root of Arabidopsis. The Plant Cell. 19: 351-368.

14. Benning, M. M., Wesenberg, G., Liu, R., Taylor, K. L., Donaway-Mariano, D., and Holden, H. M. 1998. The three-dimensional structure of 4- hydroxybenzoyl-CoA thioesterase from Pseudomonas sp. Strain CBS-3. Journal of Biological Chemistry. 273: $33572-22579$.

15. Benveniste, I., Tijet, N., Adas, F., Philipps, G., Salaün, J.P. and Durst, F. 1998. CYP86A1 from Arabidopsis thaliana encodes a cytochrome P450-dependent fatty acid omega-hydroxylase. Biochemical and Biophysical Research Communications. 243: 688-693.

16. Benveniste, I., Saito, T., Wang, Y., Kandel, S., Huang, H., Pinot, F., Kahn, R. A., Salaün, J.P., and Shimoji, M. 2006. Evolutionary relationship and substrate specificity of Arabidopsis thaliana fatty acid omega-hydroxylase. Plant Science. 170: $326-338$.

17. Bernards, M. A. 2002. Demystifying suberin. Canadian Journal of Botany. 80: $227-240$.

18. Bird, D., Beisson, F., Brigham, A., Shin, J., Greer, S., Jetter, R., Kunst, L., Wu, X., Yephremov, A., and Samuels, L. 2007. Characterization of Arabidopsis $\mathrm{ABCG11/WBC11}$, an ATP binding cassette $(\mathrm{ABC})$ transporter that is required for cuticular lipid secretion. Plant Journal. 52: 485-489. 
19. Bonaventure, G., Salas, J. J., Pollard, M. R., and Ohlrogge, J. B. 2003. Disruption of the FATB gene in Arabidopsis demonstrates an essential role of saturated fatty acids in plant growth. Plant Cell. 15: 1020-1033.

20. Bonaventure, G., Beisson, F., Ohlrogge, J., and Pollard, M. 2004. Analysis of the aliphatic monomer composition of polyesters associated with Arabidopsis epidermis: occurrence of octadeca-cis-6, cis-9-diene-1, 18-dioate as the major component. The Plant Journal. 40: 920-930.

21. Broun P, Poindexter P, Osborne E, Jiang C-Z and Riechmann JL. 2004. WIN1, a transcriptional activator of epidermal wax accumulation in Arabidopsis. Proceedings of the National Academy of Sciences of the United States of America. 101: $4706-4711$.

22. Buchanan, B. B., Gruissem, W., and Jones, R. 2000. Lipids. In Biochemistry and Molecular Biology of Plants. American Society of Plant Biologists. 456-527.

23. Cantu, D. C., Chen, Y., and Reilly, P. J. 2010a. ThYme: A database for thioesteractive enzymes. Nucleic Acids Research. doi: 10.1093/nar/gkq1072.

24. Cantu, D. C., Chen, Y., and Reilly, P. J. 2010b. Thioesterases: A new perspective based on their primary and tertiary structures. Protein Science. 19: 1281-1295.

25. Cheesborough, T. M. and Kolattukudy, P. E. 1984. Alkane biosynthesis by decarbonylation of aldehydes catalyzed by a particulate preparation from Pisum sativum. Proceedings of the National Academy of Sciences. 81: 6613-6617.

26. Chen, X., Goodwin, S. M., Boroff, V. L., Liu, X., and Jenks, M. A. 2003. Cloning and characterization of the WAX2 gene of Arabidopsis involved in cuticle membrane and wax production. Plant Cell. 15: 1170-1185. 
27. Coen, E. S., Romero, J. M., Doyle, S., Elliot, R., Murphy, G., and Carpenter, R. 1990. Floricaula: A homeotic gene required for flower development in Antirrhinum majus. Cell. 63: 1311-1322.

28. Compagnon, V., Diehl, P., Benveniste, I., Meyer, D., Schaller, H., Schreiber, L, Franke, R., and Pinot, F. 2009. CYP86B1 Is Required for Very Long Chain $\omega-$ Hydroxyacid and $\alpha, \omega$-Dicarboxylic Acid Synthesis in Root and Seed Suberin Polyester. Plant Physiology. 150: 1831-1843.

29. Debono, A., Yeats, T. H., Rose, J. K., Bird, D., Jetter, R., Kunst, L., and Samuels, L. 2009. Arabidopsis LTPG is a glycosylphosphatidylinositol-anchored lipid transfer protein required for export of lipids to the plant surface. Plant Cell. 12: $1230-1238$.

30. Desvergne, B. and Wahli, W. 1999. Peroxisome proliferator-activated receptors: nuclear control of metabolism. Endocrine Reviews. 20: 649-688.

31. Dillon, S. C., and Bateman, A. 2004. The hotdog fold: wrapping up a superfamily of thioesterases and dehydratases. BMC Bioinformatics. 5: 109.

32. Doan, T.T., Carlesson, A.S., Hamberg, M., Bülow, L., Stymne, S., \& Olsson, P. 2009. Functional expression of five Arabidopsis fatty acyl-CoA reductase genes in Escherichia coli. Journal of Plant Physiology. 166: 787-796.

33. Domergue, F., Vishwanath, S. J., Joubès, J., Ono, J., Lee, J. A., Bourdon, M., Alhattab, R., Lowe, C., Pascal, S., Lessire, R., and Rowland O. 2010. Three Arabidopsis fatty acyl-Coenzyme A reductases, FAR1, FAR4, and FAR5, generate primary fatty alcohols associated with suberin deposition. Plant Physiology. 153: 1-16. 
34. Dominguez, E., Mercado, J.A., Quesada, M.A., and Heredia, A. 1999. Pollen sporopollenin: degradation and structural elucidation. Sexual Plant Reproduction. 12: $171-178$.

35. Elholm, M., Dam, I., Jorgensen, C., Krogsdam, A-M., Holst, D., Kratchmarova, I., Gottlicher, M., Berge, R., Flatmark, T., Knudsen, J., Mandrup, S., and Kristiansen, K. 2001. Acyl-CoA esters antagonize the effects of ligands on peroxisome proliferator-activated receptor $\alpha$ conformation, DNA binding, and interactions with Co-factors. The Journal of Biological Chemistry. 276: 21410-21416.

36. Ellman, G. L. 1958. A colorimetric method for determining low concentrations of mercatans. Archives Biochemistry and Biophysics. 75: 443-450.

37. Fehling, E., and Mukherjii, K. D. 1991. Acyl-CoA enlongase from a higher plant (Lunaria annua): metabolic intermediates of very-long-chain acyl-CoA products and substrate specificity. Biochimica et Biophysica Acta. 1082: 239-246.

38. Fiebig, A., Mayfield, J. A., Miley, N. L., Chau, S., Fischer, R. L., and Preuss, D. 2000. Alterations in CER6, a gene identical to CUT1, differentially affect longchain lipid content on the surface of pollen and stems. Plant Cell. 12: 2001-2008.

39. Franke, R., Briesen, I., Wojciechowski, T., Faust, A., Yephremov, A., Nawrath, C., and Schreiber, L. 2005. Apoplastic polyesters in Arabidopsis surface tissues-A typical suberin and a particular cutin. Phytochemistry. 66: 2643-2658.

40. Franke, R. and Schreiber, L. 2007. Suberin-a biopolyester forming apoplastic plant interfaces. Current Opinion in Plant Biology. 10: 252-259.

41. Franke, R., Höfer, R., Briesen, I., Emsermann, M., Efremova, N., Yphremov, A., and Schreiber, L. 2009. The DAISY gene from Arabidopsis encodes a fatty acid 
elongase condensing enzyme involved in the biosynthesis of aliphatic suberin in roots and the chalaza-micropyle region of seeds. Plant Journal. 57: 80-95.

42. Fridman, E., Wang, J., Iijima, Y., Froehlich, J. E., Gang, D. R., Ohlrogge, J., and Pichersky, E. 2005. Metabolic, genomic, and biochemical analyses of glandular trichomes from the wild tomato species Lycopersicon hirsutum identify a key enzyme in the biosynthesis of methylketones. The Plant Cell. 17: 1252-1267.

43. Fujita, Y., Matsuoka, H., and Hirooka, K. 2007. Regulation of fatty acid metabolism in bacteria. Molecular Microbiology. 66: 829-839.

44. Gao, J., Ajjawi, I., Manoli, A., Sawin, A., Xu, C., Froehlich, J.E., Last, R.L., and Benning, C. 2009. FATTY ACID DESATURASE4 of Arabidopsis encodes a protein distinct from characterized fatty acid desaturases. Plant Journal. 60: 832839.

45. Graça, J. and Santos, S. 2007. Suberin: A Biopolyester of Plant's Skin. Macromolecular Bioscience. 7: 128-135.

46. Graç, J., and Lamosa, P. 2010. Linear and branched poly( $\omega$-hydroxyacid) esters in plant cutins. Journal of Agricultural and Food Chemistry. 58: 9666-9674.

47. Greer, S., Wen, M., Bird, D., Wu, X., Samuels, L., Kunst, L., and Jetter, R. 2007. The cytochrome P450 enzyme CYP96A15 is the midchain alkane hydroxylase responsible for formation of secondary alcohols and ketones in stem cuticular wax of Arabidopsis. Plant Physiology. 145: 653-667.

48. Guilford, W. J., Schneider, D. M., Labovitz, J., and Opella, S. J. 1988. High resolution solid state ${ }^{13} \mathrm{C}$ NMR spectroscopy of Sporopollenins from different plant taxa. Plant Physiology. 86: 134-136. 
49. Hannoufa, A., McNevin, J., and Lemieux, B. 1993. Epicuticular waxes of eceriferum mutants of Arabidopsis thaliana. Phytochemistry. 33: 851-855.

50. Hartmann, K., Peiter, E., Koch, K., Schubert, S., and Schreiber, L. Chemical composition and ultrastructure of broad bean (Vicia faba L.) nodule endodermis in comparison to the root endodermis. Planta. 215: 14-25.

51. Heckman, K.L. and Pease, L.R. 2007. Gene splicing and mutagenesis by PCRdriven overlap extension. Nature Protocols. 2: 924-932

52. Heredia, A. 2003. Biophysical and Biochemical characteristics of cutin, a plant barrier biopolymer. Biochemcia et Biophysica Acta. 1620: 1-7.

53. Hertz, R., Magehheim, J., Berman, I., and Bar-Tana, J. 1998. Fatty acyl-CoA thioesters are ligands of hepatic nuclear factor-4alpha. Nature. 392: 512-516.

54. Höfer, R., Briesen, I., Beck, M., Pinot, F., Schreiber, L., and Franke, R. 2008. The Arabidopsis cytochrome P450 CYP86Al encodes a fatty acid omega-hydroxylase involved in suberin monomer biosynthesis. Journal of Experimental Botany. 59: 2347-2360.

55. Hooker, T. S., Miller, A. A., and Kunst., L. 2002. Significance of the expression of the CER6 condensing enzyme for cuticular wax production in Arabidopsis. Plant Physiology. 129: 1568-1580.

56. Hooker, T. S., Lam, P., Zheng, H., and Kunst, L. 2007. A core subunit of the RNA-processing/degrading exosome specifically influences cuticular wax biosynthesis in Arabidopsis. Plant Cell. 19: 904-913. 
57. Hsieh, K., and Huang, A.H.C. 2007. Tapetosomes in Brassica tapetum accumulate endoplasmic reticulum-derived flavonoids and alkanes for delivery to the pollen surface. The Plant Cell. 19: 582-596.

58. Huijser, P., Klein, J., Lonnig, W. E., Meijer, H., Saedler, H., and Sommer, H. 1992. Bracteomania, an inflorescence anomaly, is caused by the loss of function of the MADS-box gene saquamosa in Antirrhinum majus. 1992. EMBO. 11: 1239-1249.

59. Hunt, M. C. and Alexson, S. E. 2002. The role Acyl-CoA thioesterases play in mediating intracellular lipid metabolism. Progress in Lipid Research. 41: 99-130.

60. Javelle, M., Vernoud, V., Depège-Fargeix, N., Arnould, C., Oursel, D., Domergue, F., Sarda, X., and Rogowsky, P. M. 2010. Over-expression of the epidermisspecific HD-ZIP IV transcription factor OCL1 in maize identified target genes involved in lipid metabolism and cuticle biosynthesis. Plant Physiology. 154: 273286.

61. Jenks, M.A., Joly, R.J., Peters, P.J., Rich, P.J., Axtell, J.D., and Ashworth, E.A. 1994. Chemically induced cuticle mutation affecting epidermal conductance to water vapor and disease susceptibility in Sorghum bicolor (L.) Moench. Plant Physiology. 105: 1239-1245.

62. Jenks, M. A., Tuttle, H. A., Eigenbrode, S. D., and Feldmann, K. A. 1995. Leaf epicuticular waxes of the Eceriferum mutants in Arabidopsis. Plant Physiology. 108: 369-377.

63. Jones, J. M. and Gould, S. J. 2000. Identification of PTE2, a human peroxisomal long-chain acyl-CoA thioesterase. Biochemical and Biophysical Research Communications. 275: 233-240. 
64. Kannangara, R., Branigan, C., Liu, Y., Penfield, T., Rao, V., Mouille, G., Höfte, H., Pauly, M., Riechmann, J. L., and Broun, P. 2007. The transcription factor WIN1/SHN1 regulates Cutin biosynthesis in Arabidopsis thaliana. Plant Cell. 19:1278-1294.

65. Keller, H., Dryer, C., Medin, J., Mahfoudi, A., Ozato, K., and Wahli, W. 1992. Fatty acids and retinoids control lipid metabolism through activation of peroxisome poliferator-activated receptor-retinoid X receptor heterodimers. Proceedings of the National Academy of Sciences of the United States of America. 90:2160-2164.

66. Kerstiens, G. 1996. Signaling across the divide: a wider perspective of cuticular structure-function relationships. Trends in Plant Science. 1: 125-129.

67. Kiefer, F., Arnold, K., Künzli, M., Bordoli, L., and Schwede, T. 2009. The SWISS-MODEL Repository and associated resources. Nucleic Acids Research. 37: D387-392.

68. Kotaka, M., Kong, R., Wureshi, I., Ho, Q. S., Sun, H., Liew, C. W., Goh, L. P., Cheung, P., Mu, Y., Lescar, J., and Liang, Z-X. 2009. Structure and catalytic mechanism of the thioesterase CalE7 in Enediyne Biosynthesis. The Journal of Biological Chemistry. 284: 15739-15749.

69. Kolattukudy PE. 1987. Lipid-derived defensive polymers and waxes and their role in plant-microbe interaction. In The Biochemistry of Plants, ed. PK Stumpf, EE Conn, 9:291-314. New York: Academic.

70. Krauss P, Markstädter C and Riederer M. 1997. Attenuation of UV radiation by plant cuticles from woody species. Plant, Cell and Environment. 20: 1079-1085. 
71. Kunst, L., and Samuels, A.L. 2003. Biosynthesis and secretion of plant cuticular wax. Progress in Lipid Research. 42: 51-80.

72. Kurata, T., Kawabata-Awai, C., Sakuradani, E., Shimizu, S., Okada, K., and Wada, T. 2003. The YORE-YORE gene regulates multiple aspects of epidermal cell differentiation in Arabidopsis. Plant Journal. 36: 55-66.

73. Lai, C., Kunst, L., and Jetter, R. 2007. Composition of alkyl esters in the cuticular wax on inflorescence stems of Arabidopsis thaliana cer mutants. Plant Journal. 50: 189-196.

74. Larkin, M. A., Blackshields, G., Brown, N. P., Chenna, R., McGettigan, P. A., McWilliams, H., Valentin, F., Wallace, I. M., Wilm, A., Lopez, R., Thompson, J. D., Gibson, T. J., and Higgins, D. G. 2007. ClustalW and ClustalX version 2. Bioinformatics. 23: 2947-2948.

75. Lee, S. B., Jung, S. J., Go, Y. S., Kim, H. U., Cho, H. J., Park, O. K., and Suh, M. C. 2009. The Arabidopsis 3-ketoacyl CoA synthase genes, KCS20 and KCS2/DAISY, are functionally redundant in cuticular wax and root suberin biosynthesis, but differentially controlled by osmotic stress. Plant Journal. 60: $462-475$.

76. Lee, S. B., Go, Y. S., Bae, H. J., Park, J. H., Cho, S. H., Cho, H. J., Lee, D. S., Park, O. K., Hwang, I., and Suh, M. C. 2009. Disruption of glycosylphosphatidylinositol-anchored lipid transfer protein gene altered cuticular lipid composition, increased plastoglobules, and enhanced susceptibility to infection by the fungal pathogen Alternaria brassicicola. Plant Physiology. 150: 42-54. 
77. Leesong, M, Henderson, B. S., Gilling, J. R., Schwab, J. M., and Smith, J. L. 1996. Structure of a dehydratase-isomerase from the bacterial pathway for biosynthesis of unsaturated fatty acids: two catalytic activities in one active site. Structure. 4:253264.

78. Li, F., Wu, X., Lam, P., Bird, D., Zheng, H., Samuels, L., Jetter, R., and Kunst, L. 2008. Identification of the wax ester synthase/acyl-coenzyme A: diacylglycerol acyltransferase WSD1 required for stem wax ester biosynthesis in Arabidopsis. Plant Physiology. 148: 97-107.

79. Li, Y., Beisson, F., Koo, A. J., Molina, I., Pollard, M., and Ohlrogge, J. 2007. Identification of acyltransferases required for cutin biosynthesis and production of cutin with suberin-like monomers. Proceedings of the National Academy of Sciences of the United States of America. 104: 18339-18244.

80. Li, Y.H., Beisson, F., Ohlrogge, J., and Pollard, M. 2007b. Monoacylglycerols are components of root waxes and can be produced in the aerial cuticle by ectopic expression of a suberin-associated acyltransferase. Plant Physiology. 144: 12671277.

81. Li-Beisson, Y., Shorrosh, B., Beisson, F., Andersson, M. X., Arondel, V., Bates, P. D., Baud, S., Bird, D., Debono, A., Durrett, T. P., Franke, R. B., Graham, I. A., Katayama, K., Kelly, A. A., Larson, T., Markham, J. E., Miquel, M., Molina, I., Nishida, I., Rowland, O., Samuels, L., Schmid, K. M., wada, H., Welti, R., Xu, C., Zallot, R., and Ohlrogge, J. 2010. Acyl-Lipid Metabolism. In The Arabidopsis book. American Society of Plant Biologists. 1-65. 
82. Li-Beisson, Y., Pollard, M., Sauveplane, V., Pinot, F., Ohlrogge, J., and Beisson, F. 2010. Nanoridges that characterize the surface morphology of flowers require the synthesis of cutin polyester. Proceedings of the National Academy of Sciences of the United States of America. 106: 22008-22013.

83. Lippold, F., Sanchez, D. H., Musialak, M., Schlereth, A., Scheible, W. R., Hincha, D. K., and Udvardi, M.K. 2009. AtMyb41 regulates transcriptional and metabolic responses to osmotic stress in Arabidopsis. Plant Physiology. 149: 1761-1772.

84. Liu, D. and Post-Beittenmiller, D. 1995. Discovery of an epidermal stearoyl-acyl carrier protein thioesterase. Its potential role in wax biosynthesis. The Journal of Biological Chemistry. 270: 16962-16969.

85. Lopes, M.H., Gil, A.M., Silvestre, A.J.D., and Neto, C.P. 2000. Composition of suberin extracted upon gradual alkaline methanolysis of Quercus suber L. cork. Journal of Agricultural and Food Chemistry. 48: 383-391.

86. Lü, S., Song, T., Kosma, D. K., Parsons, E. P., Rowland, O., and Jenks, M. A. 2009. Arabidopsis CER8 encodes LONG-CHAIN ACYL-COA SYNTHETASE 1 (LACS1) that has overlapping functions with LACS2 in plant wax and cutin synthesis. Plant Journal. 59: 553-564.

87. Lu, J. Y., Verkruyse, L. A., and Hofmann, S. L. 1996. Lipid thioesters derived from acylated proteins accumulate in infantile neuronal ceroid lipofuscinosis: correction of the defect in lymphoblasts by recombinat palmitoyl-protein thioesterase. Proceedings of the National Academy of Sciences of the United States of America. 93: 10046-10050. 
88. Luo, B., Xue, X. Y., Hu, W. L., Wang, L. J., and Chen, X. Y. 2007. An ABC transporter gene of Arabidopsis thaliana, AtWBC11, is involved in cuticle development and prevention of organ fusion. Plant and Cell Physiology. 48: 17901802.

89. Marchler-Bauer, A, Anderson, J. B., Chitsaz, F., Derbyshire, M. K, DeWeese-Scott, C., Fong, J. H., Geer, L. Y., Geer, R. C., Gonzales, N. R., Gwadz, M., He, S., Hurwitz, D. I., Jackson, J. D., Ke, Z., Lanczycki, C. J., Liebert, C. A., Liu, C., Lu, F., Lu, S., Marchler, G. H., Mullokandov, M., Song, J. S., Tasneem, A., Thanki, N., Yamashita, R. A., Zhang, D., Zhang, N., and Bryant, S. H. 2009. CDD: specific function annotation with the Conserved Domain Database. Nucleic Acids Research. 37: 205-210.

90. Markstädter, C., Federle, W., Jeter, R., Riederer, M. and Holldobler, B. 2000. Chemical composition of the slippery epicuticular wax blooms on Maca- ranga (Euphorbiaceae) ant-plants. Chemoecology. 10: 33-40.

91. Martinez, M.A., Zaballa, M.-E., Schaeffer, F., Bellinzoni, M., Albanesi, D., Schujman, G.E., Vila, A.J., Alzari, P. M., and de Mendoza, D. 2010. A novel role of malonyl-ACP in lipid homeostasis. Biochemistry. 49: 3161-3167.

92. Mayer, K. M. and Shanklin, J. 2005. A structural model of the plant acyl-acyl carrier protein thioesterase FatB comprises two helix/4-stranded sheet domains, the $\mathrm{N}$-terminal domain containing residues that affect specificity and the C-terminal domain containing catalytic residues. Journal of Biological Chemistry. 280:36213627. 
93. Mayer, K. M. and Shanklin, J. 2007. Identification of amino acid residues involved in substrate specificity of plant acyl-ACP thioesterases using a bioinformatics-guided approach. BMC Plant Biology. 7: 1.

94. McNevin, J. P., Woodward, W., Hannoufa, A., Feldmann, K. A., and Lemieux, B. 1993. Isolation and characterization of eceriferum (cer) mutants induced by TDNA insertions in Arabidopsis thaliana. Genome. 36: 610-618.

95. Miller, A. A., Clemens, S., Zachgo, S., Giblin, E. M., Taylor, D. C., and Kunst, L. 1999. CUT1, an Arabidopsis gene required for cuticular wax biosynthesis and pollen fertility, encodes a very-long-chain fatty acid condensing enzyme. Plant Cell. 11: 825-838.

96. Miller, A. A., and Kunst, L. 1997. Very-long-chain fatty acid biosynthesis is controlled through the expression and specificity of the condensing enzyme. The Plant Journal. 12: 121-131.

97. Molina, I., Li-Beisson, Y., Beisson, F., Ohlrogge, J. B., and Pollard, M. 2009. Identification of an Arabidopsis feruloyl-coenzyme A transferase required for suberin biosynthesis. Plant Physiology. 151: 1317-1328.

98. Murakami, K., Ide, T., Nakazawa, T., Okazaki, T., Mochizuki, T., and Kadowaki, T. 2001. Fatty-acyl-CoA thioesters inhibit recruitment of steroid receptor coactivator 1 to $\alpha$ and $\gamma$ isoforms of peroxisome-proliferator-activated receptors by competing with agonists. Biochemical Journal. 353: 231-238.

99. Nardini, M. and Dijkstra, B. W. 1999. $\alpha / \beta$ hydrolase fold enzymes: the family keeps growing. Current Opinion in Structural Biology. 9: 732-737. 
100. Neal, A.L., Weinstock, J.O., and Lampen, J.O. 1965. Mechanisms of fatty acid toxicity for yeast. Journal of Bacteriology. 90: 126-131.

101. Negruk, V., Yang, P., Subramanian, M., McNevin, J. P., and Lemieux, B. 1996. Molecular cloning and characterization of the CER2 gene of Arabidopsis thaliana. Plant Journal. 9: 137-145.

102. Ohlrogge, J.B. and Jaworski, J.G. 1997. Regulation of fatty acid synthesis. Annual Review of Plant Physiology and Plant Molecular Biology. 48: 109-136.

103. Ollis, D. L., Cheah, E. Cygler, M., Dijkstra, B., Frolow, F., Franken, S. M., Harel, M., Remington, S. J., Silman, I., Schrag, J., Sussman, J. L., Verschueren, K. H. G., and Goldman, A. 1992. The $\alpha / \beta$ hydrolase fold. Protein Engineering. 5: 197-211.

104. Ossowski, S., Fitz, J., Schwab, R., Riester, M., and Weigel, D. 2005. Personal communication. WMD2-Web MicroRNA Designer. http://wmd2.weigelworld.org.

105. Ossowski , S., Schwab, R., and Weigel, D. 2008. Gene silencing in plants using artificial microRNAs and other small RNAs. The Plant Journal. 53: 674-690.

106. Overath, P., Pauli, G., and Schairer, H. U. 1969. Fatty acid degradation in Escherichia coli. An inducible acyl-CoA synthetase, the mapping of old-mutations, and the isolation of regulatory mutants. European Journal of Biochemistry. 7: 559 $-574$.

107. Panikashvili, D., Savaldi-Goldstein, S., Mandel, T., Yifhar, T., Franke, R. B., Höfer, R., Schreiber, L., Chory, J., and Aharoni, A. 2007. The Arabidopsis DESPERADO/AtWBC11 transporter is required for cutin and wax secretion. Plant Physiology. 145: 1345-1360. 
108. Panikashvili, D., Shi, J. X., Schreiber, L., and Aharoni, A. 2009. The Arabidopsis $D C R$ endoding a soluble BAHD acyltransferase is required for cutin polyester formation and seed hydration properties. Plant Physiology. 151: 1773-1789.

109. Peitsch, M. C. 1995. Protein modeling by E-mail. Bio/Technology. 13: 658-660.

110. Perera, M., Qin, W., Yandeau-Nelson, M., Fan, L., Dixon, P., and Nikolau, B.J. 2010. Biological origins of normal-chain hydrocarbons: a pathway model based on cuticular wax analyses of maize silks. The Plant Journal. 64: 618-632.

111. Pidugu, L. S., Maity, K., Ramaswamy, K., Surolia, N., and Suguna, K. Analysis of proteins with the 'hot dog' fold: prediction of function and identification of catalytic residues of hypothetical proteins. BMC Structural Biology. 9: 37.

112. Piffanelli, P., Ross, J. H. E., and Murphy, D. J. 1998. Biogenesis and function of the lipidic structures of pollen grains. Sexual Plant Reproduction. 11:65-80.

113. Pighin, J. A., Zheng, H., Balakshin, L. J., Goodman, I. P., Western, T. L., Jetter, R., Kunst, L., and Samuels, A. L. 2004. Plant cuticular lipid export requires an ABC transporter. Science. 306: 702-704.

114. Pollard, M., Beisson, F., Li, Y., and Ohlrogge, J. B. 2008. Building lipid barriers: biosynthesis of cutin and suberin. Trends in Plant Science. 13: 236-246.

115. Post-Beittenmiller, D. 1996. Biochemistry and molecular biology of wax production in plants. Annual Review of Plant Physiology and Plant Molecular Biology. 47: 405-430.

116. Raffaele, S., Vailleau, F., Léger, A., Joubès, J., Miersch, O., Huard, C., Blée, E., Mongrand, S., Domergue, F., and Roby, D. 2008. A MYB transcription factor 
regulates very-long-chain fatty acid biosynthesis for activation of the hypersensitive cell death response in Arabidopsis. Plant Cell. 20: 752-567.

117. Rashotte, A. M. Jenks, M. A., and Feldmann, K. A. 2001. Cuticular waxes on eceriferum mutants of Arabidopsis thaliana. Phytochemistry. 57: 115-123.

118. Rashotte, A. M., Jenks, M. A., Ross, A. S., and Feldmann, K. A. 2004. Novel eceriferum mutants in Arabidopsis thaliana. Planta. 219: 5-13.

119. Ray AK, Chen Z, and Stark RE. 1998. Chemical depolymerization studies of the molecular architecture of lime fruit cuticle. Phytochemistry. 49: 65-70.

120. Ray AK and Stark RE. 1998. Isolation and molecular structure of an oligomer produced enzymatically from the cuticle of lime fruit. Phytochemistry. 48: 13131320.

121. Riederer, M. and Schreiber, L. 2001. Protecting against water loss: analysis of the barrier properties of plant cuticles. Journal of Experimental Botany. 52: $2023-$ 2032.

122. Rowland, O., Zheng, H., Hepworth, S. R., Lam, P., Jetter, R., and Kunst, L. 2006. CER4 encodes an alcohol-forming fatty acyl-Coenzyme A reductases involved in cuticular wax production in Arabidopsis. Plant Physiology. 142: 866-877.

123. Rowland, O., Lee, R., Franke, R., Schreiber, L., and Kunst, L. 2007. The cer3 wax biosynthetic gene from Arabidopsis thaliana is allelic to WAX2/YRE/FLP1. FEBS Letters. 581: 3538-3544.

124. Salas, J. J. and Ohlrogge, J. B. 2002. Characterization of substrate specificity of plant FatA and FatB acyl-ACP thioesterases. Archives of Biochemistry and Biophysics. 403: 25-34. 
125. Samach, A., Kohalmi, S. E., Motte, P., Datla, R., and Haughn, G. W. 1997. Divergence of function and regulation of class $\mathrm{B}$ floral organ identity genes. The Plant Cell. 9: 559-570.

126. Samuels, L., Kunst, L., and Jetter, R. 2008. Sealing Plant Surfaces: Cuticular Wax Formation by Epidermal Cells. Annual Review of Plant Biology. 59: 683-707.

127. Schirmer, A., Rude, M. A., Li, X., Popova, E., and del Cardayre, S. B. 2010. Microbial biosynthesis of alkanes. Science. 329: 559-562.

128. Schleberger, C., Sachelaru, P., Brandsch, R., and Schulz, G. E. 2007. Structure and action of a $\mathrm{C}-\mathrm{C}$ bond cleaving alpha/beta-hydrolase involved in nicotine degradation. Journal of Molecular Biology. 367: 409-418.

129. Schmid, M., Davison, T. S., Henz, S. R., Pape, U.J., Demar, M., Vingron, M., Schölkopf, B., Weigel, D., and Lohmann, J. U. 2005. A gene expression map of Arabidopsis thaliana development. Nature Genetics. 37: 501-506.

130. Schneider-Belhaddad and Kolattukudy, P. 2000. Solubilization, Partial Purification, and Characterization of a Fatty Aldehyde Decarbonylase from a Higher Plant, Pisum sativum. Archives of Biochemistry and Biophysics. 377: 341349.

131. Schnurr, J., Shockey, J.M., de Boer, G.-J., and Browse, J. 2002. Fatty acid export from the chloroplast. Molecular characterization of a major plastidial acylcoenzyme A synthetase from Arabidopsis. Plant Physiology. 129: 1700-1709.

132. Schnurr, J., Shockey, J., and Browse, J. 2004. The acyl-CoA synthetase encoded by LACS2 is essential for normal cuticle development in Arabidopsis. Plant Cell. 16: 629-642. 
133. Schujman, G. E., Guerin, M., Buschiazzo, A., Schaeffer, F., Llarrull, L. 1., Reh, G., Vila, A. J., Pedro, M. A., and de Mendoza, D. 2006. Structural basis of lipid biosynthesis regulation in gram-positive bacteria. The EMBO Journal. 25: 40744083.

134. Shockey, J. M., Fulda, M. S., and Browse, J. A. 2002. Arabidopsis contains nine long-chain acyl-coenzyme A synthetase genes that participate in fatty acid and glycerolipid metabolism. Plant Physiology. 129: 1710-1722.

135. Sieber, P., Schorderet, M., Ryser, U., Buchala, A., Kolattukudy, P., Metraux, J.-P., and Nawrath, C. 2000. Transgenic Arabidopsis plants expressing a fungal cutinase show alterations in the structure and properties of the cuticle and postgenital organ fusions. Plant Cell. 12: 721-738.

136. Somerville, S. C. and Ogren, W. L. 1982. Isolation of photorespiratory mutants of Arabidopsis. In RB Hallick, NH Chua, eds, Methods in Chloroplast Molecular Biology. Elsevier, New York, 129-139.

137. Suh, M. C., Samuels, L., Jetter, R., Kunst, L., Pollard, M., Ohlrogge, J., and Beisson, F. 2005. Cuticular lipid composition, surface structure, and gene expression in Arabidopsis stem epidermis. Plant Physiology. 139: 1649-1665

138. Thoden, J. B., Zhuang, Z., Dunaway-Mariano, D., and Holden, H.M. 2003. The structure of 4-hydroxybenzoyl-CoA thioesterase from Arthrobacter sp. strain SU. Journal of Biological Chemistry. 278: 43709-43716.

139. Tilton, G.B., Shockey, J.M., and Browse, J. 2004. Biochemical and molecular characterization of $\mathrm{ACH} 2$, an acyl-CoA thioesterase from Arabidopsis thaliana. The Journal of Biological Chemistry. 279: 7487-7494. 
140. Todd, J., Post-Beittenmiller, D., and Jaworski, J. G. 1999. KCS1 encodes a fatty acid elongase 3-ketoacyl-CoA synthase affecting wax biosynthesis in Arabidopsis thaliana. Plant Journal. 17: 119-130.

141. Toufighi, K., Brady, S. M., Autsin, R., Ly, E., and Provart, N. J. 2005. The botany array resource: e-Northerns, expression angling and promoter analyses. The Plant Journal. 43: 153-163.

142. Vioque, J. and Kolattukudy, P. E. 1997. Resolution and purification of an aldehyde-generating and an alcohol-generating fatty acyl-coA reductase from Pea Leaves (Pisum sativum L.). Archives of biochemistry and biophysics. 340: 64-72.

143. von Wettstein-Knowles P. 1982. Elongase and epicuticular wax biosynthesis. Physiologie Végétale. 20: 797-809.

144. Wang, X. and Kolattukudy, P. E. 1995. Solubilization and purification of aldehydegenerating fatty acyl-CoA reductase from geen alga Botryococcus braunii. FEBS Letters. 370: 15-18.

145. Wei, J., Kang, H., and Cohen, D. 2009. Thioesterase superfamily member 2 (Them2)/acyl-CoA thioesterase 13 (Acot13): a homotetrameric hotdog fold thioesterase with selectivity for long-chain fatty acyl-CoAs. Biochemical Journal. 421: 311-322.

146. Wellesen, K., Durst, F., Pinot, F., Benveniste, I., Nettesheim, K., Wisman, E., Steiner-Lange, S., Saedler, H., and Yephremov, A. 2001. Functional analysis of the LACERATA gene of Arabidopsis provides evidence for different roles of fatty acid omega-hydroxylation in development. Proceedings of the National Academy of Sciences of the United States of America. 98: 9694-9699. 
147. Weng, H., Molina, I., Schockey, J., and Browse, J. 2010. Organ fusion and defective cuticle function in a lacs1lacs2 double mutant of Arabidopsis. Planta. 231: $1089-1100$.

148. Winter, D., Vinegar, B., Nahal, H., Ammar, R., Wilson, G.V. and Provart, N. J. 2007. An "Electronic Fluorescent Pictograph" browser for exploring and analyzing large-scale biological data sets. PLOS ONE. 2: e718.

149. Wu, J.T., Chiang, Y.R., Huang, W.Y., and Jan, W.N. 2006. Cytotoxic effects of free fatty acids on phytoplankton algae and cyanobacteria. Aquatic Toxicology. 80 : 338-345.

150. Xia Y., Nikolau, B. J., and Schnable, P. S. 1996. Cloning and characterization of CER2, an Arabidopsis gene that affects cuticular wax accumulation. Plant Cell. 8: 1291-1304.

151. Xia Y., Nikolau, B. J., and Schnable, P. S. 1997. Developmental and hormonal regulation of the Arabidopsis CER2 gene that codes for a nuclear-localized protein required for the normal accumulation of cuticular waxes. Plant Physiology. 115: $925-937$.

152. Xiao, F., Goodwin, S. M., Xiao, Y., Sun, Z., Baker, D., Tang, X., Jenks, M. A., and Zhou, J-M. 2004. Arabidopsis CYB86A2 represses Pseudomonas syringae type III genes and is required for cuticle development. The EMBO Journal. 23: 2903-2913.

153. Yu, G., Nguyen, T.T.H, Guo, Y., Schauvinhold, I., Auldridge, M.E., Bhuiyn, N., Ben-Israel, I., Iijima, Y., Fridman, E., Noel, J. P., and Pichersky, E. 2010. Enzymatic functions of wild tomato methylketone synthases 1 and 2. Plant Physiology. 154: 67-77. 
154. Zhang, X., Henriques, R., Lin, S., Niu, Q., and Chua, N. 2006. Arabidopsismediated transformation of Arabidopsis thaliana using the floral dip method. Nature Protocols. 1: 641-646.

155. Zhuang, Z., Gartemann, K-H., Eichenlaub, R., and Dunaway-Mariano, D. 2003. Characterization of the 4-hydrozybenzoyl-coenzyme A thioesterase from Arthrobacter sp. Strain SU. Applied and Environmental Microbiology. 69: 27072711.

156. Zhuang, Z., Song, F., Zhao, H., Li, L., Cao, J., Eisenstein, E., Herzberg, O., and Dunaway-Mariano, D. 2008. Divergence of function in the hot dog fold enzyme superfamily: the bacterial thioesterase YciA. Biochemistry. 47: 2789-2796. 